\title{
Three-particle systems with resonant subprocesses in a finite volume
}

\author{
Raúl A. Briceño, ${ }^{1,2 *}$ Maxwell T. Hansen, ${ }^{3, \dagger}$ and Stephen R. Sharpe ${ }^{4, \$}$ \\ ${ }^{1}$ Thomas Jefferson National Accelerator Facility, \\ 12000 Jefferson Avenue, Newport News, Virginia 23606, USA \\ ${ }^{2}$ Department of Physics, Old Dominion University, Norfolk, Virginia 23529, USA \\ ${ }^{3}$ Theoretical Physics Department, CERN, 1211 Geneva 23, Switzerland \\ ${ }^{4}$ Physics Department, University of Washington, Seattle, Washington 98195-1560, USA
}

(Received 29 October 2018; published 30 January 2019)

\begin{abstract}
In previous work, we have developed a relativistic, model-independent three-particle quantization condition, but only under the assumption that no poles are present in the two-particle K matrices that appear as scattering subprocesses [M. T. Hansen and S. R. Sharpe, Phys. Rev. D 90, 116003 (2014); M. T. Hansen and S. R. Sharpe, Phys. Rev. D 92, 114509 (2015); R. A. Briceño et al., Phys. Rev. D 95, 074510 (2017).]. Here we lift this restriction, by deriving the quantization condition for identical scalar particles with a G-parity symmetry, in the case that the two-particle $\mathrm{K}$ matrix has a pole in the kinematic regime of interest. As in earlier work, our result involves intermediate infinite-volume quantities with no direct physical interpretation, and we show how these are related to the physical three-to-three scattering amplitude by integral equations. This work opens the door to study processes such as $a_{2} \rightarrow \rho \pi \rightarrow \pi \pi \pi$, in which the $\rho$ is rigorously treated as a resonance state.
\end{abstract}

DOI: 10.1103/PhysRevD.99.014516

\section{INTRODUCTION}

Studies of hadronic resonances using lattice QCD (LQCD) have progressed rapidly in recent years. ${ }^{1}$ The present frontier of this effort involves resonances that have significant branching ratios into channels with three (or more) particles. Here the results from lattice calculations are, in some cases, more advanced than the theoretical developments needed to interpret them. In particular, energy levels above three-particle thresholds are already being calculated, using three-particle operators [4]. Thus a fully developed theoretical formalism to interpret LQCD quantities in this sector is of great importance. In recent years significant progress has been made, using a variety of approaches [5-17]. In this work we consider the relativistic model-independent framework of Refs. [5-7], and remove the last major theoretical restriction on this formalism.

\footnotetext{
*rbriceno@jlab.org

†maxwell.hansen@cern.ch

*srsharpe@uw.edu

${ }^{1}$ For recent reviews, see Refs. [1-3].
}

Published by the American Physical Society under the terms of the Creative Commons Attribution 4.0 International license. Further distribution of this work must maintain attribution to the author(s) and the published article's title, journal citation, and DOI. Funded by SCOAP ${ }^{3}$.
LQCD studies of resonances proceed in two basic steps. First, one uses numerical LQCD to determine the energy levels in a finite volume for a given range of total energy. Second, these levels are related to infinite-volume scattering parameters by solving a quantization condition. ${ }^{2}$ In the case of a single channel of identical scalar particles, the relation between finite-volume energies and the scattering amplitude was first derived by Lüscher $[18,19]$. This has since been extended to describe all possible, multichannel two-particle systems [20-25] and by now there is a large body of work extracting energy levels above multiple open thresholds and relating these to the different components of the coupled-channel scattering amplitudes [26-31]. For resonances with threeparticle decay channels, a further step is required, in which intermediate infinite-volume quantities are related to the scattering amplitudes. This step also requires knowledge of the scattering amplitudes in each of the two-particle subsystems.

The approach we follow here was originally derived in Refs. [5,6] under two major assumptions: first, that a G-paritylike symmetry forbids $2 \leftrightarrow 3$ transitions and, second, that two-particle subsystems are nonresonant

\footnotetext{
${ }^{2}$ In practice, this requires truncation of the quantization condition by assuming that higher partial waves are negligible. Such truncation schemes for the three-particle case have been discussed in Refs. [5,7,11,12,14-16]. We do not consider these further in the present work.
} 
within the kinematic range of interest (or, more precisely, that the two-particle $\mathrm{K}$ matrices have no poles). ${ }^{3}$ We removed the former restriction in Ref. [7], and it is the purpose of the present paper to lift the second restriction, i.e., to allow arbitrary interactions in the twoparticle subsystems. This removes the last major theoretical obstacle to general implementation of the formalism.

Removing the restriction on subchannel $\mathrm{K}$ matrices is necessary for the application of the formalism to many interesting three-particle systems. Consider, for example, the three-pion system in the isospin-symmetric limit. Only for the maximal isospin channel, $I=3$, are all two-pion subchannels nonresonant (since they all have $I_{\text {sub }}=2$ ). For $I_{\text {tot }}<3$, however, the subchannels can have $I_{\text {sub }}=1$ or 0 , and thus contain either the $\rho$ or $\sigma$ resonance, respectively. For example, the $a_{2}(1320)\left(I^{G}=1^{-}, J^{\mathrm{PC}}=2^{++}\right)$decays predominantly to three pions with the $\rho$ and $f_{2}(1270)$ resonances in two-pion subchannels. Another example where subchannel resonances must be included is the Roper resonance, which has a significant branch to the $\Delta \pi \rightarrow p \pi \pi$ channel.

It is useful to recall the reason why the analysis in Refs. [5,7] had to assume the absence of poles in the twoparticle $\mathrm{K}$ matrix, $\mathcal{K}_{2}$. These works study finite-volume correlation functions and determine the spectrum from the position of the poles in these functions. The correlation functions are considered to all orders in perturbation theory in an arbitrary effective field theory. The core step in the analysis is the replacement of the 3-momentum sums that appear in finite volume with the corresponding infinitevolume integrals, together with a volume-dependent residue. If the summand is smooth, this residue is exponentially suppressed (i.e., suppressed by $e^{-m L}$ where $m$ is the particle mass and $L$ the box size), and such exponentially suppressed corrections are assumed negligible. However, if the summand is singular then the residue falls only like inverse powers of $L$, and must be kept. Such singularities occur either when intermediate states can go on shell or when intermediate infinite-volume quantities are themselves singular. In our approach the latter class of singularities arises as K-matrix poles. In Refs. [5,7] we did not include the finite-volume effects associated with these and thus the formalism derived in those works only applies if they are absent.

Poles in $\mathcal{K}_{2}$ do not correspond to physical particles. If the theory has a narrow resonance in the two-particle subsystem the two-body scattering amplitude, $\mathcal{M}_{2}$, will have a complex-valued pole close to the real axis. For this scenario, $\mathcal{K}_{2}$ will have a real-valued pole close to that

\footnotetext{
${ }^{3}$ In addition the particles were taken to be identical and spinless. Based on experience with the two-particle case, we expect the extensions to multiple channels of nonidentical and nondegenerate particles, as well as particles with intrinsic spin, will be relatively straightforward.
}

of $\mathcal{M}_{2}$. Therefore, the $\mathcal{K}_{2}$ pole is approximately equal to the mass of the resonance. Away from the very-narrow limit of a resonance, however, $\mathcal{K}_{2}$ poles do not have a direct physical interpretation. Nevertheless, at intermediate stages of the analysis of Ref. [5], terms appear whose summands contain such singularities. These lead to additional powerlaw finite-volume dependence, and this must be accounted for, as it ultimately impacts the form of the quantization condition. The analysis presented here incorporates all such contributions.

It is worth noting that one may envision taking a different approach than that proposed in Refs. [5,7] in which twoparticle subprocesses are encoded via $\mathcal{M}_{2}$ instead of $\mathcal{K}_{2}$, and thus the scattering amplitude appears inside of the summand. The original reason for preferring $\mathcal{K}_{2}$ is that $\mathcal{M}_{2}$ has a cusp at the two-particle threshold and one must then include finite-volume effects associated with this singularity. Furthermore, if the system contains a narrow resonance, of width $\Gamma$, then $\mathcal{M}_{2}$ varies rapidly as a function of energy and this induces neglected $e^{-\Gamma L}$ volume effects, if the contribution is not explicitly incorporated. In addition, in QCD many resonances lie close to thresholds, leading to dynamically enhanced cusp effects. In short, one would have to develop a framework to address finite-volume effects associated with all possible scenarios. With these considerations in mind, we find it preferable to work with $\mathcal{K}_{2}$ and properly treat its poles in the kinematic window of interest.

In order to keep track of these singularities, we find it convenient to express the problem in terms of two effective channels: one containing the physical three-particle state, and a second built from a particle and a pseudoparticle arising from the $\mathcal{K}_{2}$ pole, which we refer to as the " $\rho \pi$ channel." The quantization condition turns out to take a relatively simple form in this presentation, one that is similar to that in the multichannel two-particle problem [22-24]. An important consistency check is that our final expression for the finite-volume correlator does not contain $\rho \pi$ poles, despite their appearance at intermediate stages.

The addition of an unphysical channel appears at first as a negative feature of our approach. We have explored various alternatives that do not require this artifact, but have not yet been able to use them to derive a useful alternative formalism. There is, however, one reason to view the appearance of this channel as natural. To explain this, we return to the example of the $\rho$ resonance, and imagine continuously increasing the quark masses, starting from their physical values. As is well known, as this is done, the $\rho$ becomes narrower, eventually becoming a bound state at threshold, and, beyond that, a physical particle lying below the two-pion threshold. If the masses are chosen such that the $\rho$ is deeply bound, then the pole in $\mathcal{K}_{2}$ moves far below threshold and becomes irrelevant to our formalism. Thus, in this case, the unphysical channel is no longer needed. However, the presence of the $\rho$ particle implies that one should use the $2+3$ particle formalism of Ref. [7], 
including a physical $\rho \pi$ channel. Given that the stable $\rho$ can be described in terms of a new open channel, it is natural that this is continuously connected to the effective two-particle channel for narrow resonances that arises in this work.

As was the case in Refs. [5-7], the derivation of the quantization condition is rather lengthy, despite the fact that we have found ways to shorten and simplify certain steps compared to the earlier works. To make this paper more accessible, we have focused in the main text on the logic and key steps of the derivation, pushing most of the details into Appendices. In addition, we have provided a Mathematica notebook as Supplemental Material [32] in which the package The NCAlgebra Suite is used to check the key results by algebraically manipulating matrices of unspecified size as generic noncommuting objects [33].

This article is organized as follows. We begin in Sec. II by presenting the final result and defining all of the objects appearing in it. This section is meant to stand alone so that the lattice practitioner does not need to look elsewhere in order to make use of the result. In Sec. III we present the derivation of the quantization condition, with technical details given in Appendix B. The quantization condition is written in terms of the three-body $\mathrm{K}$ matrix, which we relate to the physical scattering amplitude in Sec. IV. We summarize, compare to previous work, and give an outlook in Sec. V.

The framework presented here relies heavily on two facts: First, that the off-shell version of $\mathcal{K}_{2}$ has the same poles as its on-shell limit and, second, that at the residues of the poles of the off-shell $\mathcal{K}_{2}$ can be written as a product of functions separately describing the incoming and outgoing two-particle states. In Appendix A we demonstrate these two results using constraints from unitarity and all-orders perturbation theory.

\section{SUMMARY OF THE FINAL RESULT}

The main result of this article is a quantization condition with solutions equal to the energies of finite-volume threeparticle states in a generic, relativistic quantum field theory. In contrast to earlier work, this result also holds for systems with a two-particle resonant subchannel. The particles are assumed to be identical, of physical mass $m$, and to have a G-paritylike symmetry that restricts interactions to those involving an even number of fields.

We assume that $\mathcal{K}_{2}$ diverges only for a single angular momentum, denoted $J$, in the energy range of interest, specified below. We further assume that there is only one pole in $\mathcal{K}_{2}^{(J)}$ in this energy range, occurring when the two-particle center of mass (c.m.) energy equals $M^{4}$ These assumptions simplify the discussion and derivation.

\footnotetext{
${ }^{4}$ In the following we refer to this energy as the "resonance mass," which is a convenient label despite the fact that the correct definition of the resonance mass differs for all but a very narrow resonance. As noted in the introduction, we also refer to the resonance channel as the $\rho$.
}

The extension to completely general $\mathrm{K}$ matrices, achieved by promoting certain quantities introduced here to matrices, will be described in a future publication.

The result presented in this work holds for fields restricted to a cubic spatial volume of side length $L$, with periodic boundary conditions. Following the pattern that is by now well established from previous work [5-7], we find that for a given total momentum, $\vec{P}=2 \pi \vec{n}_{P} / L$, the discrete finite-volume spectrum is given by all solutions in $E$ to the condition

$$
\operatorname{det}\left[1+\mathcal{K}_{\mathrm{df}}\left(E^{*}\right) \mathcal{F}(E, \vec{P}, L)\right]=0,
$$

where $E^{*}=\sqrt{E^{2}-\vec{P}^{2}}$ is the total energy in the c.m. frame. Here both $\mathcal{K}_{\mathrm{df}}\left(E^{*}\right)$ and $\mathcal{F}(E, \vec{P}, L)$ are matrices on a twochannel space

$$
\begin{aligned}
i \mathcal{K}_{\mathrm{df}} & \equiv\left(\begin{array}{ll}
i \mathcal{K}_{\mathrm{df}, \tilde{2} \tilde{2}} & i \mathcal{K}_{\mathrm{df}, \tilde{2} 3} \\
i \mathcal{K}_{\mathrm{df}, 3 \tilde{2}} & i \mathcal{K}_{\mathrm{df}, 33}
\end{array}\right), \\
i \mathcal{F} & \equiv\left(\begin{array}{ll}
i F_{\tilde{2} \tilde{2}} & i F_{\tilde{2} 3} \\
i F_{3 \tilde{2}} & i F_{33}
\end{array}\right),
\end{aligned}
$$

where the index 3 denotes the three-particle channel while $\tilde{2}$ denotes an effective two-particle channel containing the two-particle resonance with the third nonresonating particle. This result holds up to neglected corrections of the form $e^{-m L}$, with $m$ being the physical mass of the stable particle, and applies only in the region $m<E^{*}<5 m$.

In the remainder of this section we provide the definitions of the quantities $\mathcal{K}_{\mathrm{df}}$ and $\mathcal{F}$ appearing in the quantization condition. We only note here that $\mathcal{K}_{\mathrm{df}}$ is a real, infinite-volume quantity that is related to the three-tothree scattering amplitude, while $\mathcal{F}$ has volume dependence but can be expressed in terms of known geometric functions together with the two-particle scattering amplitude, including parameters describing the K-matrix pole.

We discuss strategies for the practical implementation of the quantization condition, the generalization to multiple $\mathrm{K}$-matrix poles, and the relation of this result to earlier work, in Sec. V.

\section{A. Kinematics}

In this subsection we introduce the kinematic variables used throughout the paper to describe two- and threeparticle states, and the index space implicit in the matrices appearing in the quantization condition, Eq. (1). These results are summarized in Table I, which we hope provides a useful reference for the reader. Many of the results are self-explanatory; for the others we provide further explanation in the following.

Each entry in the two-by-two matrices $\mathcal{K}_{\mathrm{df}}$ and $\mathcal{F}$ is itself a matrix in a space that describes the on-shell degrees of 
TABLE I. Summary of kinematics used throughout the paper.

\begin{tabular}{|c|c|c|}
\hline Quantity & Definition/key relation & Description \\
\hline \multicolumn{3}{|c|}{ Basic kinematics used throughout } \\
\hline$\vec{k}$ & $\left(k_{x}, k_{y}, k_{z}\right)=2 \pi \vec{n} / L$ & 3-momentum (often of the spectator particle) \\
\hline$\omega_{k}$ & $\sqrt{\vec{k}^{2}+m^{2}}$ & On-shell time component of 4-vector $k^{\mu}$ (with physical mass $m$ ) \\
\hline$\ell m$ & Indices on $Y_{\ell m}$ & Angular-momentum indices (e.g., of the nonspectator pair) \\
\hline$M_{J}$ & $M_{J}=-J,-J+1, \ldots, J$ & Azimuthal component of total angular momentum $J$ \\
\hline \multicolumn{3}{|c|}{ Multiparticle energies and momenta } \\
\hline$(E, \vec{P})$ & $\vec{P}=2 \pi \vec{n}_{P} / L$ & Total energy and momentum of the three-particle state \\
\hline$P_{2, k}$ & $\left(E-\omega_{k}, \vec{P}-\vec{k}\right)$ & 4-momentum of the nonspectator pair or of the resonance \\
\hline$E_{2, k}^{*}$ & $\sqrt{\left(E-\omega_{k}\right)^{2}-(\vec{P}-\vec{k})^{2}}$ & Energy of the nonspectator pair (two-particle c.m. frame) \\
\hline$q_{2, k}^{*}$ & $\sqrt{E_{2, k}^{* 2} / 4-m^{2}}$ & On-shell momentum of a nonspectator (two-particle c.m. frame) \\
\hline \multicolumn{3}{|c|}{ Individual particles within the three-particle state } \\
\hline$\vec{a}, \vec{b}_{k a}$ & $\vec{b}_{k a} \equiv \vec{P}-\vec{k}-\vec{a}$ & Individual 3-momenta of the nonspectators (finite-volume frame) \\
\hline$\left(\omega_{a}, \vec{a}\right)$ & & 4-momentum of the $a$-momentum particle (finite-volume frame) \\
\hline$\left(\omega_{a ; 2, k}^{*}, \vec{a}_{2, k}^{*}\right)$ & & 4-momentum of the $a$-momentum particle (two-particle c.m. frame) \\
\hline$\left(E-\omega_{k}-\omega_{a}, \vec{b}_{k a}\right)$ & & 4-momentum of the $b$-momentum particle (finite-volume frame) \\
\hline$\left(E_{2, k}^{*}-\omega_{a ; 2, k}^{*}, \vec{b}_{k a ; 2, k}^{*}\right)$ & $\vec{b}_{k a ; 2, k}^{*}=-\vec{a}_{2, k}^{*}$ & 4-momentum of the $b$-momentum particle (two-particle c.m. frame) \\
\hline$\omega_{P k a}$ & $\sqrt{m^{2}+(\vec{P}-\vec{k}-\vec{a})^{2}}$ & On-shell time component of the $b$-momentum particle \\
\hline
\end{tabular}

Individual particles within the $\tilde{2}$-state

$M \quad \lim _{E_{2}^{*} \rightarrow M} \mathcal{K}_{2}\left(E_{2}^{*}\right)=\infty \quad$ Position of the $\mathcal{K}_{2}$ pole

$q_{\rho}^{*}$ defined via $E^{*}=\sqrt{m^{2}+q_{\rho}^{* 2}}+\sqrt{M^{2}+q_{\rho}^{* 2}} \quad$ On-shell momentum of the $\tilde{2}$ spectator (c.m. frame)

$\omega_{\rho, k} \quad \sqrt{M^{2}+(\vec{P}-\vec{k})^{2}} \quad$ On-shell time component of the resonance

$\left(\omega_{k}, \vec{k}\right)$

4-momentum of the $\tilde{2}$ spectator (finite-volume frame)

$\left(E-\omega_{k}, \vec{P}-\vec{k}\right)$

4-momentum of the resonance (finite-volume frame)

$\left(\omega_{k}^{*}, \vec{k}^{*}\right)$

4-momentum of the $\tilde{2}$ spectator (c.m. frame)

$\left(E^{*}-\omega_{k}^{*},-\vec{k}^{*}\right)$

4-momentum of the resonance (c.m. frame)

On-shell conditions and index spaces

$E-\omega_{k}-\omega_{a}=\omega_{P k a} \Leftrightarrow E_{2, k}^{*}=2 \omega_{a ; 2, k}^{*} \Leftrightarrow a_{2, k}^{*}=q_{2, k}^{*}$

Equivalent on-shell conditions for the 3-particle state

$E-\omega_{k}=\omega_{\rho, k} \Leftrightarrow E^{*}-\omega_{k}^{*}=\sqrt{M^{2}+k^{* 2}} \Leftrightarrow k^{*}=q_{\rho}^{*}$

Equivalent on-shell conditions for the $\tilde{2}$-state

$k \ell m=\vec{k}, \ell, m=k_{x}, k_{y}, k_{z}, \ell, m$ where $k_{i}=2 \pi n_{i} / L$

Index space for an on-shell 3-state (implicit with 3 subscript)

$M_{J}, \ell, m$

Index space for an on-shell $\tilde{2}$-state (implicit with $\tilde{2}$ subscript)

freedom, either for three particles or for the resonance together with the spectator. In particular, 3, when used as an index, is shorthand for $3 ; k \ell m=3 ; k_{x} k_{y} k_{z} \ell m$ and $\tilde{2}$ is shorthand for $\tilde{2} ; M_{J} \ell m$. We use $\tilde{2}$ rather than 2 to emphasize that the K-matrix pole does not correspond to a physical particle, and so the $\tilde{2}$ channel is not a physical two-particle channel.

In the three-particle state, one of the three particles, referred to as the spectator, carries the 3-momentum $\vec{k}=\left(k_{x}, k_{y}, k_{z}\right)$. In infinite volume this momentum can take on a continuous range of values (within the range allowed by total energy and momentum conservation), but in our quantization condition it is restricted to discrete values: $\vec{k}=2 \pi \vec{n} / L$ where $\vec{n}$ is a 3 -vector of integers. Within the three-particle state, $\ell m$ describes the angular momentum of the nonspectator pair.

In the $\tilde{2}$ state, $M_{J}$ labels the different azimuthal components for a K-matrix pole with angular momentum $J$. Roughly speaking, it plays the role of a channel index, labeling different degrees of freedom rather than 
different momentum configurations. For a given value of $M_{J}, \ell m$ describes the angular momentum of the spectator-resonance pair. ${ }^{5}$

The kinematics used for the on-shell three-particle state are described in detail in Refs. $[5,6]$. For completeness, and to introduce new notation, we summarize the discussion here. For a given total energy and momentum, $(E, \vec{P})$, we label one of the three particles (the spectator) with on-shell 4-momentum $\left(\omega_{k}, \vec{k}\right)$, where $\omega_{k}=\sqrt{\vec{k}^{2}+m^{2}}$. The 4-momentum of the remaining two particles is then $P_{2, k} \equiv$ $\left(E-\omega_{k}, \vec{P}-\vec{k}\right)$ and their two-particle c.m. energy is

$$
E_{2, k}^{*}=\sqrt{P_{2, k}^{2}}=\sqrt{\left(E-\omega_{k}\right)^{2}-(\vec{P}-\vec{k})^{2}} .
$$

We denote the individual 3-momenta of these two particles in the finite-volume frame by $\vec{a}$ and $\vec{b}_{k a}=\vec{P}-\vec{k}-\vec{a}$.

Often we must consider the case were the $\vec{a}$ particle is on shell with 4-momentum $\left(\omega_{a}, \vec{a}\right)$ whereas the $\vec{b}$ particle is not necessarily on shell, and carries 4-momentum $\left(E-\omega_{k}-\omega_{a}, \vec{b}_{k a}\right)$. Boosting the 4-vectors corresponding to $\vec{a}$ and $\vec{b}$ to the two-particle c.m. frame then gives, respectively,

$$
\begin{aligned}
& \left(\omega_{a ; 2, k}^{*}, \vec{a}_{2, k}^{*}\right), \\
& \left(E_{2, k}^{*}-\omega_{a ; 2, k}^{*}, \vec{b}_{k a ; 2, k}^{*}\right)=\left(E_{2, k}^{*}-\omega_{a ; 2, k}^{*},-\vec{a}_{2, k}^{*}\right) .
\end{aligned}
$$

Here the notation is somewhat involved as we must label both the momenta and the frame. Finally, we need to know the conditions on the kinematic variables such that the $\vec{b}$ particle is also on shell; these are given towards the bottom of Table I. The upshot is that, for three on-shell particles with total energy and momentum $(E, \vec{P})$, the remaining degrees of freedom are the spectator momentum, $\vec{k}$, and the direction of the $\vec{a}$ particle in the nonspectator-pair c.m. frame, $\hat{a}_{2, k}^{*}$. Decomposing the latter in spherical harmonics leads to the indices $\vec{k}, \ell, m$, which we abbreviate to $k \ell m{ }^{6}$

We turn now to the $\tilde{2}$ state, built from a particle of mass $m$ and the resonance of mass $M$. In the overall c.m. frame, each of these has a 3-momentum with a magnitude that we denote by $q_{\rho}^{*}$, given by solving

$$
E^{*}=\sqrt{m^{2}+q_{\rho}^{* 2}}+\sqrt{M^{2}+q_{\rho}^{* 2}} .
$$

In the finite-volume frame, if the particle has momentum $\vec{k}$ and is on shell, then the resonance has 4-momentum

\footnotetext{
${ }^{5}$ We stress that the index pair $\ell m$ plays a very different role in the 3 and $\tilde{2}$ states. This causes no problems, however, as these two sets of indices are never contracted.

${ }^{6}$ As mentioned above, the quantization condition depends only on the allowed finite-volume spectator momenta, $\vec{k}=2 \pi \vec{n} / L$ with $\vec{n}$ being a 3 -vector of integers.
}

$\left(E-\omega_{k}, \vec{P}-\vec{k}\right)$. Boosting these to the overall c.m. frame gives $\left(\omega_{k}^{*}, \vec{k}^{*}\right)$ and $\left(E^{*}-\omega_{k}^{*},-\vec{k}^{*}\right)$. The second particle is then on-shell when any of the three equivalent conditions listed in Table I are satisfied.

Thus, for fixed $(E, \vec{P})$, the two on-shell particles have remaining degree of freedom $\hat{k}^{*}$ and decomposing this in spherical harmonics gives the indices $\ell, m$. Combining this with the azimuthal angular momentum of the resonance gives the full index set, $M_{J} \ell m$.

\section{B. K-matrix poles}

The central aim of this paper is to include the finitevolume effects from poles in $\mathcal{K}_{2}$. In order to complete the definitions of the quantities entering the quantization condition, we need to understand the properties of these poles. This is nontrivial, because, unlike poles in the scattering amplitude, poles in $\mathcal{K}_{2}$ do not correspond to propagation of physical degrees of freedom. Nevertheless, as we show in this subsection and the accompanying Appendix A, two key results do carry over from poles in $\mathcal{M}_{2}$ : the off-shell $\mathrm{K}$ matrix has the same poles as the onshell version, and the residues of the poles factorize. Both results play an important role in the subsequent derivation.

We begin by recalling that the $\ell$ th angular-momentum component of the two-to-two on-shell scattering amplitude satisfies a unitarity constraint, relating it to the scattering phase shift via

$$
\mathcal{M}_{2}^{(\ell)}\left(P_{2, k}^{2}\right)=\frac{16 \pi E_{2, k}^{*}}{q_{2, k}^{*}} \frac{1}{\cot \delta_{\ell}\left(q_{2, k}^{*}\right)-i},
$$

or equivalently

$\mathcal{M}_{2}^{(\ell)}\left(P_{2, k}^{2}\right)^{-1}-\left[\frac{16 \pi E_{2, k}^{*}}{q_{2, k}^{*} \cot \delta_{\ell}\left(q_{2, k}^{*}\right)}\right]^{-1}=-i \frac{q_{2, k}^{*}}{16 \pi E_{2, k}^{*}}$.

In anticipation of three-particle scattering, we have taken the squared c.m. energy in the two-to-two amplitude as $P_{2, k}^{2}=E_{2, k}^{* 2}$, where we recall that $P_{2, k} \equiv\left(E-\omega_{k}, \vec{P}-\vec{k}\right)$ is our notation for the 4-momentum of the nonspectator pair (see Table I). Thus the spectator momentum $\vec{k}$ serves a proxy for the two-particle c.m frame energy. We are assuming in Eqs. (7) and (8) that the scattering is above threshold and in the region where only two-particle states can propagate, $2 m \leq E_{2, k}^{*}<4 m$.

The quantity appearing in square braces in Eq. (8) defines the conventional $\mathrm{K}$ matrix when working above threshold. It is a real function containing all dynamical information about the two-particle scattering. We also need the continuation below threshold, and here, following Ref. [5], we use a nonstandard choice that is convenient for the derivation of the quantization condition. Our K matrix is given by 


$$
\mathcal{M}_{2}^{(\ell)}\left(P_{2, k}^{2}\right)^{-1}-\mathcal{K}_{2}^{(\ell)}\left(P_{2, k}^{2}\right)^{-1} \equiv H(\vec{k}) \tilde{\rho}\left(P_{2, k}^{2}\right),
$$

where $\tilde{\rho}$ is the standard phase-space factor, including below-threshold analytic continuation,

$$
\tilde{\rho}\left(P_{2, k}^{2}\right) \equiv \frac{1}{16 \pi \sqrt{P_{2, k}^{2}}} \times \begin{cases}-i \sqrt{P_{2, k}^{2} / 4-m^{2}}, & (2 m)^{2}<P_{2, k}^{2}, \\ \left|\sqrt{P_{2, k}^{2} / 4-m^{2}}\right|, & 0<P_{2, k}^{2} \leq(2 m)^{2},\end{cases}
$$

and $H(\vec{k})$ is a smooth, real cutoff function that equals 1 when $E_{2, k}^{*} \geq 2 m$ (so that the particles in the nonspectator pair can propagate on-shell) and then smoothly interpolates to 0 in the subthreshold region. Our choice of $\mathcal{K}_{2}$ differs from the analytic continuation of the above-threshold $\mathrm{K}$ matrix once $H$ differs from unity.

Although we do not need to make a choice of $H$ for the derivation, it is useful to have one in mind as an example. The choice suggested in Ref. [5], and used in our recent numerical investigation [16], is

$$
H(\vec{k}) \equiv J\left(P_{2, k}^{2} /\left[4 m^{2}\right]\right),
$$

with

$$
J(x) \equiv \begin{cases}0, & x \leq 0 \\ \exp \left(-\frac{1}{x} \exp \left[-\frac{1}{1-x}\right]\right), & 0<x \leq 1 \\ 1, & 1<x\end{cases}
$$

With this definition, $H$ vanishes for $E_{2, k}^{* 2} \leq 0$.

Up to this point, we have considered only the on-shell $\mathrm{K}$ matrix, including the analytic continuation to subthreshold momenta. However, in our derivation we also require its off-shell extension, in which the individual particle momenta take on values of $p^{2}$ differing from $m^{2}$. Although not necessary for the implementation of the main result of this work, namely Eq. (1), we find it informative to discuss the off-shell extension of the K matrix. Off-shell extensions are not uniquely defined, as they depend on the choice of single-particle interpolator. In our all-orders diagrammatic analysis, based in a generic effective field theory, we define the fully off-shell scattering amplitude $\mathcal{M}_{2, \text { off,off }}$ by amputating the corresponding four-point correlation function. The presence of two "off"s indicates that both initial and final state particles are off shell. This corresponds to choosing the interpolator to be the fundamental field in the theory, renormalized so that it couples to an on-shell particle with unit amplitude. This is a natural choice in perturbation theory.

In the diagrammatic framework, this definition is naturally extended to the $\mathrm{K}$ matrix. To go from the off-shell
$\mathcal{M}_{2}$ to the off-shell $\mathcal{K}_{2}$, one considers the same amputated correlation function, but replaces the $i \epsilon$ prescription for integrals over poles with the principal value (PV) prescription modified by multiplication by $H(\vec{k})$, as described in Ref. [5]. For our kinematic range, $0<E_{2, k}^{*}<4 m$, this only impacts two-particle intermediate states, and the difference between the prescriptions occurs only when the intermediate state is on shell. This allows one to write the fully off-shell $\mathrm{K}$ matrix in terms of the fully on-shell K matrix,

$$
\begin{aligned}
i \mathcal{K}_{2, \text { off,off }}^{(\ell)}= & i \mathcal{M}_{2 \text {,off,off }}^{(\ell)} \\
& -i \mathcal{M}_{2, \text { off,on }}^{(\ell)} i H \tilde{\rho} \mathcal{K}_{2, \text { on on }}^{(\ell)} \mathcal{M}_{2, \text { on,on }}^{(\ell)-1} i \mathcal{M}_{2, \text { on ,off }}^{(\ell)},
\end{aligned}
$$

where momentum arguments are suppressed for the sake of brevity. We derive this result in Appendix A. As also discussed in the Appendix, it follows from Eq. (13) that the off- and on-shell $\mathrm{K}$ matrices have poles at the same positions, the first of the key results mentioned in the introduction.

We now turn to the case of interest in which $\mathcal{K}_{2 \text {,on,on }}^{(\ell)}$ has a pole for $\ell=J$. Above threshold, this happens when $\cot \delta_{J}$ vanishes, i.e., when the phase shift passes through $\pi / 2+$ $n \pi$ for any integer $n$. If the phase shift is increasing this corresponds to a nearby resonance, but we stress that we must also consider the situation in which $\delta_{J}$ decreases through $\pi / 2+n \pi$, which does not correspond to a resonance but still leads to power-law finite-volume effects. $\mathcal{K}_{2}$ can also have a pole below threshold, when $\mathcal{M}_{2}^{(\ell)-1}=$ $-H \tilde{\rho}$ [see Eq. (9)]. This is not directly associated with anything physical, e.g., a bound state, but nevertheless also contributes finite-volume effects. In all cases, near the pole the on-shell $\mathrm{K}$ matrix has the form

$$
\mathcal{K}_{2, \mathrm{on}, \mathrm{on}}^{(J)}\left(P_{2, k}^{2}\right)=\frac{R}{P_{2, k}^{2}-M^{2}}+\text { nonpole }
$$

with $M$ being the pole position and $R$ a real constant. The pole must have a Lorentz-invariant form as $\mathcal{K}_{2}$ is relativistically invariant. Inserting Eq. (14) into Eq. (13), it follows from the structure of the second term on the righthand side of the latter equation that the off-shell momentum dependence factorizes, as discussed in Appendix A. This allows us to write

$$
\begin{aligned}
& i \mathcal{K}_{2, \mathrm{off}, \mathrm{off}}^{(J)}\left(P_{2, k}^{2} ; p^{2}, b^{2}, a^{\prime 2}, b^{\prime 2}\right) \\
& =\left(p_{2, k}^{*}\right)^{J} i \Gamma_{J}\left(M^{2}, p^{2}, b^{2}\right) \frac{i \eta_{J}}{P_{2, k}^{2}-M^{2}} i \Gamma_{J}\left(M^{2}, a^{2}, b^{\prime 2}\right)\left(a_{2, k}^{\prime *}\right)^{J} \\
& \quad+i \tilde{\mathcal{K}}_{2, \mathrm{off}, \mathrm{off}}^{(J)}\left(P_{2, k}^{2} ; p^{2}, b^{2}, a^{\prime 2}, b^{\prime 2}\right) .
\end{aligned}
$$

Here we have made the momentum arguments explicit: $a^{\prime}$ and $b^{\prime}$ are the incoming 4-momenta, while $p$ and $b$ are the 
outgoing. Factorization manifests itself as the dependence on $a^{\prime 2}$ and $b^{\prime 2}$ being independent of that on $p^{2}$ and $b^{2}$. These dependences arise, respectively, from the factors of $\mathcal{M}_{2, \text { on,off }}$ and $\mathcal{M}_{2, \text { off,on }}$, in Eq. (13). Since they are related by time reversal, the residue functions $\Gamma_{J}$ that carry the offshell dependence are the same for initial and final momenta. These residue functions are real.

The remaining factors in Eq. (15) can be understood as follows: $\eta_{J}= \pm 1$ encodes the sign of the residue, with both values allowed since this is not a physical pole. The $\tilde{\mathcal{K}}_{2}$ term is the nonpole residue and is a smooth function of its arguments. Finally, the "barrier factors" $\left(p_{2, k}^{*}\right)^{J}$ and $\left(a_{2, k}^{\prime *}\right)^{J}$ have been pulled out ${ }^{7}$ so that when $\mathcal{K}_{2 \text {,off,off }}^{(J)}$ is multiplied by spherical harmonics to reconstruct the full $\mathcal{K}_{2 \text {,off,off }}$ there are no nonanalyticities when $p_{2, k}^{*}$ and $a_{2, k}^{\prime *}$ vanish. ${ }^{8}$

We choose in Eq. (15) to set the first argument in $\Gamma_{J}$ (which, in general, is $P_{2, k}^{* 2}$ ) to its value at the pole, $M^{2}$. This choice is convenient for the derivations. It is allowed as the difference cancels the pole and leads to a term that can be absorbed in $\tilde{\mathcal{K}}_{2}$. We stress, however, that we do not evaluate the barrier factors at the pole, since this would reintroduce the nonanalyticities that these factors remove.

Taking the on-shell limit, i.e., sending $p^{2}, b^{2}, a^{\prime 2}$, $b^{\prime 2} \longrightarrow m^{2}$, we reach

$i \mathcal{K}_{2}^{(J)}\left(P_{2, k}^{2}\right)=\left(q_{2, k}^{*}\right)^{J} i \Gamma_{J} \frac{i \eta_{J}}{P_{2, k}^{2}-M^{2}} i \Gamma_{J}\left(q_{2, k}^{*}\right)^{J}+i \tilde{\mathcal{K}}_{2}^{(J)}\left(P_{2, k}^{2}\right)$,

where we have introduced the following shorthand for the fully on-shell residue function,

$$
\Gamma_{J} \equiv \Gamma_{J}\left(M^{2}, m^{2}, m^{2}\right),
$$

together with analogous notation for $\mathcal{K}_{2}^{(J)}$ and $\tilde{\mathcal{K}}_{2}^{(J)}$. As is shown explicitly in the following subsection, the quantities $\mathcal{K}_{2}^{(\ell)}, \Gamma_{J}$ and $M^{2}$ all enter the definition of the finite-volume matrix $\mathcal{F}$.

\section{Definition of $\mathcal{F}$}

We now have the ingredients necessary to define the entries in the matrix $\mathcal{F}$, Eq. (3). We begin with the 33 component, $F_{33}=F_{33 ; k^{\prime} \ell^{\prime} m^{\prime} ; k \ell m}$. This is defined by

\footnotetext{
${ }^{7}$ The quantities $a_{2, k}^{\prime *}$ and $p_{2, k}^{*}$ are similar to the $a_{2, k}^{*}$ defined in Table I. They are obtained by boosting the 4-momenta $a^{\prime}$ and $p$, respectively, into the two-particle c.m. frame. They differ from $a_{2, k}^{*}$ slightly because $a^{\prime}$ and $p$ are not, in general, on shell 4-vectors, while, in Table I, $a$ is on shell.

${ }^{8}$ The key point here is that $a^{\ell} Y_{\ell m}(\hat{a})$ is a polynomial in the components of $\vec{a}$, while $Y_{\ell m}(\hat{a})$ is nonanalytic at $\vec{a}=0$.
}

$$
i F_{33} \equiv \frac{1}{2 \omega L^{3}}\left[\frac{i F}{3}+i F i \mathcal{T}_{L} i F\right]
$$

where

$$
i \mathcal{T}_{L} \equiv \frac{1}{1-i \mathcal{K}_{2}(i F+i G)} i \mathcal{K}_{2}
$$

and

$$
\begin{gathered}
{\left[\frac{1}{2 \omega L^{3}}\right]_{k^{\prime}, \ell^{\prime}, m^{\prime} ; k, \ell, m} \equiv \delta_{k^{\prime}, k} \delta_{\ell^{\prime}, \ell} \delta_{m^{\prime}, m} \frac{1}{2 \omega_{k} L^{3}},} \\
i G_{p, \ell^{\prime}, m^{\prime} ; k, \ell, m} \equiv \mathcal{Y}_{3, \ell^{\prime} m^{\prime}}\left(\vec{k}_{2, p}^{*}\right) i \mathbf{S}_{3}^{0}(\vec{p}, \vec{k}) \mathcal{Y}_{3, \ell m}^{*}\left(\vec{p}_{2, k}^{*}\right) \frac{1}{2 \omega_{k} L^{3}}, \\
i F_{k^{\prime}, \ell^{\prime}, m^{\prime} ; k, \ell, m} \equiv \delta_{k^{\prime}, k} i F_{\ell^{\prime}, m^{\prime} ; \ell, m}(\vec{k}), \\
i F_{\ell^{\prime}, m^{\prime} ; \ell, m}(\vec{k}) \equiv i F_{\ell^{\prime}, m^{\prime} ; \ell, m}^{i \epsilon}(\vec{k})+i \rho_{\ell^{\prime}, m^{\prime} ; \ell, m}(\vec{k}), \\
i F_{\ell^{\prime}, m^{\prime} ; \ell, m}^{i \epsilon}(\vec{k}) \equiv \frac{1}{2}\left[\frac{1}{L^{3}} \sum_{\vec{a}}-\int_{\vec{a}}\right] \\
\times \frac{1}{2 \omega_{a}} \mathcal{Y}_{3, \ell^{\prime} m^{\prime}}\left(\vec{a}_{2, k}^{*}\right) i \mathbf{S}_{3}^{i \epsilon}(\vec{p}, \vec{k}) \mathcal{Y}_{3, \ell m}^{*}\left(\vec{a}_{2, k}^{*}\right),
\end{gathered}
$$

$$
i \mathcal{K}_{2 ; k^{\prime} \ell^{\prime} m^{\prime} ; k \ell m} \equiv \delta_{k^{\prime}, k} \delta_{\ell^{\prime}, \ell} \delta_{m^{\prime}, m} i \mathcal{K}_{2}^{(\ell)}\left(P_{2, k}^{2}\right),
$$

with $\int_{\vec{a}} \equiv \int d^{3} a /(2 \pi)^{3}$ and $\sum_{\vec{a}}=\sum_{\vec{n} \in \mathbb{Z}^{3}, \vec{a}=2 \pi \vec{n} / L}$. We have introduced a compact notation for poles and harmonic polynomials

$$
\begin{aligned}
\mathcal{Y}_{3, \ell m}\left(\vec{k}_{2, p}^{*}\right) & \equiv \sqrt{4 \pi}\left(\frac{k_{2, p}^{*}}{q_{2, p}^{*}}\right)^{\ell} Y_{\ell m}\left(\hat{k}_{2, p}^{*}\right), \\
i \mathbf{S}_{3}^{i \epsilon}(\vec{p}, \vec{k}) & \equiv \frac{i H_{3}(\vec{p}, \vec{k})}{b_{p k}^{2}-m^{2}+i \epsilon} .
\end{aligned}
$$

In Eq. (23), $\rho(\vec{k})$ is a phase-space factor defined by

$$
\rho_{\ell^{\prime}, m^{\prime} ; \ell, m}(\vec{k}) \equiv \delta_{\ell^{\prime}, \ell} \delta_{m^{\prime}, m} H(\vec{k}) \tilde{\rho}\left(P_{2, k}\right)
$$

where $\tilde{\rho}\left(P_{2, k}^{2}\right)$ and $H(\vec{k})$ are defined in Eqs. (10) and (11) above. Finally, $H_{3}(\vec{p}, \vec{k})$ is a symmetric product of the smooth cutoff function, $H(\vec{k})$,

$$
H_{3}(\vec{p}, \vec{k})=H(\vec{k}) H(\vec{p}) H\left(\vec{b}_{p k}\right)
$$

This definition is nearly the same as that used in Refs. [5,6]. There are two differences. The first is that 
$F$ and $G$ are expressed here in a manifestly Lorentz covariant way-the pole term in $\mathbf{S}_{3}$ involves the square of a 4 -vector rather than the energies in the finite-volume frame. This changes $F$ only by exponentially suppressed contributions, but for $G$ the modification is significant. In particular, using the definition above leads to $\mathcal{K}_{\mathrm{df}}$ being a Lorentz scalar, as noted in Ref. [7]. The second change is that, in Refs. [5,6], $G$ is defined with $H_{3} \rightarrow H(\vec{k}) H(\vec{p})$ rather than the form with three $H$ functions given in Eq. (28). The present definition is that which appears in the case of no $\mathbb{Z}_{2}$ symmetry, as shown in Ref. [16]. Thus, although it is not mandatory here, this choice of $\mathrm{H}_{3}$ seems more likely to lead to a formalism that smoothly goes over to the result when the resonance becomes stable. In any case, it is one possible choice.

The other three entries of $\mathcal{F}$ are new to this work, and are all brought about by the presence of the pole in $\mathcal{K}_{2}$. They are defined as

$$
\begin{aligned}
i F_{\tilde{2} \tilde{2}} \equiv & i F_{\rho \pi}+i G_{\rho} i \Gamma_{J} \frac{1}{2 \omega L^{3}}(i F+i G) \\
& \times \frac{1}{1-i \mathcal{K}_{2}(i F+i G)} i \Gamma_{J} i G_{\rho}^{\dagger}, \\
i F_{\tilde{2} 3} \equiv & i G_{\rho} i \Gamma_{J} \frac{1}{2 \omega L^{3}} \frac{1}{1-(i F+i G) i \mathcal{K}_{2}} i F \\
i F_{3 \tilde{2}} \equiv & \frac{1}{2 \omega L^{3}} i F \frac{1}{1-i \mathcal{K}_{2}(i F+i G)} i \Gamma_{J} i G_{\rho}^{\dagger},
\end{aligned}
$$

where $\Gamma_{J}$ is the on-shell residue defined in Eq. (17), and we have introduced two new kinematic functions, needed to describe the finite-volume dependence arising from the K-matrix pole,

$$
\begin{gathered}
i G_{\rho ; M_{J}^{\prime} \ell^{\prime} m^{\prime} ; k \ell m} \equiv \mathcal{Y}_{\tilde{2}, \ell^{\prime} m^{\prime}}\left(\vec{k}^{*}\right) i \mathbf{S}_{\tilde{2}}(\vec{k}) \delta_{J, \ell} \delta_{M_{J}^{\prime}, m}\left(q_{2, k}^{*}\right)^{J}, \\
i F_{\rho \pi ; M_{J}^{\prime} \ell^{\prime} m^{\prime} ; M_{J} \ell m} \\
\equiv \delta_{M_{J}^{\prime} M_{J}} \frac{1}{L^{3}} \sum_{\vec{k}} \frac{1}{2 \omega_{k}} \mathcal{Y}_{\tilde{2}, \ell^{\prime} m^{\prime}}\left(\vec{k}^{*}\right) i \mathbf{S}_{\tilde{2}}(\vec{k}) \mathcal{Y}_{\tilde{2}, \ell m}^{*}\left(\vec{k}^{*}\right), \\
\mathcal{Y}_{\tilde{2}, \ell m}\left(\vec{k}^{*}\right) \equiv \sqrt{4 \pi}\left(\frac{k^{*}}{q_{\rho}^{*}}\right)^{\ell} Y_{\ell m}\left(\hat{k}^{*}\right), \\
i \mathbf{S}_{\tilde{2}}(\vec{k}) \equiv \frac{i \eta_{J} H_{\rho}(\vec{k})}{P_{2, k}^{2}-M^{2}}
\end{gathered}
$$

where $\eta_{J}= \pm 1$ encodes the sign of the residue of the $\mathcal{K}_{2}$ pole and is defined in Eq. (15).

Here we require an additional cutoff function, $H_{\rho}(\vec{k})$, the role of which is to provide an ultraviolet cutoff for the sum in Eq. (33). ${ }^{9}$ The range of possible $\mathcal{K}_{2}$ pole masses that we need to accommodate is $0<M<4 m$, with the lower limit set by the value of $P_{2, k}^{2}$ for which $H(\vec{k})$ vanishes, and the upper limit set by the opening of the five-particle threshold with respect to $E^{*}$. For any choice of $\vec{k}$ such that $P_{2, k}^{2}$ lies in this range, we need $H_{\rho}(\vec{k})=1$, so as not to distort the pole. However, as $P_{2, k}^{2}$ drops below 0 , the cutoff function should smoothly drop to 0 . The detailed choice is not important, but we display one example for illustration,

$$
H_{\rho}(\vec{k})=J\left(\frac{P_{2, k}^{* 2}+4 m^{2}}{4 m^{2}}\right) \text {. }
$$

This is chosen so that $H_{\rho}$ vanishes when $P_{2, k}^{* 2} \leq-4 m^{2}$.

One of the important properties of $F_{33}$, stressed in Refs. [5,6], is that it is fully determined if one knows $\mathcal{K}_{2}$ in the relevant kinematic range. Thus a separate study using the two-particle quantization condition can, in principle, determine the finite-volume function. We stress here that the same is true for all four components of $\mathcal{F}$. The only difference is that we must pull out the pole contribution from $\mathcal{K}_{2}$, and use this in the determination of $F_{32}$, $F_{\tilde{2} 3}$ and $F_{\tilde{2} \tilde{2}}$. The added complexity when there is a pole in $\mathcal{K}_{2}^{(J)}$ manifests only in the way that information about twoparticle interactions appears in the finite-volume functions.

\section{Definition of $\mathcal{K}_{\mathrm{df}}$}

We close this section with some brief comments on $\mathcal{K}_{\text {df }}$, whose components are given by Eq. (2). These four entries $\left(\mathcal{K}_{\mathrm{df}, \tilde{2} \tilde{2}}, \mathcal{K}_{\mathrm{df}, \tilde{2} 3}, \mathcal{K}_{\mathrm{df}, \tilde{3} \tilde{2}}\right.$ and $\left.\mathcal{K}_{\mathrm{df}, 33}\right)$ are each infinite-volume quantities, characterizing scattering in the indicated channels. They are, themselves, matrices with indices matching those of the corresponding components of $\mathcal{F}$, Eq. (3). When multiplied by the appropriate spherical harmonics, and summed over angular momentum indices, they become real, Lorentz-invariant functions of the on-shell kinematic variables.

Another key property is that, in each of the four components, all kinematic singularities and possible $\mathcal{K}_{2}$ poles have been removed from $\mathcal{K}_{\mathrm{df}}$. Thus, these can be viewed in position space as quasilocal vertices connecting the various channels. This analogy is not perfect, however, since the components of $\mathcal{K}_{\mathrm{df}}$ are not physical, as they depend on the details of the cutoff functions described above. It is also possible, just as for $\mathcal{K}_{2}$, that there are dynamical singularities in $\mathcal{K}_{\mathrm{df}}$.

\footnotetext{
${ }^{9} H_{\rho}(\vec{k})$ also appears in $G_{\rho}$ through the pole factor, $\mathbf{S}_{\tilde{2}}$. In fact, here the cutoff function has no effect because $G_{\rho}$ is always accompanied by $F$ or $G$ and thus $H_{\rho}(\vec{k})$ is always multiplied by $H(\vec{k})$. From the definitions of the cutoff function it trivially follows that $H_{\rho}(\vec{k}) H(\vec{k})=H(\vec{k})$. We nonetheless find it convenient to keep the cutoff within $G_{\rho}$ as written.
} 
The derivation presented in the next section provides a complicated and implicit definition of the components of $\mathcal{K}_{\mathrm{df}}$. This turns out to be sufficient, however, because what really matters is how these components are related to the physically measurable three-to-three scattering amplitude. This relation can be derived based solely on how $\mathcal{K}_{\mathrm{df}}$ enters the final result. This is presented in Sec. IV, following the approach of Ref. [6].

\section{DERIVATION}

We now derive the result described in the previous section. Begin by defining a finite-volume correlation function

$$
C_{L}(E, \vec{P}) \equiv-\int_{L} d^{4} x e^{-i E t+i \vec{P} \cdot \vec{x}}\left\langle\Omega\left|\mathrm{T} \mathcal{O}(x) \mathcal{O}^{\dagger}(0)\right| \Omega\right\rangle,
$$

where $\mathcal{O}^{\dagger}(0)$ is any operator with the quantum numbers of the three-particle states that we are after. ${ }^{10}$ Inserting a complete set of states, one can show that this object has poles in $E$ at the finite-volume energies. Our aim is thus to derive an equation - the quantization condition-for the locations of these poles.

In the following subsections we show that the correlator can be written as

$$
C_{L}(E, \vec{P})=C_{\infty}(E, \vec{P})+i A^{\prime} i \mathcal{F} \frac{1}{1-i \mathcal{K}_{\mathrm{df}} i \mathcal{F}} i A,
$$

up to exponentially suppressed corrections. Here $C_{\infty}(E, \vec{P})$ and

$$
i A^{\prime} \equiv\left(\begin{array}{ll}
i A_{\tilde{2}}^{\prime} & i A_{3}^{\prime}
\end{array}\right), \quad i A \equiv\left(\begin{array}{c}
i A_{\tilde{2}} \\
i A_{3}
\end{array}\right)
$$

are infinite-volume quantities, defined in the course of the following subsections. Note that the second term in Eq. (38) is a product of a row vector, a matrix, and a column vector, with all indices contracted. As the infinitevolume quantities contain no finite-volume poles, the poles in $C_{L}(E, \vec{P})$ correspond to divergent eigenvalues in the matrix between $A^{\prime}$ and $A$. This is equivalent to the inverse of the matrix having a vanishing determinant, and thus to the quantization condition given in Eq. (1) above.

\section{A. Compact notation for the derivation}

In order to make the following derivation more readable, in this section we introduce a compact notation for the various quantities introduce above. Our aim is to minimize explicit factors of $i$ and of $2 \omega L^{3}$. We thus define

\footnotetext{
${ }^{10}$ The overall minus sign included in this definition should be understood as a factor of $i^{2}$. We choose to accompany each operator with a factor of $i$ for reasons explained below.
}

$$
\begin{gathered}
\mathbf{G} \equiv \frac{1}{2 \omega L^{3}} i G, \quad \mathbf{F} \equiv \frac{1}{2 \omega L^{3}} i F, \quad \mathbf{K}_{2} \equiv 2 \omega_{k} L^{3} i \mathcal{K}_{2}, \\
\boldsymbol{\Gamma} \equiv i \Gamma_{J}, \quad \mathbf{G}_{\rho} \equiv i G_{\rho}, \quad \overline{\mathbf{G}}_{\rho} \equiv i G_{\rho}^{\dagger}, \quad \mathbf{F}_{\rho \pi} \equiv i F_{\rho \pi} .
\end{gathered}
$$

One advantage of these definitions is that $\mathbf{G}$ is now given by

$$
\begin{aligned}
\mathbf{G}_{p, \ell^{\prime}, m^{\prime} ; k, \ell, m} \equiv & \frac{1}{2 \omega L^{3}} i G_{p, \ell^{\prime}, m^{\prime} ; k, \ell, m} \\
\equiv & \frac{1}{2 \omega_{p} L^{3}} \mathcal{Y}_{3, \ell^{\prime} m^{\prime}}\left(\vec{k}_{2, p}^{*}\right) i \mathbf{S}_{3}^{0}(\vec{p}, \vec{k}) \mathcal{Y}_{3, \ell m}^{*}\left(\vec{p}_{2, k}^{*}\right) \\
& \times \frac{1}{2 \omega_{k} L^{3}},
\end{aligned}
$$

and is therefore anti-Hermitian (due to the factor of $i$ in the definition). This avoids the need to define the separate object $\left[2 \omega L^{3}\right]^{-1} G\left[2 \omega L^{3}\right]$ that would otherwise appear in the derivation.

In this new notation, the quantization condition becomes

$$
\operatorname{det}\left[1-\mathbf{K}_{\mathrm{df}}\left(E^{*}\right) \mathcal{F}(E, \vec{P}, L)\right]=0,
$$

where

$$
\begin{aligned}
i \mathcal{K}_{\mathrm{df}} & \equiv \mathbf{K}_{\mathrm{df}} \equiv\left(\begin{array}{ll}
\mathbf{K}_{\mathrm{df}, \tilde{2} \tilde{2}} & \mathbf{K}_{\mathrm{df}, \tilde{2} 3} \\
\mathbf{K}_{\mathrm{df}, 3 \tilde{2}} & \mathbf{K}_{\mathrm{df}, 33}
\end{array}\right), \\
i \mathcal{F} & \equiv \mathcal{F} \equiv\left(\begin{array}{ll}
\mathbf{F}_{\tilde{2} \tilde{2}} & \mathbf{F}_{\tilde{2} 3} \\
\mathbf{F}_{3 \tilde{2}} & \mathbf{F}_{33}
\end{array}\right),
\end{aligned}
$$

and

$$
\begin{gathered}
\mathbf{F}_{33}=\frac{1}{3} \mathbf{F}+\mathbf{F T}_{L} \mathbf{F}, \quad \text { with } \quad \mathbf{T}_{L} \equiv \frac{1}{1-\mathbf{K}_{2}(\mathbf{F}+\mathbf{G})} \mathbf{K}_{2}, \\
\mathbf{F}_{\tilde{2} \tilde{2}}=\mathbf{F}_{\rho \pi}+\mathbf{G}_{\rho} \boldsymbol{\Gamma}(\mathbf{F}+\mathbf{G}) \frac{1}{1-\mathbf{K}_{2}(\mathbf{F}+\mathbf{G})} \boldsymbol{\Gamma} \overline{\mathbf{G}}_{\rho} \\
\mathbf{F}_{\tilde{2} 3}=\mathbf{G}_{\rho} \boldsymbol{\Gamma} \frac{1}{1-(\mathbf{F}+\mathbf{G}) \mathbf{K}_{2}} \mathbf{F} \\
\mathbf{F}_{3 \tilde{2}}=\mathbf{F} \frac{1}{1-\mathbf{K}_{2}(\mathbf{F}+\mathbf{G})} \boldsymbol{\Gamma} \overline{\mathbf{G}}_{\rho}
\end{gathered}
$$

\section{B. Definition and decomposition of $C_{L}^{\left[B_{2}\right]}$}

We begin by following the same steps as taken by Ref. [5] in the derivation of the quantization condition in the absence of $\mathcal{K}_{2}$ poles. This allows us to reuse a fair amount of work from that reference. The derivation begins with an all-orders skeleton expansion in which $C_{L}$ is defined diagrammatically in terms of two- and three-particle Bethe-Salpeter kernels, denoted $i B_{2}$ and $i B_{3}$ respectively, as well as fully dressed propagators. Examples are shown in Fig. 4 of Ref. [5]. The skeleton expansion is designed to make all power-law finite-volume effects explicit. Such effects arise from on-shell intermediate states in Feynman diagrams and, since we constrain the 
overall c.m. energy to the range $m<E^{*}<5 m$, this amounts to keeping track of three-particle states. The restriction to a finite, periodic spatial volume is effected by summing the spatial components of all loop momenta over $\vec{p}=(2 \pi / L) \vec{n}$ where $\vec{n} \in \mathbb{Z}^{3}$ runs over all 3-vectors of integers.

As in Ref. [5], the challenging part of the derivation is that involving the kernels $B_{2}$. Thus it is useful to begin by analyzing a reduced correlator, denoted $C_{L}^{\left[B_{2}\right]}$, defined by the same skeleton expansion except that all three-particle kernels are set to $0\left(B_{3} \rightarrow 0\right)$. Adding back in the effects of $B_{3}$ is relatively straightforward and will be done at a later stage. To decompose $C_{L}^{\left[B_{2}\right]}$ we can piggyback on Ref. [5] by directly taking over Eq. (174) of that work, since this equation was derived without assuming smoothness of $\mathcal{K}_{2}$ as a function of the two-particle center-of-mass energy. Written in our present notation, the result is

$$
\begin{aligned}
C_{L}^{\left[B_{2}\right]}= & C_{L, 0 F}-\frac{2}{3} \boldsymbol{\sigma}^{*} \mathbf{F} \boldsymbol{\sigma}^{\dagger *} \\
& +\mathbf{A}_{L, 3}^{\prime(u)} \mathbf{F}_{33}^{(0)} \sum_{n=0}^{\infty}\left(\mathbf{K}_{L, 33}^{(u, u)} \mathbf{F}_{33}^{(0)}\right)^{n} \mathbf{A}_{L, 3}^{(u)},
\end{aligned}
$$

where the quantity called $[\mathcal{A}]$ in Ref. [5] is here denoted $\mathbf{F}_{33}^{(0)}$, and is given by

$$
\mathbf{F}_{33}^{(0)} \equiv \mathbf{F} \frac{1}{1-\mathbf{K}_{2} \mathbf{F}} .
$$

The other quantities in Eq. (49) are explained shortly.

What has been achieved in Eq. (49) is to make explicit a subset of the finite-volume effects due to three-particle intermediate states. We recall from Eqs. (24) and (41) that $\mathbf{F}$ is defined by a sum-integral difference of a quantity with a three-particle pole. This object therefore has power-law finite-volume dependence, and also sets the quantities multiplying it on either side to be on shell. Thus $\mathbf{F}_{33}^{(0)}$ collects such dependence from a sequence of three-particle "cuts" separated by two-to-two interactions occurring between the same pair. The subscript " 33 " is included here to distinguish this object from similar quantities involving $\mathcal{K}_{2}$ poles that arise below.

We now turn to the definitions of the remaining quantities in Eq. (49). With the exception of $\boldsymbol{\sigma}^{*}$ and $\boldsymbol{\sigma}^{\dagger *}$, these are finite-volume quantities, involving some loops in which momenta are summed rather than integrated. ${ }^{11}$ This is indicated by the subscripts $L$.

We begin with $C_{L, 0 F}$. This is the contribution to $C_{L}^{\left[B_{2}\right]}$ containing no factors of $\mathbf{F}$. It can be expanded according to the number of factors of $\mathcal{K}_{2}$ that it contains,

$$
C_{L, 0 F}=\sum_{n=0}^{\infty} C_{L, 0 F}^{(n)}
$$

\footnotetext{
${ }^{11}$ Here and in the following a loop momentum being summed is shorthand for a sum over spatial components and an integral over the temporal component.
}

The objects on the right-hand side are identical to those with the same names appearing in Ref. [5]. They are defined in Eqs. (114), (154), (169), (173) and (176) of that work, and shown diagrammatically there in Figs. 11(c), 15(b) and 17(c). We repeat the diagrammatic representation in Fig. 1(a) below.

The quantity $\mathbf{K}_{L, 33}^{(u, u)}$ involves three-to-three transitions that are built from $\mathcal{K}_{2}$ interactions alternating between different pairs. It is closely related to $\mathcal{K}_{3, L}^{(n, u, u)}$, defined in Ref. [5],

$$
\mathbf{K}_{L, 33}^{(u, u)} \equiv \sum_{n=2}^{\infty} \mathbf{K}_{L, 33}^{(n, u, u)}, \quad \mathbf{K}_{L, 33}^{(n, u, u)} \equiv i \mathcal{K}_{3, L}^{(n, u, u)}
$$

Here $\mathbf{K}_{L, 33}^{(n, u)}$ is the contribution containing $n$ factors of $\mathcal{K}_{2}$, where $n \geq 2$. As above, we have amended the subscripts to facilitate the addition of the $\rho \pi$ channel, and also absorbed a factor of $i$. The definition of $\mathcal{K}_{3, L}^{(n, u, u)}$ is given in Ref. [5] by Eqs. (155) and (171) and Figs. 11(a), 15(c) and 17(c). We repeat the diagrammatic representation of $\mathbf{K}_{L, 33}^{(n, u, u)}$ in Fig. 2(a) below.

The remaining quantities in Eq. (49) are end caps. $\mathbf{A}_{L, 3}^{\prime(u)}$ and $\mathbf{A}_{L, 3}^{(u)}$ can be expanded as above according to the number of factors of $\mathcal{K}_{2}$, with $\boldsymbol{\sigma}^{*}$ and $\boldsymbol{\sigma}^{\dagger *}$ being the zeroth order terms in these expansions,

$$
\begin{aligned}
\mathbf{A}_{L, 3}^{\prime(u)} & =\sum_{n=0}^{\infty} \mathbf{A}_{L, 3}^{\prime(n, u)}, \quad \mathbf{A}_{L, 3}^{(u)}=\sum_{n=0}^{\infty} \mathbf{A}_{L, 3}^{(n, u)}, \\
\boldsymbol{\sigma}^{*} & \equiv \mathbf{A}_{L, 3}^{\prime(0, u)}, \quad \boldsymbol{\sigma}^{\dagger *} \equiv \mathbf{A}_{L, 3}^{(0, u)} .
\end{aligned}
$$

The relation to the corresponding quantities from Ref. [5] simply involves a change of subscripts to allow for future $\mathcal{K}_{2}$ pole contributions,

$$
\mathbf{A}_{L, 3}^{\prime(n, u)} \equiv i A_{L}^{\prime(n, u)}, \quad \mathbf{A}_{L, 3}^{(n, u)} \equiv i A_{L}^{(n, u)},
$$

where the quantities on the right-hand side are those appearing in Ref. [5].

The expressions for these quantities are given in Eqs. (60), (84), (85), (113), (153) and (170) of Ref. [5], and illustrated in Figs. 9(c), 11(b), and 17(a) of that work. We repeat the diagrammatic representation of the left end cap $\mathbf{A}_{L, 3}^{\prime(n, u)}$ in Fig. 1(c) below. Note that it can be obtained from that for $C_{L, 0 F}^{(n)}$ by removing the $\boldsymbol{\sigma}^{\dagger *}$ at the right end. The representation of the corresponding right end cap, $\mathbf{A}_{L, 3}^{(u)}$, is given simply by a horizontal reflection of that for $\mathbf{A}_{L, 3}^{\prime(u)}$, or equivalently by removing $\boldsymbol{\sigma}^{*}$ from the left end of $C_{L, 0 F}^{(n)}$.

In the following subsections, our aim is to make explicit the full volume dependence of $C_{L, 0 F}, \mathbf{A}_{L, 3}^{\prime(u)}, \mathbf{A}_{L, 3}^{(u)}$ and $\mathbf{K}_{L, 33}^{(u, u)}$. 
(a)
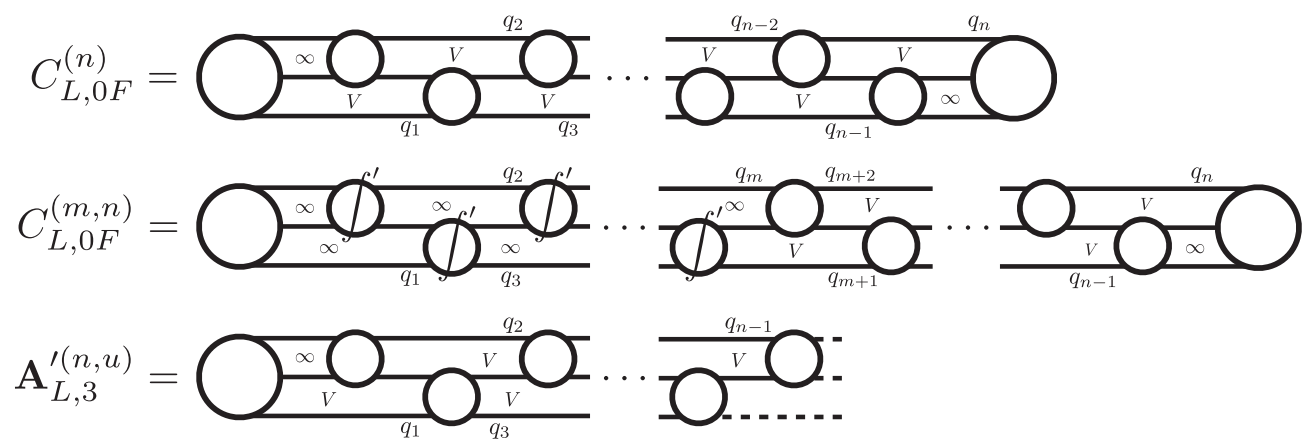

(d)
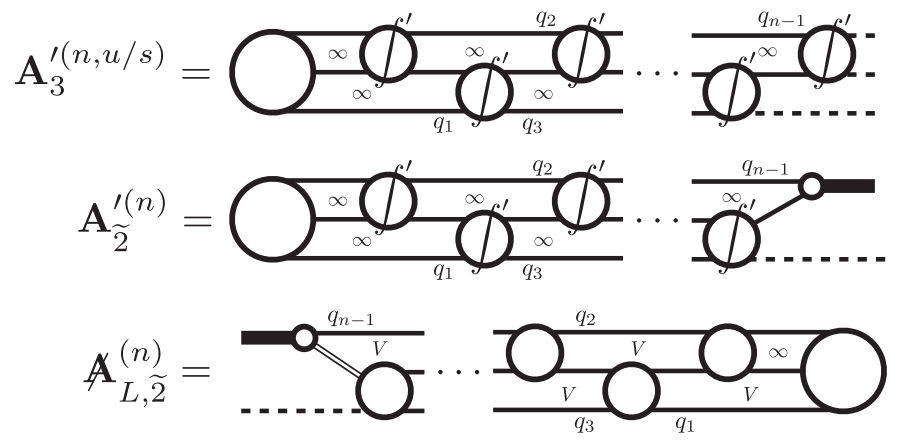

FIG. 1. Diagrammatic definitions of quantities entering the derivation of Eq. (55). Open circles on the left and right ends represent $\sigma^{*}$ and $\boldsymbol{\sigma}^{\dagger *}$, respectively. Open circles in the middle represent the full off-shell $i \mathcal{K}_{2}$, while the circles with an integral sign indicate that only the smooth component, $i \tilde{\mathcal{K}}_{2}^{\prime}$, is included. Loops that are summed contain a " $V$," while those that are integrated contain an " $\infty$." The superscript $n$ indicates the number of factors of $\mathcal{K}_{2}$ or its smooth counterpart. Thin lines are fully dressed propagators, with unit residue at the one-particle pole. Thick lines [present in (e) and (f)] represent the resonance, corresponding to the subscript $\tilde{2}$. Double thin lines [present only in (f)] indicate that only the smooth part of the exchanged particle propagator is kept. In (c), the superscript " $u$ " indicates that the index $k$ corresponds to the momentum carried by the spectator propagator at the right-hand end. In (d), the superscript " $u / s$ " indicates that the diagram serves to define the quantity with both superscripts. If the superscript is $u$, then the momentum $k$ is assigned to the spectator propagator, while if it is $s$, then $k$ is assigned to the upper propagator. Further details are given in the text.

\section{Decomposition of $C_{L, 0 F}$}

We first consider the quantity $C_{L, 0 F}$, and show in this subsection that it can be decomposed as

$$
\begin{aligned}
C_{L, 0 F}= & C_{\infty}^{\left[B_{2}\right]}+\left(2 \mathbf{A}_{3}^{\prime(s)} \mathbf{F}+\mathbf{A}_{\tilde{2}}^{\prime} \mathbf{G}_{\rho} \mathbf{\Gamma} \mathbf{G}\right)\left(\mathbf{A}_{L, 3}^{(u)}-\boldsymbol{\sigma}^{\dagger *}\right) \\
& +\mathbf{A}_{\tilde{2}}^{\prime} \mathbf{F}_{\rho \pi} \mathbf{A}_{L, \tilde{2}} .
\end{aligned}
$$

This is a partial decomposition, involving both finiteand infinite-volume quantities (the latter having subscripts including $L$ ) separated by cuts. In deriving this result, we must, for the first time, account for the poles in $\mathcal{K}_{2}$, as shown by the presence of factors of $\mathbf{G}_{\rho}$ and $\mathbf{F}_{\rho \pi}$, which set the $\rho \pi$ states on either side on shell. If these two quantities are set to 0 we reproduce the result in Eq. (189) of Ref. [5].

As can be seen from Fig. 1(a), $C_{L, 0 F}$ is defined by the sum over all pairwise scatterings in which the interaction switches to a different pair with each new insertion of $i \mathcal{K}_{2}$. By construction, this quantity has $n$ summed loop momenta plus two additional loops with integrated momenta. It is convenient to extend this notation by defining $C_{L, 0 F}^{(m, n)}$ to be the same quantity as $C_{L, 0 F}^{(n)}$ but with the leftmost $m$ momentum sums converted to integrals (with the singleparticle poles integrated using the $\widetilde{\mathrm{PV}}$ prescription of Ref. [5]), and with the integrated $i \mathcal{K}_{2}$ factors replaced by their smooth parts, $i \tilde{\mathcal{K}}_{2}^{\prime}$ [see Fig. 1(b)]. ${ }^{12}$ This requires $0 \leq$ $m \leq n$, with $m=0$ leading to $C_{L, 0 F}^{(0, n)} \equiv C_{L, 0 F}^{(n)}$, and $m=n$ to $C_{L, 0 F}^{(n, n)} \equiv C_{\infty}^{(n)}$, a fully integrated, infinite-volume quantity.

To decompose $C_{L, 0 F}^{(m, n)}$ we consider the leftmost sum, i.e., that directly adjacent to the $m$ integrated loops. Finitevolume effects arise in this sum due to both the pole in $\mathcal{K}_{2}$ and the intermediate on-shell three-particle state. In Appendix B 1 we explain the procedure for converting a given summed loop (with the full $\mathcal{K}_{2}$ ) to an integrated loop (with the smooth part only). In other words we derive a system for converting $C_{L, 0 F}^{(m, n)}$ to $C_{L, 0 F}^{(m+1, n)}$, plus finite-volume correction terms. This leads to the following recursion relation:

\footnotetext{
${ }^{12}$ As explained in Appendix B 1, several terms combine to give the smooth part, with $i \tilde{\mathcal{K}}_{2}$ from Eq. (B4) being just one contribution. This is illustrated in Fig. 7(f). It is for this reason that we require the prime to denote, $i \tilde{\mathcal{K}}_{2}^{\prime}$, the quantity entering integrated loops of $C_{L, 0 F}^{(m, n)}$.
} 


$$
C_{L, 0 F}^{(m, n)}= \begin{cases}C_{L, 0 F}^{(m+1, n)}+\left(2 \mathbf{A}_{3}^{\prime(m+1, s)} \mathbf{F}+\mathbf{A}_{2}^{\prime(m+1)} \mathbf{G}_{\rho} \mathbf{\Gamma G}\right) \mathbf{A}_{L, 3}^{(n-m-1, u)}+\mathbf{A}_{2}^{\prime(m+1)} \mathbf{F}_{\rho \pi} \mathbf{A}_{L, \tilde{2}}^{(n-m)} & 0 \leq m<n-1, \\ C_{L, 0 F}^{(n, n)}+\mathbf{A}_{2}^{\prime(n)} \mathbf{F}_{\rho \pi} \mathbf{A}_{L, \tilde{2}}^{(1)} & 0 \leq m=n-1, \\ C_{\infty}^{(n)} & 0 \leq m=n .\end{cases}
$$

The pole in $\mathcal{K}_{2}$ leads to the terms involving $\mathbf{G}_{\rho}$ and $\mathbf{F}_{\rho \pi}$. These equations contain three new quantities, $\mathbf{A}_{3}^{\prime(n, s)}, \mathbf{A}_{\tilde{2}}^{(n)}$ and $\mathbf{A}_{L, \tilde{2}}^{(n)}$, in addition to the right end cap $\mathbf{A}_{L, 3}^{(n, u)}$ introduced in the previous subsection.

The infinite-volume left end cap $\mathbf{A}_{3}^{\prime(n, s)}$ is defined diagrammatically in Fig. 1(d). It contains $n$ factors of $i \tilde{\mathcal{K}}_{2}^{\prime}$, with all loop momenta integrated. The superscript $s$ indicates the manner in which the on-shell external threeparticle state is projected into spherical harmonics, as explained in Ref. [5].

The second new quantity is the infinite-volume left end cap $\mathbf{A}_{\tilde{2}}^{\prime(n+1)}$, defined diagrammatically in Fig. 1(e). Here the on-shell external state consists of the K-matrix pole plus the spectator, which we refer to as the $\rho \pi$ state. $\mathbf{A}_{\tilde{2}}^{\prime(n+1)}$ contains $n$ factors of $\tilde{\mathcal{K}}_{2}^{\prime}, n$ loop integrals, and one factor of $\boldsymbol{\Gamma}$ in the loop adjacent to the external state. We later need the analogous right end cap, denoted $\mathbf{A}_{\tilde{2}}^{(n+1)}$.

The final new quantity, $\mathbf{A}_{L, \tilde{2}}^{(n+1)}$, is defined diagrammatically in Fig. 1(f). It is closely related to $\mathbf{A}_{L, 2}^{(n+1)}$, the reflection of $\mathbf{A}_{L, 2}^{\prime(n+1)}$, which consists of $n$ factors of $i \mathcal{K}_{2}$, $n$ summed loops, and one factor of $\boldsymbol{\Gamma}$ adjacent to the external $\rho \pi$ state. The slashed version differs in that the leftmost three-particle intermediate state is replaced by the smooth difference that remains when the $\mathbf{G}$ singularity is subtracted. This subtraction in indicated in the figure with a double line. The lowest value of $n, n=1$, is a special case, for which there is no summed loop and $\mathbf{A}_{L, \tilde{2}}^{(1)}=\mathbf{A}_{\tilde{2}}^{(1)}$. For further discussion, see Appendix B 1.

Iterating Eq. (56) leads to an expression for $C_{L, 0 F}^{(0, n)}=$ $C_{L, 0 F}^{(n)}$ in terms of $C_{\infty}^{(n)}$. Summing over $n$ then gives the desired result, Eq. (55), where we define

$$
\begin{aligned}
C_{\infty}^{\left[B_{2}\right]} & =\sum_{n=0}^{\infty} C_{\infty}^{(n)}, & \mathbf{A}_{3}^{\prime(s)} & =\sum_{n=1}^{\infty} \mathbf{A}_{3}^{\prime(n, s)}, \\
\mathbf{A}_{\tilde{2}}^{\prime} & =\sum_{n=1}^{\infty} \mathbf{A}_{\tilde{2}}^{\prime(n)}, & \mathbf{A}_{L, \tilde{2}} & =\sum_{n=1}^{\infty} \mathbf{A}_{L, \tilde{2}}^{(n)} .
\end{aligned}
$$

Note that the last three sums begin at $n=1$, in contrast to the sum for $\mathbf{A}_{L, 3}^{(u)}$, Eq. (53), which begins at $n=0$. It is because of this that $\boldsymbol{\sigma}^{\dagger *}$ must be subtracted from $\mathbf{A}_{L, 3}^{(u)}$ in the final result, Eq. (55).

\section{Decompositions of $A_{L, 3}^{\prime(u)}, A_{L, 3}^{(u)}$ and $A_{L, \tilde{2}}$}

In this subsection, we continue the decomposition of the quantities entering $C_{L}^{\left[B_{2}\right]}$, Eq. (49), by considering the finitevolume end caps $\mathbf{A}_{L, 3}^{\prime(u)}$ and $\mathbf{A}_{L, 3}^{(u)}$. In addition, we decompose the related quantity $\mathbb{A}_{L, \tilde{2}}$ that appears in Eq. (55). The results we obtain are

$$
\begin{aligned}
\mathbf{A}_{L, 3}^{\prime(u)}= & \mathbf{A}_{3}^{\prime(u)}+\left(2 \mathbf{A}_{3}^{\prime(s)} \mathbf{F}+\mathbf{A}_{\tilde{2}}^{\prime} \mathbf{G}_{\rho} \boldsymbol{\Gamma} \mathbf{G}\right)\left(\mathbf{K}_{L, 33}^{(u, u)}+\mathbf{K}_{2}\right) \\
& +\mathbf{A}_{\tilde{2}}^{\prime}\left(\mathbf{F}_{\rho \pi} \mathbf{K}_{L, \tilde{2} 3}^{(u)}+\mathbf{G}_{\rho} \boldsymbol{\Gamma}\right) \\
\mathbf{A}_{L, 3}^{(u)}= & \mathbf{A}_{3}^{(u)}+\left(\mathbf{K}_{L, 33}^{(u, u)}+\mathbf{K}_{2}\right)\left(\mathbf{F} 2 \mathbf{A}_{3}^{(s)}+\mathbf{G} \boldsymbol{\Gamma} \overline{\mathbf{G}}_{\rho} \mathbf{A}_{\tilde{2}}\right) \\
& +\left(\mathbf{K}_{L, 3 \tilde{2}}^{(u)} \mathbf{F}_{\rho \pi}+\boldsymbol{\Gamma} \overline{\mathbf{G}}_{\rho}\right) \mathbf{A}_{\tilde{2}} \\
\mathbf{A}_{L, \tilde{2}}= & \mathbf{A}_{\tilde{2}}+\mathbf{K}_{L, \tilde{2} 3}^{(u)}\left(\mathbf{F} 2 \mathbf{A}_{3}^{(s)}+\mathbf{G \Gamma} \overline{\mathbf{G}}_{\rho} \mathbf{A}_{\tilde{2}}\right)+\mathbf{K}_{L, \tilde{2} \tilde{2}} \mathbf{F}_{\rho \pi} \mathbf{A}_{\tilde{2}} .
\end{aligned}
$$

Here we have introduced three new finite-volume K matrices,

$$
\begin{aligned}
\mathbf{K}_{L, \tilde{2} \tilde{2}} & =\sum_{n=2}^{\infty} \mathbf{K}_{L, \tilde{2} \tilde{2}}^{(n)}, \quad \mathbf{K}_{L, \tilde{2} 3}^{(u)} \equiv \sum_{n=2}^{\infty} \mathbf{K}_{L, \tilde{2} 3}^{(n, u)}, \\
\mathbf{K}_{L, 3 \tilde{2}}^{(u)} & \equiv \sum_{n=2}^{\infty} \mathbf{K}_{L, 3 \tilde{2}}^{(n, u)},
\end{aligned}
$$

all closely related to $\mathbf{K}_{L, 33}^{(u, u)}$. Specifically, $\mathbf{K}_{L, \tilde{2} \tilde{2}}^{(n)}$ is obtained from $\mathbf{K}_{L, 33}^{(n, u, u)}$ by replacing the $\mathcal{K}_{2}$ s on both ends with factors of $\boldsymbol{\Gamma}$, and connecting these $\boldsymbol{\Gamma}$ s to the adjacent $\mathcal{K}_{2}$ s with the smooth (G-subtracted) part of the neighboring exchange propagator. This is shown in Fig. 2(d). $\mathbf{K}_{L, \tilde{2} 3}^{(n, u)}$ and $\mathbf{K}_{L, 3 \tilde{2}}^{(n, u)}$ are obtained by performing these steps on only one side of $\mathbf{K}_{L, 33}^{(n, u, u)}$ while leaving the other side unchanged, as shown in Figs. 2(b) and 2(c), respectively. Also new in Eqs. (58)-(60) are the infinite-volume end caps $\mathbf{A}_{3}^{\prime(u)}, \mathbf{A}_{3}^{(u)}, \mathbf{A}_{\tilde{2}}$. and $\mathbf{A}_{\tilde{2}}$. These are defined below.

Intuitively, Eqs. (58)-(60) summarize the various ways that finite-volume effects enter the end cap functions. For example, Eq. (58) splits $\mathbf{A}_{L, 3}^{\prime(u)}$ into its infinite-volume counterpart, $\mathbf{A}_{3}^{\prime(u)}$, plus six types of finite-volume corrections (counted by multiplying out the products of binomials in the middle term). Each term is characterized by a different type of cut factor; e.g., $\mathbf{F}$ encodes the finitevolume effects associated with two of the three particles 
(a)

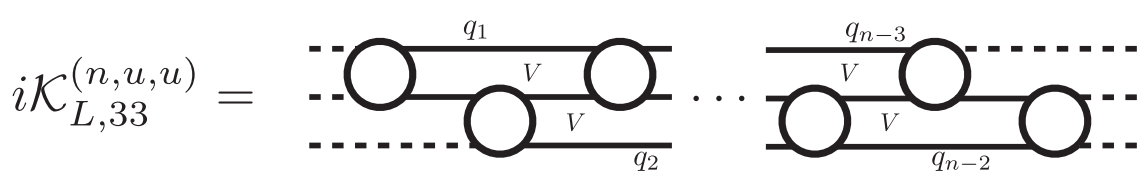

(b)
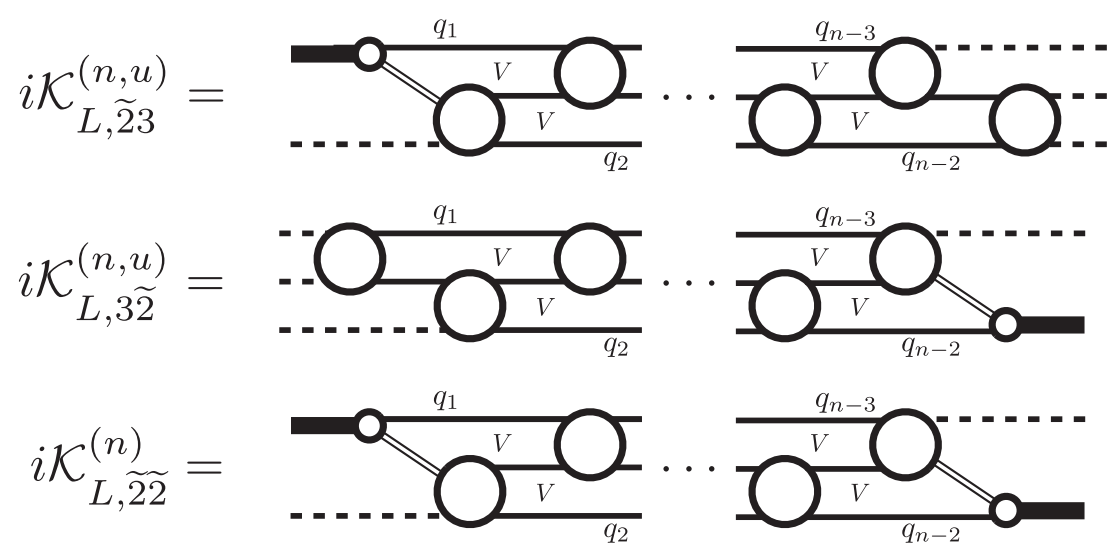

FIG. 2. Diagrammatic definitions of the elements of the finite-volume $\mathrm{K}$ matrices involving three-particle or $\rho \pi$ external states. The notation is as in Fig. 1.

propagating between adjacent pairwise rescatterings and $\mathbf{G}$ describes the volume effects of an exchanged particle. Similarly, $\mathbf{G}_{\rho}$ and $\mathbf{F}_{\rho \pi}$ correspond to different types of volume effects associated with the K-matrix poles. $\mathbf{A}_{L, 3}^{(u)}$, in Eq. (59), is simply a mirror image of $\mathbf{A}_{L, 3}^{\prime(u)}$, while $\mathbf{A}_{L, \tilde{2}}$, in Eq. (60), is given by replacing the rightmost state with a $\tilde{2}$ and dropping terms that do not arise with this type of external state.

To derive these results we use a similar method to that of the previous subsection. We first consider $\mathbf{A}_{L, 3}^{\prime(u)}$, and introduce $\mathbf{A}_{L, 3}^{\prime(m, n, u)}$, which contains $n$ two-particle kernels, $m$ integrated loop momenta and $n-m$ summed loop momenta. As for $C_{L, 0 F}^{(m, n)}$, the integrated $\mathrm{K}$ matrices are $i \tilde{\mathcal{K}}_{2}^{\prime} \mathrm{s}$, while the summed ones are the full $i \mathcal{K}_{2} \mathrm{~s}$. We need this quantity for $n \geq m \geq 1$ together with the special case $n=m=0$, giving $\mathbf{A}_{L, 3}^{\prime(0,0, u)}=\mathbf{A}_{L, 3}^{\prime(0, u)}=\boldsymbol{\sigma}^{*}$. A second special case is $\mathbf{A}_{L, 3}^{\prime(1, n, u)}=\mathbf{A}_{L, 3}^{\prime(n, u)}$ (since there is always one integrated loop for $n \geq 1$ ). Finally, we note that the fully integrated version is an infinite-volume quantity, $\mathbf{A}_{L, 3}^{\prime(n, n, u)}=\mathbf{A}_{3}^{\prime(n, u)}$. This quantity is shown diagrammatically in Fig. 1(d), and differs from the quantity $\mathbf{A}^{\prime(n, s)}$ encountered above only by the choice of spectator propagator.

The steps detailed in Appendix B 1 apply also here, except that the right end caps $\boldsymbol{\sigma}^{\dagger *}$ are replaced with an onshell three-particle state. We find that the resulting recursion equations are

$$
\mathbf{A}_{L, 3}^{\prime(m+1, n, u)}= \begin{cases}\mathbf{A}_{L, 3}^{\prime(m+2, n, u)}+\left(2 \mathbf{A}_{3}^{\prime(m+1, s)} \mathbf{F}+\mathbf{A}_{\tilde{2}}^{\prime(m+1)} \mathbf{G}_{\rho} \mathbf{\Gamma} \mathbf{G}\right) \mathbf{K}_{L, 33}^{(n-m-1, u, u)}+\mathbf{A}_{\tilde{2}}^{\prime(m+1)} \mathbf{F}_{\rho \pi} \mathbf{K}_{L, \tilde{2} 3}^{(n-m, u)} & 0 \leq m<n-2, \\ \mathbf{A}_{L, 3}^{\prime(n, n, u)}+\left(2 \mathbf{A}_{3}^{\prime(n-1, s)} \mathbf{F}+\mathbf{A}_{\tilde{2}}^{\prime(n-1)} \mathbf{G}_{\rho} \boldsymbol{\Gamma} \mathbf{G}\right) \mathbf{K}_{2}+\mathbf{A}_{\tilde{2}}^{\prime(n-1)} \mathbf{F}_{\rho \pi} \mathbf{K}_{L, \tilde{2} 3}^{(2, u)} & 0 \leq m=n-2, \\ \mathbf{A}_{3}^{\prime(n, u)}+\mathbf{A}_{\tilde{2}}^{\prime(n)} \mathbf{G}_{\rho} \boldsymbol{\Gamma} & 0 \leq m=n-1 .\end{cases}
$$

We stress that all quantities to the left of the cuts are, by construction, identical to those appearing in Eq. (56). The quantities appearing to the right, however, have changed: $\mathbf{A}_{L, 3}^{(n, u)}$ has been replaced by $\mathbf{K}_{L, 33}^{(n, u, u)}$ and $\mathbf{A}_{L, \tilde{2}}^{(n)}$ has been replaced by $\mathbf{K}_{L, 23}^{(n, u)}$.

Solving the recursion relation for $\mathbf{A}_{L, 3}^{\prime(n, u)}$ and summing over $n$ using the definition

$$
\mathbf{A}_{3}^{\prime(u)} \equiv \sum_{n=0}^{\infty} \mathbf{A}_{3}^{\prime(n, u)}
$$

yields Eq. (58). We observe that the combination $\mathbf{K}_{L, 33}^{(u, u)}+$ $\mathbf{K}_{2}$ appears. This arises because the sum over $n$ for $\mathbf{K}_{L, 33}^{(u, u)}$ begins at $n=2$, since at least two factors of $i \mathcal{K}_{2}$ are needed for a connected scattering of three particles. The $n=1$ term then becomes simply $\mathbf{K}_{2}$. Similarly, the $n=1$ term is absent in the definition of $\mathbf{F}_{\rho \pi} \mathbf{K}_{L, \tilde{2} 3}^{(u)}$ and this leads to the additional contribution containing $\mathbf{G}_{\rho} \boldsymbol{\Gamma}$. Note that, if $\mathbf{G}_{\rho}$ and $\mathbf{F}_{\rho \pi}$ are set to 0 , then we recover the result given in Eq. (186) of Ref. [5]. 
The horizontal reflection of Eq. (58) gives the decomposition of the other end cap, Eq. (59).

Finally, we need to decompose $\mathbb{A}_{L, \tilde{2}}$. We recall that this is the finite-volume right end cap, defined diagrammatically in Fig. 1(f). It thus differs from $\mathbf{A}_{L, 3}$ only in its final state, in which a factor of $\boldsymbol{\Gamma}$ combines with the smooth part of the exchange propagator. This means that we can adapt the result from that for $\mathbf{A}_{L, 3}$ by replacing the three-particle external state with a two-particle one, and dropping the contribution from the $\mathbf{K}_{2}$ factor on the end (since this is replaced by smooth quantities). The result is given in Eq. (60).

\section{E. Decomposition of $K_{L, \tilde{2} \tilde{2},}, K_{L, \tilde{2} 3}^{(u)}, K_{L, 3 \tilde{2} \tilde{2}}^{(u)}$ and $K_{L, 33}^{(u, u)}$}

In this subsection we complete the decomposition of the quantities entering $C_{L}^{\left[B_{2}\right]}$ into infinite-volume objects and finite-volume cuts, with some technical details relegated to Appendix B 2.

What remains is to decompose the four finite-volume $\mathrm{K}$ matrices whose components are shown in Fig. 2. They are conveniently packaged into a two-by-two matrix

$$
\mathbf{K}_{L}^{(u)} \equiv\left(\begin{array}{cc}
\mathbf{K}_{L, \tilde{2} \tilde{2}} & \mathbf{K}_{L, \tilde{2} 3}^{(u)} \\
\mathbf{K}_{L, 3 \tilde{2}}^{(u)} & \mathbf{K}_{L, 33}^{(u, u)}
\end{array}\right) .
$$

The result we derive in the following can be written compactly as

$\mathbf{K}_{L}^{(u)}=\left(\begin{array}{cc}0 & 0 \\ 0 & \mathbf{K}_{L, 33}^{(0)}\end{array}\right)+\mathcal{E}_{L} \overline{\mathbf{V}} \mathbf{K}_{\mathrm{df}}^{(u)} \frac{1}{1-\mathbf{X} \mathbf{K}_{\mathrm{df}}^{(u)}} \mathbf{V} \mathcal{E}_{R}$,

where

$$
\begin{gathered}
\mathbf{K}_{L, 33}^{(0)} \equiv \mathbf{K}_{2} \mathbf{G}_{\mathbf{K}} \mathbf{K}_{2}=\frac{1}{1-\mathbf{K}_{2} \mathbf{G}} \mathbf{K}_{2} \mathbf{G K}_{2}, \\
\mathbf{X} \equiv\left(\begin{array}{cc}
\mathbf{F}_{\rho \pi}+\mathbf{G}_{\rho} \boldsymbol{\Gamma} \mathbf{G}_{\mathbf{K}} \boldsymbol{\Gamma} \overline{\mathbf{G}}_{\rho} & \mathbf{G}_{\rho} \boldsymbol{\Gamma} \mathbf{G}_{\mathbf{K}} \\
\mathbf{G}_{\mathbf{K}} \boldsymbol{\Gamma} \overline{\mathbf{G}}_{\rho} & \mathbf{G}_{\mathbf{K}}
\end{array}\right), \\
\mathbf{V} \equiv\left(\begin{array}{cc}
1 & \mathbf{G}_{\rho} \boldsymbol{\Gamma} \\
0 & 1
\end{array}\right), \quad \overline{\mathbf{V}} \equiv\left(\begin{array}{cc}
1 & 0 \\
\boldsymbol{\Gamma} \overline{\mathbf{G}}_{\rho} & 1
\end{array}\right), \\
\mathcal{E}_{L} \equiv\left(\begin{array}{ccc}
1 & 0 & \\
0 & 1+\mathbf{T G}
\end{array}\right), \quad \mathcal{E}_{R} \equiv\left(\begin{array}{cc}
1 & 0 \\
0 & 1+\mathbf{G T}
\end{array}\right), \\
\mathbf{G}_{\mathbf{K}} \equiv \frac{1}{1-\mathbf{G K}_{2}} \mathbf{G}, \quad \mathbf{T} \equiv \mathbf{K}_{2} \frac{1}{1-\mathbf{G K}_{2}} .
\end{gathered}
$$

The final new quantity is $\mathbf{K}_{\mathrm{df}}^{(u)}$. This is a two-by-two matrix of infinite-volume, divergence-free $\mathrm{K}$ matrices, defined below in Eq. (80). The motivation for all these new quantities is described in more detail during the following derivation.

As above, our task is to replace all summed loop momenta with integrals, separating out the divergences due to both the three-particle on-shell intermediate states and the poles in $\mathcal{K}_{2}$. It turns out that, at first, we do not need to decompose those factors of $\mathcal{K}_{2}$ that lie directly adjacent to $\mathbf{K}_{L}^{(u)}$ components with a 3 index. This applies, for example, to the $\mathcal{K}_{2} \mathrm{~S}$ at both the left and right ends of $\mathbf{K}_{L, 33}^{(n, u, u)}$ in Fig. 2. These can remain as the full $\mathrm{K}$ matrices, despite containing poles, since they do not appear in sums. Leaving these factors of $\mathcal{K}_{2}$ unseparated leads to shorter expressions at intermediate stages, at the cost of requiring an additional step to remove the final divergences. We denote by $\mathbf{K}_{33}, \mathbf{K}_{32}$ and $\mathbf{K}_{\tilde{2} 3}$ these intermediate infinite-volume quantities that still contain external divergences from the external $\mathcal{K}_{2}$.

The method we use here is simpler than the approach adopted in Ref. [5], where the result for any number of $\mathcal{K}_{2}$ factors was deduced by working out the cases with 2,3 , and 4 factors of $\mathcal{K}_{2}$ and then determining the pattern. Here we use matrix equations that take care of all orders at once. We find it convenient to keep track of finite-volume contributions in two stages: first those from $\mathbf{G}$ cuts and second those from K-matrix poles, the latter leading to $\mathbf{G}_{\rho}$ and $\mathbf{F}_{\rho \pi}$ cuts.

We begin by considering $\mathbf{K}_{L, 33}^{(u, u)}$. Moving from left to right, we consider each three-particle intermediate state in turn. At each stage this consists of two fully dressed propagators, e.g., $\Delta(a) \Delta(b)$ with $a$ being the spectator momentum. We replace this with the product $2 \pi \delta\left(a^{0}-\right.$ $\left.\omega_{a}\right)(2 \omega) \mathbf{G} L^{6}$ together with the difference, which is a smooth function of $\vec{a}$. By construction, the insertion of G sets the nonspectator pairs on either side on shell. The details of how this works are unchanged from Ref. [5] and we do not repeat them here. After the substitution is made, in the term containing the factor of $\mathbf{G}$ this first stage of decomposition is complete and a factor of $\mathbf{K}_{L, 33}^{(u, u)}$ appears to the right of $\mathbf{G}$. In the term containing the smooth residue we proceed to the next intermediate state to the right and repeat the decomposition. See Fig. 3 for a diagrammatic sketch of the first steps in this procedure.

This procedure leads to the equation

$$
\begin{aligned}
\mathbf{K}_{L, 33}^{(u, u)}= & \mathbf{K}_{2} \mathbf{G K}_{2}+\mathbf{K}_{L, 33}\left(1+\mathbf{G K}_{2}\right) \\
& +\left(\mathbf{K}_{L, 33}+\mathbf{K}_{2}\right) \mathbf{G} \mathbf{K}_{L, 33}^{(u, u)},
\end{aligned}
$$

where $\mathbf{K}_{L, 33}$ is the same as $\mathbf{K}_{L, 33}$ except that all intermediate states have propagators replaced by the smooth difference described above. For brevity, we have dropped the " $u$ " superscripts on $\mathbf{K}_{L, 33}$. We note also that the terms involving $\mathbf{K}_{2}$ in this result arise from special cases where, after the insertion of $\mathbf{G}$, there is only a single $\mathbf{K}_{2}$ on one or both sides.

If there were no poles in $\mathcal{K}_{2}$ we could replace the momentum sums in $\mathbf{K}_{L, 33}$ with integrals and obtain the divergence-free $\mathrm{K}$ matrix. This was the procedure followed in Ref. [5]. However, here we need to extract the finitevolume effects that arise from the K-matrix poles. To do so, we work through $\mathbf{K}_{L, 33}$ from left to right, replacing each full $\mathcal{K}_{2}$ with the $\mathbf{F}_{\rho \pi}$ cut and the difference, with the latter 


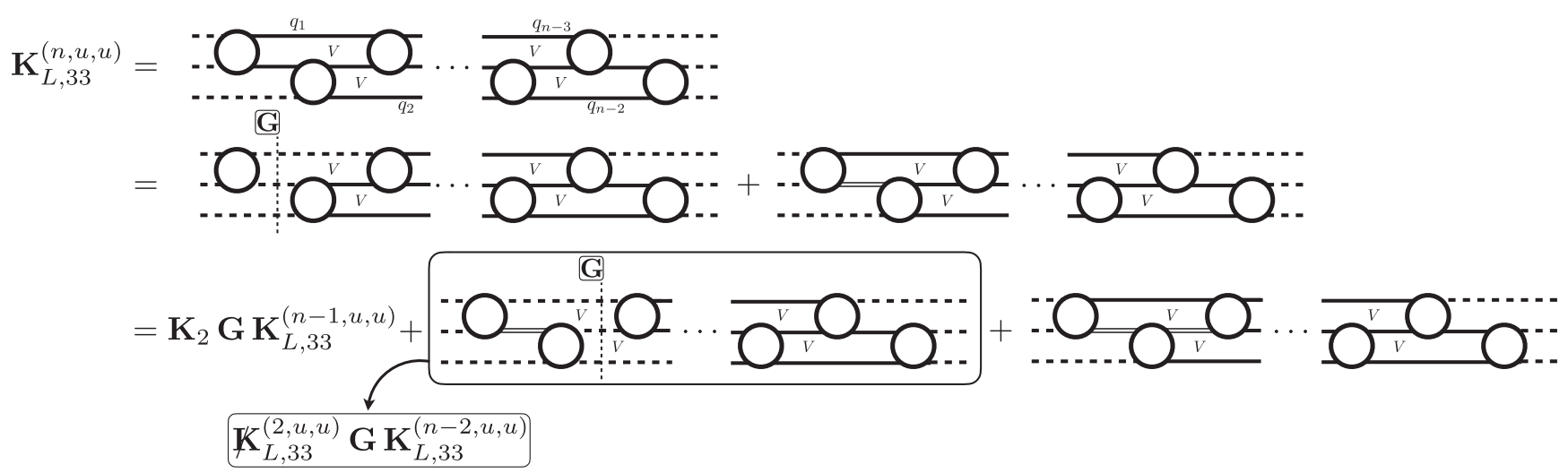

FIG. 3. Diagrammatic description of the procedure described in the text leading to Eq. (71). The notation is the same as in Fig. 1, with the addition of a dashed line to indicate a propagator proportional to $\mathbf{G}$ (if internal) or an amputated propagator (if external).

being a smooth function of the spectator momentum. In the term with the $\mathbf{F}_{\rho \pi}$ cut the procedure stops, leaving a factor of $\mathbf{K}_{L, \tilde{2} 3}$ to the right. The remaining, $\mathbf{F}_{\rho \pi}$-independent terms build up quantities in which all loop sums can be replaced by integrals because the integrands are divergence free. These are the quantities mentioned above that contain divergences only in the external $\mathrm{K}$ matrices and are denoted by $\mathbf{K}_{33}, \mathbf{K}_{32}$ etc. [Again we drop the superscripts $(u)$ for brevity.] The result is

$$
\mathbf{K}_{L, 33}=\mathbf{K}_{33}+\mathbf{K}_{32} \mathbf{F}_{\rho \pi} \mathbf{K}_{L, \tilde{2} 3} .
$$

Proceeding in the same way for $\mathbf{K}_{L, \tilde{2} 3}^{(u)}$ and $\mathbf{K}_{L, \tilde{2} 3}$ we obtain

$$
\begin{gathered}
\mathbf{K}_{L, \tilde{2} 3}^{(u)}=\mathbf{K}_{L, \tilde{2} 3}\left(1+\mathbf{G K}_{2}+\mathbf{G} \mathbf{K}_{L, 33}^{(u, u)}\right), \\
\mathbf{K}_{L, \tilde{2} 3}=\mathbf{K}_{\tilde{2} 3}+\mathbf{K}_{\tilde{2} \tilde{2}} \mathbf{F}_{\rho \pi} \mathbf{K}_{L, \tilde{2} 3} .
\end{gathered}
$$

We note here the appearance of $\mathbf{K}_{\tilde{2} \tilde{2}}$, which is the infinitevolume version of $\mathbf{K}_{L, \tilde{2} \tilde{2}}$ once all divergences have been removed. This quantity does not have factors of $\mathcal{K}_{2}$ at its ends, so it is already divergence free. These matrix equations can now be solved sequentially. The solution to Eq. (74) is

$$
\mathbf{K}_{L, \tilde{2} 3}=\frac{1}{1-\mathbf{K}_{\tilde{2} \tilde{2}} \mathbf{F}_{\rho \pi}} \mathbf{K}_{\tilde{2} 3},
$$

and inserting this in Eq. (72) yields

$$
\mathbf{K}_{L, 33}=\mathbf{K}_{33}+\mathbf{K}_{3 \tilde{2}} \mathbf{F}_{\rho \pi} \frac{1}{1-\mathbf{K}_{\tilde{2} \tilde{2}} \mathbf{F}_{\rho \pi}} \mathbf{K}_{\tilde{2} 3} .
$$

Taken together, Eqs. (71) and (76) give a complete prescription for writing $\mathbf{K}_{L, 33}^{(u, u)}$ in terms of infinite-volume quantities and finite-volume cuts. In Appendix B 2 we outline the remaining steps in this decomposition explicitly. In the Appendix we also work through the decompositions for the remaining finite-volume $\mathrm{K}$ matrices, $\mathbf{K}_{L, 3 \tilde{2}}^{(u)}$ and $\mathbf{K}_{L, \tilde{2} \tilde{2}}$, and for the slashed objects, $\mathbf{K}_{L, 3 \tilde{2}}$ and $\mathbf{K}_{L, \tilde{2} \tilde{2}}$.
The procedure in all cases is similar to that outlined above: One works through the summed loops in a diagram from left to right, substituting singular and smooth pieces for the propagators to reach matrix equations for the various finitevolume objects entering the correlator. We find that the solutions to the resulting equations can be succinctly displayed in two key relations,

$$
\begin{gathered}
\mathbf{K}_{L}^{(u)}=\left(\begin{array}{cc}
0 & 0 \\
0 & \mathbf{K}_{L, 33}^{(0)}
\end{array}\right)+\mathcal{E}_{L} \frac{1}{\mathbf{K}_{L}^{-1}-\mathcal{G}_{\mathbf{K}}} \mathcal{E}_{R}, \\
\mathbf{K}_{L}^{-1}=\mathcal{K}^{-1}-\mathcal{F}_{\rho \pi},
\end{gathered}
$$

where we have introduced two-by-two matrix generalizations of the various quantities appearing above,

$$
\begin{aligned}
& \mathbf{K}_{L} \equiv\left(\begin{array}{cc}
\mathbf{K}_{L, \tilde{2} \tilde{2}} & \mathbf{K}_{L, \tilde{2} 3} \\
\mathbf{K}_{L, 3 \tilde{2}} & \mathbf{K}_{L, 33}
\end{array}\right), \quad \mathcal{K} \equiv\left(\begin{array}{cc}
\mathbf{K}_{\tilde{2} \tilde{2}} & \mathbf{K}_{\tilde{2} 3} \\
\mathbf{K}_{3 \tilde{2}} & \mathbf{K}_{33}
\end{array}\right), \\
& \mathcal{F}_{\rho \pi} \equiv\left(\begin{array}{cc}
\mathbf{F}_{\rho \pi} & 0 \\
0 & 0
\end{array}\right), \quad \text { and } \quad \mathcal{G}_{\mathbf{K}} \equiv\left(\begin{array}{cc}
0 & 0 \\
0 & \mathbf{G}_{\mathbf{K}}
\end{array}\right) .
\end{aligned}
$$

To complete the work of this section we need to remove the $\mathrm{K}$-matrix poles contained in $\mathcal{K}$. This is necessary in order to symmetrize over choices of spectator, as we see in the next subsection. To do so we introduce appropriate factors of $\mathbf{G}_{\rho}$ corresponding to the poles in the external factors of $\mathcal{K}_{2}$. This leads to the result

$$
\mathcal{K}=\overline{\mathbf{V}} \mathbf{K}_{\mathrm{df}}^{(u)} \mathbf{V},
$$

where $\overline{\mathbf{V}}$ and $\mathbf{V}$ are defined in Eq. (67). This relation defines a matrix of nonsingular infinite-volume $\mathrm{K}$ matrices already displayed in the result given at the beginning of the subsection, ${ }^{13}$

\footnotetext{
${ }^{13}$ We note that $\mathbf{K}_{\mathrm{df}, \tilde{2} \tilde{2}}=\mathbf{K}_{\tilde{2} \tilde{2}}$, so the df subscript is not needed for this component. We include it anyway for uniformity of notation.
} 


$$
\mathbf{K}_{\mathrm{df}}^{(u)} \equiv\left(\begin{array}{ll}
\mathbf{K}_{\mathrm{df}, \tilde{2} \tilde{2}} & \mathbf{K}_{\mathrm{df}, \tilde{2} 3}^{(u)} \\
\mathbf{K}_{\mathrm{df}, 3 \tilde{2}}^{(u)} & \mathbf{K}_{\mathrm{df}, 33}^{(u, u)}
\end{array}\right) .
$$

Combining Eqs. (77), (78) and (80) we reach the main result of this subsection given in Eq. (65) above. The quantity $\mathbf{X}$ appears as

$$
\mathbf{X} \equiv \mathbf{V}\left(\mathcal{F}_{\rho \pi}+\mathcal{G}_{\mathbf{K}}\right) \overline{\mathbf{V}}
$$

which can be rearranged into the form shown in Eq. (67).

At this stage we have decomposed all objects appearing in $C_{L}^{\left[B_{2}\right]}$ into matrix products of finite- and infinite-volume quantities. In the following subsections we reshuffle these decompositions into a compact form for this partial finitevolume correlator. We then show how the three-particle Bethe-Salpeter kernls, $B_{3}$, can be reintroduced to derive the main result of the section, Eq. (38).

\section{F. $\left(\mathbf{K}_{\mathrm{df}}\right)^{0}$ contribution to $C_{L}^{\left[\boldsymbol{B}_{2}\right]}$}

We now have all the ingredients needed to determine the volume dependence of the correlator $C_{L}^{\left[B_{2}\right]}$. The initial decomposition of this object is given in Eq. (49). To derive the final form we work order by order in $\mathbf{K}_{\mathrm{df}}$, and begin by considering the contributions that are independent of this local three-body interaction. In particular, in this subsection we demonstrate

$$
\begin{aligned}
C_{L}^{\left[B_{2}\right]}-C_{\infty}^{\left[B_{2}\right]}-\delta C_{\infty}^{\left[B_{2}\right]}= & \left(\begin{array}{ll}
\mathbf{A}_{\tilde{2}}^{\prime} & \mathbf{A}_{3}^{\prime}
\end{array}\right)\left(\begin{array}{ll}
\mathbf{F}_{\tilde{2} \tilde{2}} & \mathbf{F}_{\tilde{2} 3} \\
\mathbf{F}_{3 \tilde{2}} & \mathbf{F}_{33}
\end{array}\right)\left(\begin{array}{c}
\mathbf{A}_{\tilde{2}} \\
\mathbf{A}_{3}
\end{array}\right) \\
& +\mathcal{O}\left(\mathbf{K}_{\mathrm{df}}\right),
\end{aligned}
$$

where $\mathbf{F}_{33}, \mathbf{F}_{\tilde{2} \tilde{2}}, \mathbf{F}_{\tilde{2} 3}$, and $\mathbf{F}_{3 \tilde{2}}$ are defined in Eqs. (45)-(48), respectively, while $\delta C_{\infty}^{\left[B_{2}\right]}$ is an additional volumeindependent term, defined at leading order in $\mathbf{K}_{\mathrm{df}}$ in Eq. (94) below. As mentioned in the introduction, many of the steps in the derivation of Eq. (83) presented here have been checked using a Mathematica notebook implementing the package The NCAlgebra Suite [33]. Equations verified in this way are preceded by the indicator " $(\checkmark N C A \checkmark)$."

If $\mathbf{K}_{\mathrm{df}}^{(u)}=0$, the only nonzero component of $\mathbf{K}_{L}^{(u)}$ is $\mathbf{K}_{L, 33}^{(u, u)}$, which becomes $\mathbf{K}_{L, 33}^{(0)}$, defined in Eq. (66). Thus the infinite sum in Eq. (49) becomes $(\checkmark N C A \checkmark)$

$$
\begin{aligned}
\mathbf{F}_{33}^{(0)} \sum_{n=0}^{\infty}\left(\mathbf{K}_{L, 33}^{(u, u)} \mathbf{F}_{33}^{(0)}\right)^{n} \rightarrow \mathbf{Z} & \equiv \mathbf{F}_{33}^{(0)} \sum_{n=0}^{\infty}\left(\mathbf{K}_{L, 33}^{(0)} \mathbf{F}_{33}^{(0)}\right)^{n} \\
& =\mathbf{F} \frac{1}{1-\mathbf{T F}},
\end{aligned}
$$

where the arrow indicates $\mathbf{K}_{\mathrm{df}}^{(u)} \rightarrow 0$. Here we have used $\mathbf{F}_{33}^{(0)}$ and $\mathbf{T}$, defined in Eqs. (50) and (70) respectively. In this same limit the quantities $\mathbf{A}_{L, 3}^{(u)}, \mathbf{A}_{L, 3}^{(u)}$ and $\mathbf{A}_{L, \tilde{2}}$ simplify to $(\checkmark N C A \checkmark)$,

$$
\mathbf{A}_{L, 3}^{\prime(u)} \rightarrow \mathbf{A}_{L, 3}^{\prime(u),\{0\}}=\mathbf{A}_{3}^{\prime(u)}+2 \mathbf{A}_{3}^{\prime(s)} \mathbf{F T}+\mathbf{A}_{\tilde{2}}^{\prime} \mathbf{G}_{\rho} \boldsymbol{\Gamma}(1+\mathbf{G T}),
$$

$$
\mathbf{A}_{L, 3}^{(u)} \rightarrow \mathbf{A}_{L, 3}^{(u),\{0\}}=\mathbf{A}_{3}^{(u)}+\mathbf{T F} 2 \mathbf{A}_{3}^{(s)}+(1+\mathbf{T G}) \mathbf{\Gamma} \overline{\mathbf{G}}_{\rho} \mathbf{A}_{\tilde{2}},
$$

$$
\mathbf{A}_{L, \tilde{2}} \rightarrow \mathbf{A}_{\tilde{2}},
$$

where the superscript $\{n\}$ indicates the contribution to the indicated object with $n$ factors of $\mathbf{K}_{\mathrm{df}}$.

At this stage we can use the following result from Ref. [5]: if $2 \mathbf{A}_{3}^{(s)}$ is adjacent to a factor of $\mathbf{F K}_{2}$ then it can be replaced by $\mathbf{A}^{\prime(s)}+\mathbf{A}^{\prime(\tilde{s})}$, with $(\tilde{s})$ indicating the third independent permutation of the external momenta. This is the case in Eq. (85) because $\mathbf{T}$ always has a factor $\mathbf{K}_{2}$ on its left-hand end. The same holds for the factor of $2 \mathbf{A}_{3}^{\prime(s)}$ in Eq. (55), because $\mathbf{A}_{L, 3}^{(u)}$ and $\boldsymbol{\sigma}^{\dagger *}$ are symmetric under the interchange of the nonspectator pair. Similarly, the factor of $2 \mathbf{A}_{3}^{(s)}$ in Eq. (86) can be replaced by $\mathbf{A}_{3}^{(s)}+\mathbf{A}_{3}^{(\tilde{s})}$. These substitutions are important because the fully symmetrized end caps are given by

$\mathbf{A}_{3}^{\prime} \equiv \mathbf{A}_{3}^{\prime(u)}+\mathbf{A}_{3}^{\prime(s)}+\mathbf{A}_{3}^{\prime(\tilde{s})}$, and $\quad \mathbf{A}_{3} \equiv \mathbf{A}_{3}^{(u)}+\mathbf{A}_{3}^{(s)}+\mathbf{A}_{3}^{(\tilde{s})}$.

We expect the final result to depend only on symmetrized quantities. In the following, for the sake of brevity, we use $2 \mathbf{A}^{(s)}$ as an abbreviation for $\mathbf{A}^{(s)}+\mathbf{A}^{(\tilde{s})}$ and $2 \mathbf{A}^{\prime(s)}$ for $\mathbf{A}^{\prime(s)}+\mathbf{A}^{\prime(\tilde{s})}$. Using this simplification, we can rewrite Eqs. (85) and (86) as

$\mathbf{A}_{L, 3}^{\prime(u),\{0\}}=\mathbf{A}_{3}^{\prime}-2 \mathbf{A}_{3}^{\prime(s)}(1-\mathbf{F T})+\mathbf{A}_{2}^{\prime} \mathbf{G}_{\rho} \boldsymbol{\Gamma}(1+\mathbf{G T})$,

$\mathbf{A}_{L, 3}^{(u),\{0\}}=\mathbf{A}_{3}-(1-\mathbf{T F}) 2 \mathbf{A}_{3}^{(s)}+(1+\mathbf{T G}) \mathbf{\Gamma} \overline{\mathbf{G}}_{\rho} \mathbf{A}_{\tilde{2}}$.

The final quantity we are missing is $C_{L, 0 F}$, whose decomposition is given in Eq. (55). Sending $\mathbf{K}_{\mathrm{df}} \rightarrow 0$ in this result, and using Eq. (90), gives $(\checkmark N C A \checkmark)$,

$$
\begin{aligned}
C_{L, 0 F} \rightarrow C_{L, 0 F}^{\{0\}}= & C_{\infty}^{\left[B_{2}\right],\{0\}}+\left(2 \mathbf{A}_{3}^{\prime(s)} \mathbf{F}+\mathbf{A}_{\tilde{2}}^{\prime} \mathbf{G}_{\rho} \mathbf{\Gamma} \mathbf{G}\right) \\
& \times\left(\mathbf{A}_{3}-(1-\mathbf{T F}) 2 \mathbf{A}_{3}^{(s)}\right. \\
& \left.+(1+\mathbf{T G}) \mathbf{\Gamma} \overline{\mathbf{G}}_{\rho} \mathbf{A}_{\tilde{2}}-\boldsymbol{\sigma}^{\dagger *}\right)+\mathbf{A}_{\tilde{2}}^{\prime} \mathbf{F}_{\rho \pi} \mathbf{A}_{\tilde{2}} .
\end{aligned}
$$

We have now gathered all the pieces to evaluate the full correlation function, decomposed in Eq. (49), at $\mathcal{O}\left(\left[\mathbf{K}_{\mathrm{df}}\right]^{0}\right)$. This equation reduces to 


$$
C_{L}^{\left[B_{2}\right],\{0\}}=C_{L, 0 F}^{\{0\}}-\frac{2}{3} \boldsymbol{\sigma}^{*} \mathbf{F} \boldsymbol{\sigma}^{\dagger *}+\mathbf{A}_{L, 3}^{\prime(u),\{0\}} \mathbf{Z} \mathbf{A}_{L, 3}^{(u),\{0\}} .
$$

Substituting Eqs. (84), (89) and (90) and significantly rearranging, we find $(\boldsymbol{\checkmark} N C A \checkmark)$,

$$
\begin{aligned}
C_{L}^{\left[B_{2}\right],\{0\}}= & C_{\infty}^{\left[B_{2}\right],\{0\}}+\delta C_{\infty}^{\left[B_{2}\right],\{0\}} \\
& +\left(\begin{array}{ll}
\mathbf{A}_{\tilde{2}}^{\prime} & \mathbf{A}_{3}^{\prime}
\end{array}\right)\left(\begin{array}{ll}
\mathbf{F}_{\tilde{2} \tilde{2}} & \mathbf{F}_{\tilde{2} 3} \\
\mathbf{F}_{3 \tilde{2}} & \mathbf{F}_{33}
\end{array}\right)\left(\begin{array}{c}
\mathbf{A}_{\tilde{2}} \\
\mathbf{A}_{3}
\end{array}\right),
\end{aligned}
$$

where

$$
\begin{aligned}
\delta C_{\infty}^{\left[B_{2}\right],\{0\}} \equiv & -\frac{2}{3} \boldsymbol{\sigma}^{*} \mathbf{F} \boldsymbol{\sigma}^{\dagger *}-2 \mathbf{A}_{3}^{\prime(s)} \mathbf{F} \boldsymbol{\sigma}^{\dagger *}-\mathbf{A}_{3}^{\prime} \mathbf{F} 2 \mathbf{A}_{3}^{(s)} \\
& +\frac{2}{3} \mathbf{A}_{3}^{\prime} \mathbf{F} \mathbf{A}_{3}+\mathbf{A}_{2}^{\prime} \mathbf{F}_{\rho \pi}\left(\mathbf{A}_{\tilde{2}}-\mathbf{A}_{2}\right) \\
& +\mathbf{A}_{\tilde{2}}^{\prime} \mathbf{G}_{\rho} \boldsymbol{\Gamma}\left[\mathbf{G}\left(\mathbf{A}_{3}^{(u)}-\boldsymbol{\sigma}^{\dagger *}\right)-\mathbf{F} 2 \mathbf{A}_{3}^{(s)}\right]
\end{aligned}
$$

To obtain Eq. (93) we have made use of the following identities $(\checkmark N C A \checkmark)$ :

$$
\begin{gathered}
{\left[-\frac{2}{3}+\frac{1}{1-\mathbf{F T}}\right] \mathbf{F}=\mathbf{F}\left[\frac{1}{3}+\mathbf{T}_{L} \mathbf{F}\right],} \\
\frac{1}{1-\mathbf{T F}} \mathbf{T}=\frac{1}{1-\mathbf{K}_{2}(\mathbf{F}+\mathbf{G})} \mathbf{K}_{2}, \\
\frac{1}{1-\mathbf{T F}}(1+\mathbf{T G})=\frac{1}{1-\mathbf{K}_{2}(\mathbf{F}+\mathbf{G})},
\end{gathered}
$$

$$
\begin{gathered}
(1+\mathbf{G T}) \frac{1}{1-\mathbf{F T}}=\frac{1}{1-(\mathbf{F}+\mathbf{G}) \mathbf{K}_{2}} \\
{\left[\mathbf{G}+(1+\mathbf{G T}) \frac{1}{1-\mathbf{F T}} \mathbf{F}\right](1+\mathbf{T G})} \\
=\frac{1}{1-(\mathbf{F}+\mathbf{G}) \mathbf{K}_{2}}(\mathbf{F}+\mathbf{G})
\end{gathered}
$$

which follow from straightforward manipulations using the definitions Eqs. (45) and (70).

Equation (93) is equivalent to Eq. (83), where $\delta C_{\infty}^{\left[B_{2}\right],\{0\}}$ is understood as the $\mathcal{O}\left[\left(\mathbf{K}_{\mathrm{df}}\right)^{0}\right]$ contribution to $\delta C_{\infty}^{\left[B_{2}\right]}$. At this stage it remains only to show that $\delta C_{\infty}^{\left[B_{2}\right],\{0\}}$ only has exponentially suppressed volume dependence. This is done in Appendix B 3.

\section{G. $C_{\boldsymbol{L}}^{\left[\boldsymbol{B}_{2}\right]}$ to all orders in $\mathbf{K}_{\mathrm{df}}^{(\boldsymbol{u})}$ : Unsymmetrized}

In this subsection we collect the terms contributing to $C_{L}^{\left[B_{2}\right]}$, Eq. (49), that contain at least one factor of $\mathbf{K}_{\mathrm{df}}^{(u)}$. Throughout this subsection and the next, we use the superscript $\left[\mathbf{K}_{\mathrm{df}}\right]$ to denote the contribution to a quantity with one or more factors of the unsymmetrized divergencefree $\mathrm{K}$ matrix.

Beginning with $C_{L, 0 F}$, decomposed in Eq. (55), we use the results in Eqs. (59), (60), (65) and (67) and find that the part containing at least one factor of $\mathbf{K}_{\mathrm{df}}$ can be written as $(\sqrt{ } N C A \checkmark)$,

$$
\begin{aligned}
& C_{L, 0 F}^{\left[\mathbf{K}_{\mathrm{df}}\right]}=\left(2 \mathbf{A}_{3}^{\prime(s)} \mathbf{F}+\mathbf{A}_{\tilde{2}}^{\prime} \mathbf{G}_{\rho} \boldsymbol{\Gamma} \mathbf{G}\right)\left[\mathbf{K}_{L, 33}^{(u, u)}\left(\mathbf{F} 2 \mathbf{A}_{3}^{(s)}+\mathbf{G} \boldsymbol{\Gamma} \overline{\mathbf{G}}_{\rho} \mathbf{A}_{\tilde{2}}\right)+\mathbf{K}_{L, 3 \tilde{2}}^{(u)} \mathbf{F}_{\rho \pi} \mathbf{A}_{\tilde{2}}\right] \\
& +\mathbf{A}_{\tilde{2}}^{\prime} \mathbf{F}_{\rho \pi}\left[\mathbf{K}_{L, \tilde{2} 3}^{(u)}\left(\mathbf{F} 2 \mathbf{A}_{3}^{(s)}+\mathbf{G} \boldsymbol{\Gamma} \overline{\mathbf{G}}_{\rho} \mathbf{A}_{\tilde{2}}\right)+\mathbf{K}_{L, \tilde{2} \tilde{2}} \mathbf{F}_{\rho \pi} \mathbf{A}_{\tilde{2}}\right] \\
& =\left(\begin{array}{ll}
\mathbf{A}_{\tilde{2}}^{\prime} & 2 \mathbf{A}_{3}^{\prime(s)}
\end{array}\right)\left(\begin{array}{cc}
\mathbf{F}_{\rho \pi} & \mathbf{G}_{\rho} \boldsymbol{\Gamma} \mathbf{G} \\
0 & \mathbf{F}
\end{array}\right) \mathbf{K}_{L}^{(u)}\left(\begin{array}{cc}
\mathbf{F}_{\rho \pi} & 0 \\
\mathbf{G} \boldsymbol{\Gamma} \overline{\mathbf{G}}_{\rho} & \mathbf{F}
\end{array}\right)\left(\begin{array}{c}
\mathbf{A}_{\tilde{2}} \\
2 \mathbf{A}_{3}^{(s)}
\end{array}\right) \\
& =\left(\begin{array}{ll}
\mathbf{A}_{\tilde{2}}^{\prime} & 2 \mathbf{A}_{3}^{\prime(s)}
\end{array}\right)\left(\begin{array}{cc}
\mathbf{F}_{\rho \pi} & \mathbf{G}_{\rho} \boldsymbol{\Gamma} \mathbf{G} \\
0 & \mathbf{F}
\end{array}\right) \mathcal{E}_{L} \overline{\mathbf{V}} \mathbf{K}_{\mathrm{df}}^{(u)} \frac{1}{1-\mathbf{X K}_{\mathrm{df}}^{(u)}} \mathbf{V} \mathcal{E}_{R}\left(\begin{array}{cc}
\mathbf{F}_{\rho \pi} & 0 \\
\mathbf{G} \boldsymbol{\Gamma} \overline{\mathbf{G}}_{\rho} & \mathbf{F}
\end{array}\right)\left(\begin{array}{c}
\mathbf{A}_{\tilde{2}} \\
2 \mathbf{A}_{3}^{(s)}
\end{array}\right), \\
& =\left[\mathbf{A}_{\tilde{2}}^{\prime}\left(\begin{array}{ll}
1 & 0
\end{array}\right) \mathbf{X}+2 \mathbf{A}_{3}^{\prime(s)} \mathbf{F}(1+\mathbf{T G})\left(\boldsymbol{\Gamma} \overline{\mathbf{G}}_{\rho} 1\right)\right] \cdot \mathbf{K}_{\mathrm{df}}^{(u)} \frac{1}{1-\mathbf{X} \mathbf{K}_{\mathrm{df}}^{(u)}} \cdot\left[\mathbf{X}\left(\begin{array}{l}
1 \\
0
\end{array}\right) \mathbf{A}_{2}+\left(\begin{array}{c}
\mathbf{G}_{\rho} \boldsymbol{\Gamma} \\
1
\end{array}\right)(1+\mathbf{G} \mathbf{T}) \mathbf{F} 2 \mathbf{A}_{3}^{(s)}\right] .
\end{aligned}
$$

In Eq. (100) we have simply substituted Eqs. (59) and (60) into Eq. (55) and dropped terms that have no factors of $\mathbf{K}_{\mathrm{df}}^{(u)}$. To obtain Eq. (101) we then rearrange terms into a matrix form using the definition of $\mathbf{K}_{L}^{(u)}$, Eq. (64). Next we substitute the result Eq. (65) for $\mathbf{K}_{L}^{(u)}$, dropping terms with no factors of $\mathbf{K}_{\mathrm{df}}^{(u)}$, leading to Eq. (102). We then use the definition of $\mathbf{X}$, Eq. (67), to bring the result to the final form, Eq. (103).
We next turn to the terms in Eq. (49) that contain at least one factor of $\mathbf{F}_{33}^{(0)}$. These terms always include the end cap factors $\mathbf{A}_{L, 3}^{\prime(u)}$ and $\mathbf{A}_{L, 3}^{(u)}$ so that we first require the full decomposition of these. Beginning with $\mathbf{A}_{L, 3}^{\prime(u)}$, decomposed in Eq. (58), we insert the expressions for the different components of $\mathbf{K}_{L}^{(u)}$ [Eq. (65)] to find $(\sqrt{ } N C A \checkmark)$, 


$$
\begin{aligned}
& \mathbf{A}_{L, 3}^{\prime(u)}=\mathbf{A}_{3}^{\prime(u)}+\left(2 \mathbf{A}_{3}^{\prime(s)} \mathbf{F}+\mathbf{A}_{\tilde{2}}^{\prime} \mathbf{G}_{\rho} \boldsymbol{\Gamma} \mathbf{G}\right)\left(\mathbf{K}_{L, 33}^{(u, u)}+\mathbf{K}_{2}\right)+\mathbf{A}_{\tilde{2}}^{\prime}\left(\mathbf{F}_{\rho \pi} \mathbf{K}_{L, \tilde{2} 3}^{(u)}+\mathbf{G}_{\rho} \boldsymbol{\Gamma}\right), \\
& =\mathbf{A}_{3}^{\prime}-2 \mathbf{A}_{3}^{\prime(s)}+\left(2 \mathbf{A}_{3}^{\prime(s)} \mathbf{F}+\mathbf{A}_{\tilde{2}}^{\prime} \mathbf{G}_{\rho} \boldsymbol{\Gamma} \mathbf{G}\right)\left(\mathbf{K}_{L, 33}^{(0)}+\mathbf{K}_{2}\right)+\mathbf{A}_{\tilde{2}}^{\prime} \mathbf{G}_{\rho} \boldsymbol{\Gamma}+\left(2 \mathbf{A}_{3}^{\prime(s)} \mathbf{F}+\mathbf{A}_{2}^{\prime} \mathbf{G}_{\rho} \boldsymbol{\Gamma} \mathbf{G}\right)(1+\mathbf{T G})\left(\boldsymbol{\Gamma} \overline{\mathbf{G}}_{\rho} 1\right) \\
& \cdot \mathbf{K}_{\mathrm{df}}^{(u)} \frac{1}{1-\mathbf{X K}_{\mathrm{df}}^{(u)}} \cdot\left(\begin{array}{c}
\mathbf{G}_{\rho} \boldsymbol{\Gamma} \\
1
\end{array}\right)(1+\mathbf{G T})+\mathbf{A}_{\tilde{2}}^{\prime} \mathbf{F}_{\rho \pi}\left(\begin{array}{ll}
1 & 0
\end{array}\right) \cdot \mathbf{K}_{\mathrm{df}}^{(u)} \frac{1}{1-\mathbf{X} \mathbf{K}_{\mathrm{df}}^{(u)}} \cdot\left(\begin{array}{c}
\mathbf{G}_{\rho} \boldsymbol{\Gamma} \\
1
\end{array}\right)(1+\mathbf{G} \mathbf{T}), \\
& =\mathbf{A}_{3}^{\prime}-2 \mathbf{A}_{3}^{\prime(s)}(1-\mathbf{F T})+\mathbf{A}_{\tilde{2}}^{\prime}\left(\begin{array}{ll}
1 & 0
\end{array}\right) \cdot \frac{1}{1-\mathbf{X} \mathbf{K}_{\mathrm{df}}^{(u)}} \cdot\left(\begin{array}{c}
\mathbf{G}_{\rho} \boldsymbol{\Gamma} \\
1
\end{array}\right)(1+\mathbf{G T})
\end{aligned}
$$

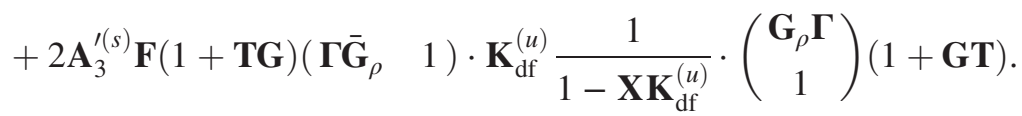

Here the first line is just a repeat of Eq. (58) and in the remaining lines we have substituted the expressions for $\mathbf{K}_{L, 33}^{(u, u)}$ and $\mathbf{K}_{L, \tilde{2} 3}^{(u)}$ and simplified.

The expression for the mirror-imaged end cap is then given by $(\boldsymbol{\checkmark} N C A \checkmark)$,

$$
\begin{aligned}
& \mathbf{A}_{L, 3}^{(u)}=\mathbf{A}_{3}-(1-\mathbf{T F}) 2 \mathbf{A}_{3}^{(s)}+(1+\mathbf{T G})\left(\boldsymbol{\Gamma} \overline{\mathbf{G}}_{\rho} 1\right) \cdot \frac{1}{1-\mathbf{K}_{\mathrm{df}}^{(u)} \mathbf{X}} \cdot\left(\begin{array}{l}
1 \\
0
\end{array}\right) \mathbf{A}_{\tilde{2}} \\
& +(1+\mathbf{T G})\left(\begin{array}{rr}
\mathbf{\Gamma} \overline{\mathbf{G}}_{\rho} & 1
\end{array}\right) \cdot \mathbf{K}_{\mathrm{df}}^{(u)} \frac{1}{1-\mathbf{X K}_{\mathrm{df}}^{(u)}} \cdot\left(\begin{array}{c}
\mathbf{G}_{\rho} \boldsymbol{\Gamma} \\
1
\end{array}\right)(1+\mathbf{G T}) \mathbf{F} 2 \mathbf{A}_{3}^{(s)} .
\end{aligned}
$$

In both cases we include the $\left(\mathbf{K}_{\mathrm{df}}^{(u)}\right)^{0}$ part, since the factors of $\mathbf{K}_{\mathrm{df}}^{(u)}$ can come from the sum appearing between the finitevolume end caps in the expression for $C_{L}^{\left[B_{2}\right]}$.

Finally, to derive an expression for the sum appearing between $\mathbf{A}_{L, 3}^{\prime(u)}$ and $\mathbf{A}_{L, 3}^{(u)}$ in Eq. (49), we make use of the following identity $(\sqrt{ } N C A \checkmark)$,

$$
\begin{aligned}
\mathbf{K}_{L, 33}^{(u, u)} & =\mathbf{K}_{L, 33}^{(0)}+\left(\begin{array}{ll}
0 & 1
\end{array}\right) \cdot \mathcal{E}_{L} \overline{\mathbf{V}} \mathbf{K}_{\mathrm{df}}^{(u)} \frac{1}{1-\mathbf{X} \mathbf{K}_{\mathrm{df}}^{(u)}} \mathbf{V} \mathcal{E}_{R} \cdot\left(\begin{array}{c}
0 \\
1
\end{array}\right), \\
& =\mathbf{K}_{L, 33}^{(0)}+\left(\begin{array}{ll}
1+\mathbf{T} \mathbf{G})\left(\mathbf{\Gamma} \overline{\mathbf{G}}_{\rho}\right. & 1
\end{array}\right) \cdot \mathbf{K}_{\mathrm{df}}^{(u)} \frac{1}{1-\mathbf{X} \mathbf{K}_{\mathrm{df}}^{(u)}} \cdot\left(\begin{array}{c}
\mathbf{G}_{\rho} \boldsymbol{\Gamma} \\
1
\end{array}\right)(1+\mathbf{G T}), \\
& \equiv \mathbf{K}_{L, 33}^{(0)}+\mathbf{K}_{L, 33}^{(u, u),\left[\mathbf{K}_{\mathrm{df}}\right]},
\end{aligned}
$$

where $\mathbf{K}_{L, 33}^{(u, u),\left[\mathbf{K}_{\mathrm{df}}\right]}$ is defined by comparing Eqs. (109) and (110).

Combining this with the expression for $\mathbf{Z}$, defined in Eq. (84), we find $(\checkmark N C A \checkmark)$

$$
\begin{aligned}
\mathbf{F}_{33}^{(0)} \frac{1}{1-\mathbf{K}_{L, 33}^{(u, u)} \mathbf{F}_{33}^{(0)}} & =\mathbf{Z} \frac{1}{1-\mathbf{K}_{L, 33}^{(u, u),\left[\mathbf{K}_{\mathrm{df}}\right]} \mathbf{Z}}, \\
& =\mathbf{Z}+\mathbf{Z} \mathbf{K}_{L, 33}^{(u, u),\left[\mathbf{K}_{\mathrm{df}}\right]} \sum_{n=0}^{\infty}\left(\mathbf{Z} \mathbf{K}_{L, 33}^{(u, u),\left[\mathbf{K}_{\mathrm{df}}\right]}\right)^{n} \mathbf{Z} \\
& =\mathbf{Z}+\mathbf{Z}(1+\mathbf{T G})\left(\mathbf{\Gamma} \overline{\mathbf{G}}_{\rho} 1\right) \cdot \mathbf{K}_{\mathrm{df}}^{(u)} \frac{1}{1-\mathbf{X} \mathbf{K}_{\mathrm{df}}^{(u)}} \sum_{n=0}^{\infty}\left(\mathbf{Y} \mathbf{K}_{\mathrm{df}}^{(u)} \frac{1}{1-\mathbf{X K}_{\mathrm{df}}^{(u)}}\right)^{n} \cdot\left(\begin{array}{c}
\mathbf{G}_{\rho} \boldsymbol{\Gamma} \\
1
\end{array}\right)(1+\mathbf{G T}) \mathbf{Z}, \\
& =\mathbf{Z}+\mathbf{Z}(1+\mathbf{T G})\left(\mathbf{\Gamma} \overline{\mathbf{G}}_{\rho} \quad 1\right) \cdot \mathbf{K}_{\mathrm{df}}^{(u)} \frac{1}{1-(\mathbf{X}+\mathbf{Y}) \mathbf{K}_{\mathrm{df}}^{(u)}} \cdot\left(\begin{array}{c}
\mathbf{G}_{\rho} \boldsymbol{\Gamma} \\
1
\end{array}\right)(1+\mathbf{G} \mathbf{T}) \mathbf{Z},
\end{aligned}
$$

where the new matrix $\mathbf{Y}$ is $(\boldsymbol{V N C A} \checkmark)$

$$
\mathbf{Y} \equiv\left(\begin{array}{c}
\mathbf{G}_{\rho} \boldsymbol{\Gamma} \\
1
\end{array}\right) \cdot(1+\mathbf{G} \mathbf{T}) \mathbf{Z}(1+\mathbf{T G}) \cdot\left(\begin{array}{ll}
\boldsymbol{\Gamma} \overline{\mathbf{G}}_{\rho} & 1
\end{array}\right),
$$




$$
=\left(\begin{array}{c}
\mathbf{G}_{\rho} \boldsymbol{\Gamma} \\
1
\end{array}\right) \cdot\left[-\mathbf{G}_{\mathbf{K}}+\frac{1}{1-(\mathbf{F}+\mathbf{G}) \mathbf{K}_{2}}(\mathbf{F}+\mathbf{G})\right] \cdot\left(\begin{array}{ll}
\boldsymbol{\Gamma} \overline{\mathbf{G}}_{\rho} & 1
\end{array}\right) .
$$

From this, together with the expression for $\mathbf{X}$ [Eq. (67)] and $\mathbf{F}_{\tilde{2} \tilde{2}}$ [Eq. (29)], we find that the combined matrix appearing between factors of $\mathbf{K}_{\mathrm{df}}^{(u)}$ is $(\boldsymbol{} N C A \checkmark)$

$$
\mathbf{X}+\mathbf{Y}=\left(\begin{array}{cc}
\mathbf{F}_{\tilde{2} \tilde{2}} & \mathbf{G}_{\rho} \boldsymbol{\Gamma} \frac{1}{1-(\mathbf{F}+\mathbf{G}) \mathbf{K}_{2}}(\mathbf{F}+\mathbf{G}) \\
(\mathbf{F}+\mathbf{G}) \frac{1}{1-\mathbf{K}_{2}(\mathbf{F}+\mathbf{G})} \boldsymbol{\Gamma} \overline{\mathbf{G}}_{\rho} & \frac{1}{1-(\mathbf{F}+\mathbf{G}) \mathbf{K}_{2}}(\mathbf{F}+\mathbf{G})
\end{array}\right) .
$$

We observe that the off-diagonal elements are close to $\mathbf{F}_{\tilde{2} 3}$ and $\mathbf{F}_{32}$, differing only by the presence of $\mathbf{F}+\mathbf{G}$ rather than $\mathbf{F}$ on the ends. Similarly, the 33 element is close to $\mathbf{F}_{33}$. These differences are removed when we change from the unsymmetrized $\mathbf{K}_{\mathrm{df}}^{(u)}$ to the symmetrized version.

With these preliminaries, we begin the determination of $C_{L}^{\left[B_{2}\right],\left[\mathbf{K}_{\mathrm{df}}\right]}$ by collecting the terms involving factors of $\mathbf{A}_{\tilde{2}}^{\prime}$ and $\mathbf{A}_{\tilde{2}}$ on the ends. All terms appearing in Eq. (103) as well as the appropriate combinations of Eqs. (106), (107) and (114) that contain these end caps have the form

$$
C_{L}^{\left[B_{2}\right],\left[\mathbf{K}_{\mathrm{df}}\right]} \supset \mathbf{A}_{\tilde{2}}^{\prime}\left(\begin{array}{ll}
1 & 0
\end{array}\right) \cdot \mathbf{W} \cdot\left(\begin{array}{l}
1 \\
0
\end{array}\right) \mathbf{A}_{\tilde{2}}
$$

and our task is to determine the matrix $\mathbf{W}$. Collecting terms, we find $^{14}(\checkmark N C A \checkmark)$

$$
\begin{aligned}
\mathbf{W}= & \mathbf{X} \mathbf{K}_{\mathrm{df}}^{(u)} \frac{1}{1-\mathbf{X} \mathbf{K}_{\mathrm{df}}^{(u)}} \mathbf{X}+\frac{1}{1-\mathbf{X} \mathbf{K}_{\mathrm{df}}^{(u)}} \\
& \times\left(\mathbf{Y}+\mathbf{Y} \mathbf{K}_{\mathrm{df}}^{(u)} \frac{1}{1-(\mathbf{X}+\mathbf{Y}) \mathbf{K}_{\mathrm{df}}^{(u)}} \mathbf{Y}\right) \frac{1}{1-\mathbf{K}_{\mathrm{df}}^{(u)} \mathbf{X}}-\mathbf{Y} \\
= & (\mathbf{X}+\mathbf{Y}) \mathbf{K}_{\mathrm{df}}^{(u)} \frac{1}{1-(\mathbf{X}+\mathbf{Y}) \mathbf{K}_{\mathrm{df}}^{(u)}}(\mathbf{X}+\mathbf{Y})
\end{aligned}
$$

Next we consider the cases with either $\mathbf{A}_{3}^{\prime(s)}$ on the lefthand side, or $\mathbf{A}_{3}^{(s)}$ on the right, or both. After some algebra, we find that all such terms vanish identically.

The remaining, nonvanishing terms are those involving the end caps $\mathbf{A}_{3}^{\prime}$ and $\mathbf{A}_{3}$. We find

$$
\begin{aligned}
C_{L}^{\left[B_{2}\right],\left[\mathbf{K}_{\mathrm{df}}\right]} \supset \mathbf{A}_{3}^{\prime} \mathbf{Z}(1+\mathbf{T G})\left(\mathbf{\Gamma} \overline{\mathbf{G}}_{\rho} 1\right) \cdot \mathbf{K}_{\mathrm{df}}^{(u)} \frac{1}{1-(\mathbf{X}+\mathbf{Y}) \mathbf{K}_{\mathrm{df}}^{(u)}}(\mathbf{X}+\mathbf{Y}) \cdot\left(\begin{array}{c}
1 \\
0
\end{array}\right) \mathbf{A}_{\tilde{2}}+\mathbf{A}_{\tilde{2}}^{\prime}(1 \quad 0) \cdot(\mathbf{X}+\mathbf{Y}) \mathbf{K}_{\mathrm{df}}^{(u)} \frac{1}{1-(\mathbf{X}+\mathbf{Y}) \mathbf{K}_{\mathrm{df}}^{(u)}} \\
\cdot\left(\begin{array}{c}
\mathbf{G}_{\rho} \boldsymbol{\Gamma} \\
1
\end{array}\right)(1+\mathbf{G T}) \mathbf{Z} \mathbf{A}_{3}+\mathbf{A}_{3}^{\prime} \mathbf{Z}(1+\mathbf{T G})\left(\boldsymbol{\Gamma} \overline{\mathbf{G}}_{\rho} 1\right) \cdot \mathbf{K}_{\mathrm{df}}^{(u)} \frac{1}{1-(\mathbf{X}+\mathbf{Y}) \mathbf{K}_{\mathrm{df}}^{(u)}} \cdot\left(\begin{array}{c}
\mathbf{G}_{\rho} \boldsymbol{\Gamma} \\
1
\end{array}\right)(1+\mathbf{G T}) \mathbf{Z} \mathbf{A}_{3} \cdot
\end{aligned}
$$

Finally, we can combine the results in Eqs. (118) and (121) into a compact matrix form,

$$
C_{L}^{\left[B_{2}\right],\left[\mathbf{K}_{\mathrm{df}}\right]}=\left(\begin{array}{ll}
\mathbf{A}_{\tilde{2}}^{\prime} & \mathbf{A}_{3}^{\prime}
\end{array}\right) \cdot \mathbf{F}_{\mathbf{L}} \mathbf{K}_{\mathrm{df}}^{(u)} \frac{1}{1-(\mathbf{X}+\mathbf{Y}) \mathbf{K}_{\mathrm{df}}^{(u)}} \mathbf{F}_{\mathbf{R}} \cdot\left(\begin{array}{c}
\mathbf{A}_{\tilde{2}} \\
\mathbf{A}_{3}
\end{array}\right),
$$

where we have introduced

$$
\begin{aligned}
& \mathbf{F}_{\mathbf{L}} \equiv\left(\begin{array}{cc}
\mathbf{F}_{\tilde{2} \tilde{2}} & \mathbf{X}_{\tilde{2} 3}+\mathbf{Y}_{\tilde{2} 3} \\
\mathbf{F}_{3 \tilde{2}} & \mathbf{F}_{1-\mathbf{K}_{2}(\mathbf{F}+\mathbf{G})}
\end{array}\right) \text { and } \\
& \mathbf{F}_{\mathbf{R}} \equiv\left(\begin{array}{cc}
\mathbf{F}_{\tilde{2} \tilde{2}} & \mathbf{F}_{\tilde{2} 3} \\
\mathbf{X}_{3 \tilde{2}}+\mathbf{Y}_{3 \tilde{2}} & \frac{1}{1-(\mathbf{F}+\mathbf{G}) \mathbf{K}_{2}} \mathbf{F}
\end{array}\right)
\end{aligned}
$$

\footnotetext{
${ }^{14} \mathrm{On}$ the right-hand side of the first equality, the final $-\mathbf{Y}$ term is needed to remove the $\left(\mathbf{K}_{\mathrm{df}}^{(u)}\right)^{0}$ contribution to the previous term.
}

To obtain this form, we have used the identities (97) and (98), as well as the definitions of $\mathbf{F}_{\tilde{2} 3}$ and $\mathbf{F}_{32}$, given in Eqs. (47) and (48), respectively.

\section{H. Symmetrization of $\mathbf{K}_{\mathrm{df}}$ in $C_{L}^{\left[\boldsymbol{B}_{2}\right]}$}

A pleasing feature of the result of the previous section, Eq. (122), is that it contains only symmetrized end caps, despite the presence of unsymmetrized end caps at earlier stages. It does, however, contain the unsymmetrized quantity $\mathbf{K}_{\mathrm{df}}^{(u)}$, and in this section we manipulate the result so that all infinite-volume quantities have the desired exchange symmetry. Here we build upon the work of 


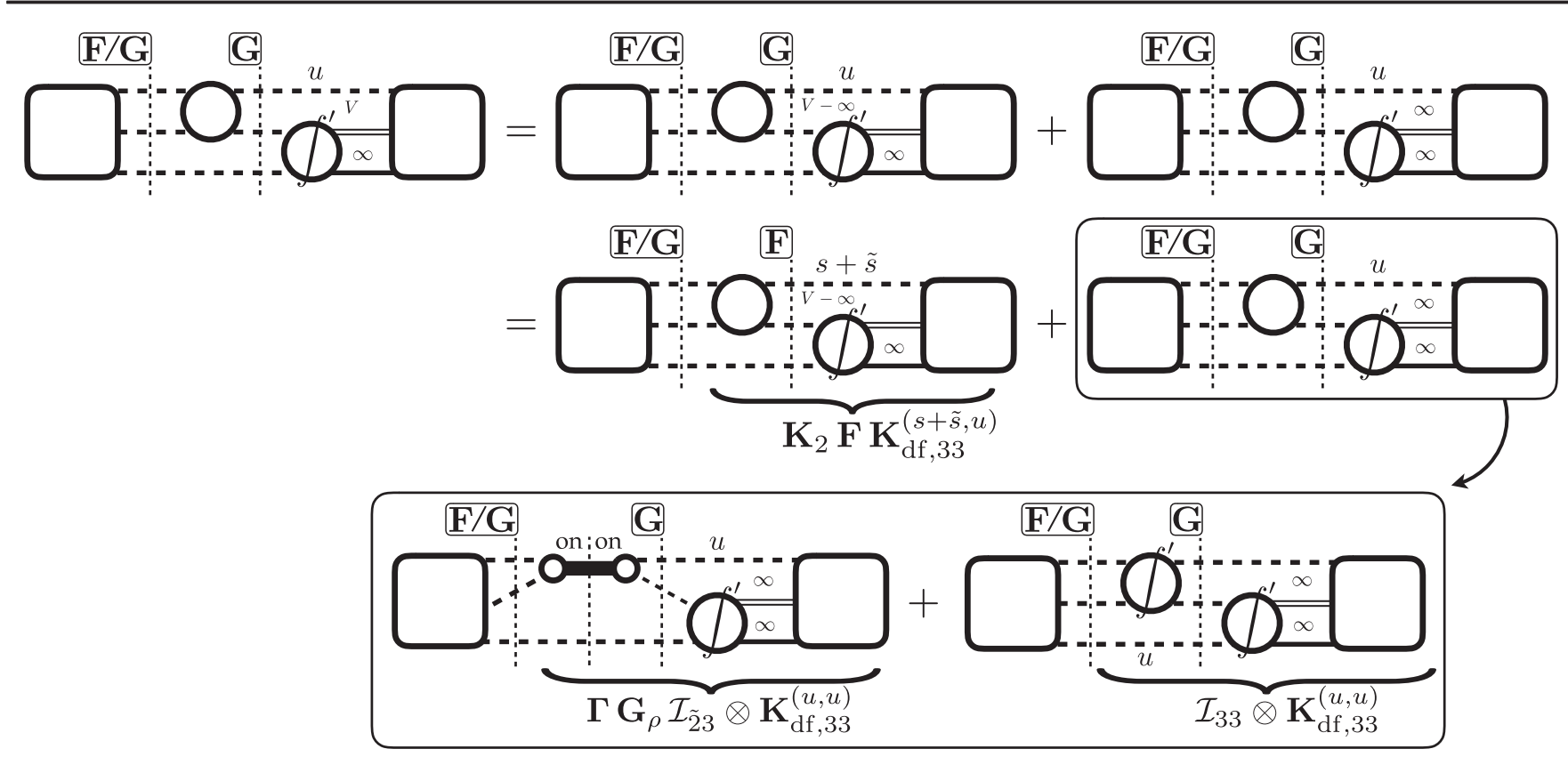

FIG. 4. Derivation of Eq. (125), using the notation of Figs. 1 and 7. On the left-hand side of the equality, the quantity to the right of the $G$ cut is $\mathbf{K}_{\mathrm{df}, 33}^{(u, u)}$, with the $u$ above the upper-right dashed line indicating that this is an unsymmetrized quantity. The left-hand cut in all diagrams must be present, but can be either $\mathbf{F}$ or $\mathbf{G}$. The box at the left-hand end of each diagram represents whatever lies to the left of the $F / G$ cut, which depends on the context, but for which the details are irrelevant. The first two equalities show how $\mathbf{G}$ is converted to $\mathbf{F}$ by adding and subtracting an integral. This method is used extensively in Ref. [5] and is explained in Eqs. (163)-(165) of that work and the accompanying text. It results in the $u$ superscript on $\mathbf{K}_{\mathrm{df}, 33}$ changing to $s+\tilde{s}$ in the $\mathbf{F}$ term. In the second step (indicated by the arrow connecting the two boxed diagrams), $\mathcal{K}_{2}$ is replaced by the pole term, with on-shell projection onto the K-matrix pole, and the smooth part. Since there is now an integral to the right of the $\mathbf{G}$, rather than a sum, the infinite-volume quantity $\mathbf{K}_{\mathrm{df}, 33}^{(u, u)}$ is extended to the left by the addition of either a $\Gamma$ or $\mathcal{K}_{2}^{\prime}$, implicitly defining the integral operators $\mathcal{I}_{23}$ and $\mathcal{I}_{33}$, respectively.

Ref. [5], but again need additional techniques to deal with the poles in $\mathcal{K}_{2}$. We also have found ways to shorten the argumentation given in Ref. [5]. Nevertheless, this section is the most algebraically involved in this work.

A key observation for doing the symmetrization is that, if Eq. (122) is expanded in powers of $\mathbf{K}_{\mathrm{df}}^{(u)}$, then in all terms with more than one factor of this unsymmetrized threeparticle quantity, it always lies next to a factor of $\mathbf{F}+\mathbf{G}$, due to the structure of $\mathbf{X}+\mathbf{Y}$, Eq. (117). This allows us to use a class of symmetrization results exemplified by

$$
\begin{aligned}
\mathbf{K}_{2}(\mathbf{F}+\mathbf{G}) \mathbf{K}_{\mathrm{df}, 33}^{(u, u)}= & \mathbf{K}_{2} \mathbf{F} \mathcal{S}\left[\mathbf{K}_{\mathrm{df}, 33}^{(u, u)}\right]+\boldsymbol{\Gamma} \overline{\mathbf{G}}_{\rho} \mathcal{I}_{\tilde{2} 3} \otimes \mathbf{K}_{\mathrm{df}, 33}^{(u, u)} \\
& +\mathcal{I}_{33} \otimes \mathbf{K}_{\mathrm{df}, 33}^{(u, u)}
\end{aligned}
$$

where $\mathcal{S}$ is the symmetrization operator that converts a $(u)$ quantity into the symmetric $(u+s+\tilde{s})$ version, ${ }^{15}$ while $\mathcal{I}_{\tilde{2} 3}$ and $\mathcal{I}_{33}$ are integral operators, to be explained below. The result (124) holds with $\mathbf{K}_{\mathrm{df}, 33}^{(u, u)}$ replaced by any threeparticle quantity with the $(u)$ superscript, e.g., $\mathbf{K}_{\mathrm{df}, 3 \tilde{2}}^{(u)}$. It also assumes that there is at least one factor of $\mathbf{F}$ or $\mathbf{G}$ on the left,

\footnotetext{
${ }^{15}$ Here $\mathcal{S}$ acts to the right, but, in the following, it also acts to the left. Which is the case is clear from the context.
}

as is true in general because $(\mathbf{X}+\mathbf{Y}), \mathbf{F}_{\mathbf{L}}$ and $\mathbf{F}_{\mathbf{R}}$ contain the geometric series $1 /\left(1-(\mathbf{F}+\mathbf{G}) \mathbf{K}_{2}\right)$.

To demonstrate Eq. (124) we derive the equivalent result

$$
\begin{aligned}
\mathbf{K}_{2} \mathbf{G} \mathbf{K}_{\mathrm{df}, 33}^{(u, u)}= & \mathbf{K}_{2} \mathbf{F} \mathbf{K}_{\mathrm{df}, 33}^{(s+\tilde{s}, u)}+\boldsymbol{\Gamma} \overline{\mathbf{G}}_{\rho} \mathcal{I}_{\tilde{2} 3} \otimes \mathbf{K}_{\mathrm{df}, 33}^{(u, u)} \\
& +\mathcal{I}_{33} \otimes \mathbf{K}_{\mathrm{df}, 33}^{(u, u)},
\end{aligned}
$$

in Fig. 4. As seen from the figure, the integral operator $\mathcal{I}_{\tilde{2} 3}$, attaches a factor of $\boldsymbol{\Gamma}$ to $\mathbf{K}_{\mathrm{df}, 33}^{(u, u)}$, leading to an infinitevolume two-particle quantity, while $\mathcal{I}_{33}$ attaches $i \tilde{\mathcal{K}}_{2}^{\prime}$ to $\mathbf{K}_{\mathrm{df}, 33}^{(u, u)}$, creating another infinite-volume three-particle quantity with the $(u)$ superscript.

The reflected equation is derived similarly and is

$$
\begin{aligned}
\mathbf{K}_{\mathrm{df}, 33}^{(u, u)}(\mathbf{F}+\mathbf{G}) \mathbf{K}_{2}= & {\left[\mathbf{K}_{\mathrm{df}, 33}^{(u, u)}\right] \mathcal{S} \mathbf{F} \mathbf{K}_{2}+\mathbf{K}_{\mathrm{df}, 33}^{(u, u)} } \\
& \otimes \mathcal{I}_{32} \mathbf{G}_{\rho} \boldsymbol{\Gamma}+\mathbf{K}_{\mathrm{df}, 33}^{(u, u)} \otimes \mathcal{I}_{33}^{\dagger},
\end{aligned}
$$

where $\mathcal{I}_{3 \tilde{2}}$ and $\mathcal{I}_{33}^{\dagger}$ are integral operators acting to the left on three-particle unsymmetrized quantities. The direction of action of the integral operators is indicated by the position of the $\otimes$ symbol. 
We can iterate Eq. (124), assuming implicitly that it acts on an unsymmetrized three-particle quantity on the right, and that there are additional implicit factors of $\mathbf{F}$ or $\mathbf{G}$ on the left. We find

$$
\begin{aligned}
\frac{1}{1-\mathbf{K}_{2}(\mathbf{F}+\mathbf{G})}= & \sum_{n=0}^{\infty}\left\{\mathbf{K}_{2}(\mathbf{F}+\mathbf{G})\right\}^{n} \\
= & \frac{1}{1-\mathbf{K}_{2}(\mathbf{F}+\mathbf{G})}\left\{\mathbf{K}_{2} \mathbf{F} \mathcal{S}+\mathbf{\Gamma} \overline{\mathbf{G}}_{\rho} \mathcal{I}_{\tilde{2} 3} \otimes\right\} \\
& \times \frac{1}{1-\mathcal{I}_{33} \otimes}+\frac{1}{1-\mathcal{I}_{33} \otimes} .
\end{aligned}
$$

The first term in curly braces on the right-hand side leads to symmetrized quantities (since it contains the operator $\mathcal{S}$ ), while the second, $\boldsymbol{\Gamma}$-dependent term does not require symmetrization. The final term on the right-hand side of this result is an unsymmetrized residue that is dealt with subsequently.

We now apply this result to the quantity of interest, $C_{L}^{\left[B_{2}\right],\left[\mathbf{K}_{\mathrm{df}}\right]}$ in Eq. (122). We begin by considering the contribution in which cuts appear between the end caps $\mathbf{A}_{3}^{\prime}$ and $\mathbf{A}_{3}$ and the outermost $\mathbf{K}_{\mathrm{df}}^{(u)}$ insertions. Here the analysis is simplified by having a symmetrized quantity on one side. Focusing first on the right-side end cap, we find

$$
\begin{aligned}
& \quad \mathbf{A}_{3}^{\prime}\left[\mathbf{F}_{\mathbf{L}}\right]_{33} \mathbf{K}_{\mathrm{df}, 3 \tilde{2}}^{(u)}=\mathbf{A}_{3}^{\prime} \mathbf{F} \frac{1}{1-\mathbf{K}_{2}(\mathbf{F}+\mathbf{G})} \mathbf{K}_{\mathrm{df}, 3 \tilde{2}}^{(u)}, \\
& =\mathbf{A}_{3}^{\prime} \mathbf{F} \frac{1}{1-\mathcal{I}_{33} \otimes} \mathbf{K}_{\mathrm{df}, 3 \tilde{2}}^{(u)}+\mathbf{A}_{3}^{\prime} \mathbf{F} \frac{1}{1-\mathbf{K}_{2}(\mathbf{F}+\mathbf{G})} \\
& \times\left\{\mathbf{K}_{2} \mathbf{F} \mathcal{S}+\mathbf{\Gamma} \overline{\mathbf{G}}_{\rho} \mathcal{I}_{\tilde{2} 3} \otimes\right\} \frac{1}{1-\mathcal{I}_{33} \otimes} \mathbf{K}_{\mathrm{df}, 3 \tilde{2}}^{(u)}, \\
& =\frac{1}{3} \mathbf{A}_{3}^{\prime} \mathbf{F} \mathbf{K}_{\mathrm{df}, 3 \tilde{2}}+\mathbf{A}_{3}^{\prime} \mathbf{F} \frac{1}{1-\mathbf{K}_{2}(\mathbf{F}+\mathbf{G})} \mathbf{K}_{2} \mathbf{F} \mathbf{K}_{\mathrm{df}, 3 \tilde{2}} \\
& +\mathbf{A}_{3}^{\prime} \mathbf{F}_{3 \tilde{2}} \delta \mathbf{K}_{\mathrm{df}, \tilde{2} \tilde{2}}+\delta_{33} \mathbf{A}_{\tilde{2}}^{\prime}, \\
& =\mathbf{A}_{3}^{\prime} \mathbf{F}_{33} \mathbf{K}_{\mathrm{df}, 3 \tilde{2}}+\mathbf{A}_{3}^{\prime} \mathbf{F}_{3 \tilde{2}} \delta \mathbf{K}_{\mathrm{df}, \tilde{2} \tilde{2}}+\delta_{33} \mathbf{A}_{\tilde{2}}^{\prime} .
\end{aligned}
$$

The first line recalls the definition of $\mathbf{F}_{\mathbf{L}}$, while the second substitutes Eq. (127). To obtain the third line we use the definition of $\mathbf{F}_{32}$ as well as the following new definitions:

$$
\begin{gathered}
\mathbf{K}_{\mathrm{df}, 3 \tilde{2}} \equiv \mathcal{S} \frac{1}{1-\mathcal{I}_{33} \otimes} \mathbf{K}_{\mathrm{df}, 3 \tilde{2}}^{(u)}, \\
\delta \mathbf{K}_{\mathrm{df}, \tilde{2} \tilde{2}} \equiv \mathcal{I}_{\tilde{2} 3} \otimes \frac{1}{1-\mathcal{I}_{33} \otimes} \mathbf{K}_{\mathrm{df}, 3 \tilde{2}}^{(u)}, \\
\delta_{33} \mathbf{A}_{\tilde{2}}^{\prime} \equiv \mathbf{A}_{3}^{\prime} \frac{i \rho}{3 \omega}\left(\frac{1}{1-\mathcal{I}_{33} \otimes} \mathbf{K}_{\mathrm{df}, 3 \tilde{2}}^{(u)}\right)^{(u-s)} .
\end{gathered}
$$

In addition we use the result from Ref. [5] that a factor of $\mathbf{F}$ sandwiched between a symmetric object (here $\mathbf{A}_{3}^{\prime}$ ) and a $(u-s)$ object can be replaced by $i \rho /(2 \omega)$, so that the resulting matrix sum can be replaced by an integral. The final line follows immediately using the definition of $\mathbf{F}_{33}$. We see that the symmetrization has produced the desired factors of $\mathbf{F}_{3 \tilde{2}}$ and $\mathbf{F}_{33}$, as well as an additional contribution to $\mathbf{K}_{\mathrm{df}, \tilde{2} \tilde{2}}$ and to the end cap $\mathbf{A}_{\tilde{2}}^{\prime}$. An almost identical set of results holds with $\mathbf{K}_{\mathrm{df}, 3 \tilde{2}}^{(u)}$ replaced with $\mathbf{K}_{\mathrm{df}, 33}^{(u, u)}$, except that the final index is changed from $\tilde{2}$ to 3 , and an additional $(u)$ superscript is added.

We next consider terms where the end cap is $\mathbf{A}_{\tilde{2}}^{\prime}$ or its reflection. In this case we need a slightly different symmetrization result,

$$
\begin{aligned}
\mathbf{A}_{\tilde{2}}^{\prime} \mathbf{G}_{\rho} \boldsymbol{\Gamma}(\mathbf{F}+\mathbf{G}) \mathbf{K}_{\mathrm{df}, 3 \tilde{2}}^{(u)} & \\
= & \mathbf{A}_{\tilde{2}}^{\prime} \mathbf{G}_{\rho} \boldsymbol{\Gamma} \mathbf{F} \mathcal{S}\left[\mathbf{K}_{\mathrm{df}, 3 \tilde{2}}^{(u)}\right]+\mathbf{A}_{\tilde{2}}^{\prime} \mathbf{F}_{\rho \pi} \mathcal{I}_{\tilde{2} 3} \otimes \mathbf{K}_{\mathrm{df}, 3 \tilde{2}}^{(u)} \\
& +\mathbf{A}_{\tilde{2}}^{\prime} \otimes \rho_{\tilde{2} 3} \otimes \mathbf{K}_{\mathrm{df}, 3 \tilde{2}}^{(u)} .
\end{aligned}
$$

This follows from

$$
\begin{aligned}
\mathbf{A}_{\tilde{2}}^{\prime} \mathbf{G}_{\rho} \boldsymbol{\Gamma} \mathbf{G K}_{\mathrm{df}, 3 \tilde{2}}^{(u)}= & \mathbf{A}_{\tilde{2}}^{\prime} \mathbf{G}_{\rho} \boldsymbol{\Gamma} \mathbf{F} \mathbf{K}_{\mathrm{df}, 3 \tilde{2}}^{(s+\tilde{s})}+\mathbf{A}_{\tilde{2}}^{\prime} \mathbf{F}_{\rho \pi} \mathcal{I}_{\tilde{2} 3} \otimes \mathbf{K}_{\mathrm{df}, 3 \tilde{2}}^{(u)} \\
& +\mathbf{A}_{\tilde{2}}^{\prime} \otimes \rho_{\tilde{2} 3} \otimes \mathbf{K}_{\mathrm{df}, 3 \tilde{2}}^{(u)},
\end{aligned}
$$

the derivation of which is described in Fig. 5. Here $\rho_{2} 3$ is a second type of integral operator that acts both to the left and right, and is defined in the figure. It joins $\mathbf{A}_{\tilde{2}}^{\prime}$ with $\mathbf{K}_{\mathrm{df}, 3 \tilde{2}}^{(u)}$ into an expanded end cap. We stress that the results in Eqs. (135) and (136) hold when $\mathbf{K}_{\mathrm{df}, 3 \tilde{2}}^{(u)}$ is replaced by any unsymmetrized three-particle quantity.

Using the definition of $\mathbf{F}_{\mathbf{L}}$ [Eq. (123)] and Eqs. (127) and (135), we find

$$
\mathbf{A}_{\tilde{2}}^{\prime}\left[\mathbf{F}_{\mathbf{L}}\right]_{\tilde{2} 3} \mathbf{K}_{\mathrm{df}, 3 \tilde{2}}^{(u)}=\mathbf{A}_{\tilde{2}}^{\prime} \mathbf{G}_{\rho} \boldsymbol{\Gamma} \frac{1}{1-(\mathbf{F}+\mathbf{G}) \mathbf{K}_{2}}(\mathbf{F}+\mathbf{G}) \mathbf{K}_{\mathrm{df}, 3 \tilde{2}}^{(u)},
$$

$$
\begin{aligned}
& =\mathbf{A}_{\tilde{2}}^{\prime} \mathbf{G}_{\rho} \boldsymbol{\Gamma}(\mathbf{F}+\mathbf{G})\left\{\frac{1}{1-\mathbf{K}_{2}(\mathbf{F}+\mathbf{G})}\left(\mathbf{K}_{2} \mathbf{F} \mathcal{S}+\mathbf{\Gamma} \overline{\mathbf{G}}_{\rho} \mathcal{I}_{\tilde{2} 3} \otimes\right)+1\right\} \\
& \times \frac{1}{1-\mathcal{I}_{33} \otimes} \mathbf{K}_{\mathrm{df}, 3 \tilde{2}}^{(u)}, \\
& \quad=\mathbf{A}_{\tilde{2}}^{\prime} \mathbf{F}_{\tilde{2} 3} \mathbf{K}_{\mathrm{df}, 3 \tilde{2}}+\mathbf{A}_{\tilde{2}}^{\prime} \mathbf{F}_{\tilde{2} \tilde{2}} \delta \mathbf{K}_{\mathrm{df}, \tilde{2} \tilde{2}}+\delta_{\tilde{2} 3} \mathbf{A}_{\tilde{2}}^{\prime},
\end{aligned}
$$

where

$$
\delta_{\tilde{2} 3} \mathbf{A}_{\tilde{2}}^{\prime} \equiv \mathbf{A}_{\tilde{2}}^{\prime} \otimes \rho_{\tilde{2} 3} \otimes \frac{1}{1-\mathcal{I}_{33} \otimes} \mathbf{K}_{\mathrm{df}, 3 \tilde{2}}^{(u)} .
$$




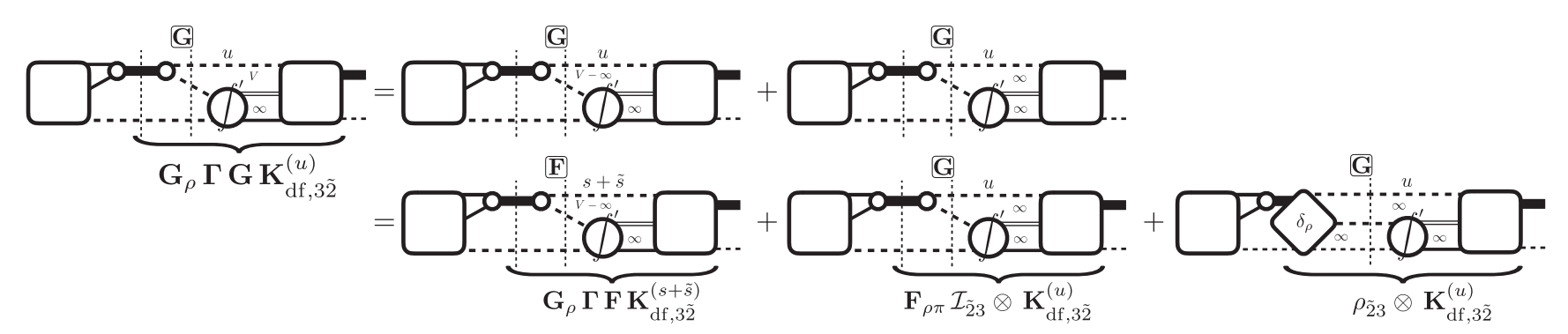

FIG. 5. Derivation of Eq. (136), using the notation of Figs. 1 and 7. The left-hand boxes represent $\mathbf{A}_{\tilde{2}}^{\prime}$, aside from the loop that is exposed explicitly to the left of the leftmost cut. The steps are similar to those in Fig. 4: replacing the sum adjacent to the $\mathbf{G}$ with a sumintegral difference and an integral, the former giving rise to an $\mathbf{F}$. The difference from Fig. 4 concerns the integral, in which the factor of $\mathbf{G}_{\rho}$ can be converted into an $\mathbf{F}_{\rho \pi}$ cut by projecting the entire quantity to the right onto the K-matrix pole onto the $\tilde{2}$ mass shell, leading to the $\mathcal{I}_{23}$ term. The residue (the $\delta_{\rho}$ term) cancels the K-matrix pole, allowing the sum over the momentum $k$ to be replaced by an integral, so that the implicit $\mathbf{A}_{\tilde{2}}^{\prime}$ and the $\mathbf{K}_{\mathrm{df}, 3 \tilde{2}}^{(u)}$ are connected by an infinite-volume integral operator denoted $\rho_{\tilde{2} 3}$.

As above, an almost identical equation holds with $\mathbf{K}_{\mathrm{df}, 3 \tilde{2}}^{(u)}$ replaced by $\mathbf{K}_{\mathrm{df}, 33}^{(u, u)}$.

Combining Eqs. (131) and (139) and their analogs with the right-hand index changed to 3 , we find

$$
\mathbf{A}^{\prime} \mathbf{F}_{\mathbf{L}} \mathbf{K}_{\mathrm{df}}^{(u)}=\mathbf{A}^{\prime} \mathcal{F} \mathcal{S}_{\mathbf{L}}\left[\mathbf{K}_{\mathrm{df}}^{(u)}\right]+\left(\begin{array}{ll}
\delta \mathbf{A}_{\tilde{2}}^{\prime} & \delta \mathbf{A}_{3}^{\prime(u)}
\end{array}\right),
$$

where $\mathcal{F}$ is defined in Eq. (44),

$$
\mathbf{A}^{\prime} \equiv\left(\begin{array}{ll}
\mathbf{A}_{\tilde{2}}^{\prime} & \mathbf{A}_{3}^{\prime}
\end{array}\right), \quad \mathcal{S}_{\mathbf{L}} \equiv\left(\begin{array}{cc}
1 & \mathcal{I}_{\tilde{2} 3} \otimes \frac{1}{1-\mathcal{I}_{3} \otimes} \\
0 & \mathcal{S}_{\frac{1}{1-\mathcal{I}_{3} \otimes}}
\end{array}\right),
$$

and we have introduced

$$
\begin{aligned}
\delta \mathbf{A}_{\tilde{2}}^{\prime}= & \delta_{33} \mathbf{A}_{\tilde{2}}^{\prime}+\delta_{\tilde{2} 3} \mathbf{A}_{\tilde{2}}^{\prime} \text { and } \\
\delta \mathbf{A}_{3}^{\prime(u)}= & \mathbf{A}_{\tilde{2}}^{\prime} \frac{i \rho}{3 \omega}\left(\frac{1}{1-\mathcal{I}_{33} \otimes} \mathbf{K}_{\mathrm{df}, 33}^{(u, u)}\right)^{(u-s, u)} \\
& +\mathbf{A}_{\tilde{2}}^{\prime} \otimes \rho_{\tilde{2} 3} \otimes \frac{1}{1-\mathcal{I}_{33} \otimes} \mathbf{K}_{\mathrm{df}, 33}^{(u, u)} .
\end{aligned}
$$

Note that $\delta \mathbf{A}_{3}^{\prime(u)}$ inherits a superscript (u) from the righthand superscript of $\mathbf{K}_{\mathrm{df}, 33}^{(u, u)}$. In the following it is useful to rewrite the shifts in $\mathbf{A}^{\prime}$ as

$$
\left(\begin{array}{ll}
\delta \mathbf{A}_{\tilde{2}}^{\prime} & \delta \mathbf{A}_{3}^{\prime(u)}
\end{array}\right)=\mathbf{A}^{\prime} \otimes \mathcal{I}_{\mathbf{F L}} \otimes \mathbf{K}_{\mathrm{df}}^{(u)},
$$

where $\mathcal{I}_{\mathbf{F L}}$ is a matrix of integral operators.

The result for the $\mathbf{F}_{\mathbf{R}}$ term is given by reflection and is

$$
\mathbf{K}_{\mathrm{df}}^{(u)} \mathbf{F}_{\mathbf{R}} \mathbf{A}=\left[\mathbf{K}_{\mathrm{df}}^{(u)}\right] \mathcal{S}_{\mathbf{R}} \mathcal{F} \mathbf{A}+\left(\begin{array}{c}
\delta \mathbf{A}_{\tilde{2}} \\
\delta \mathbf{A}_{3}^{(u)}
\end{array}\right),
$$

where
$\mathbf{A}=\left(\begin{array}{c}\mathbf{A}_{\tilde{2}} \\ \mathbf{A}_{3}\end{array}\right), \quad \mathcal{S}_{\mathbf{R}}=\left(\begin{array}{cc}1 & 0 \\ \frac{1}{1-\otimes \mathcal{I}_{33}^{+}} \otimes \mathcal{I}_{3 \tilde{2}} & \frac{1}{1-\otimes \mathcal{I}_{33}^{\dagger}} \mathcal{S}\end{array}\right)$,

and $\delta \mathbf{A}_{\tilde{2}}$ and $\delta \mathbf{A}_{3}^{(u)}$ are reflections of the results in Eq. (143). Again, we introduce the matrix of integral operators $\mathcal{I}_{\mathbf{F R}}$ such that

$$
\left(\begin{array}{c}
\delta \mathbf{A}_{\tilde{2}} \\
\delta \mathbf{A}_{3}^{(u)}
\end{array}\right)=\mathbf{K}_{\mathrm{df}}^{(u)} \otimes \mathcal{I}_{\mathbf{F R}} \otimes \mathbf{A} .
$$

Finally, we turn to the symmetrization between two factors of $\mathbf{K}_{\mathrm{df}}^{(u)}$, i.e., to the analysis of

$$
\mathbf{K}_{\mathrm{df}}^{(u)}(\mathbf{X}+\mathbf{Y}) \mathbf{K}_{\mathrm{df}}^{(u)} .
$$

Only the 33 component of $(\mathbf{X}+\mathbf{Y})$ requires new work. This is because $[\mathbf{X}+\mathbf{Y}]_{\tilde{2} \tilde{2}}=\mathbf{F}_{\tilde{2} \tilde{2}}$ is already symmetrized, while, since $[\mathbf{X}+\mathbf{Y}]_{\tilde{2} 3}=\left[\mathbf{F}_{\mathbf{L}}\right]_{\tilde{2} 3}$, the analysis for the $\tilde{2} 3$ component is identical to that leading to Eq. (139), with the $3 \tilde{2}$ component given by reflection.

The contribution of the 33 component is analyzed in Appendix B 4. Combined with the results for the other components, we find that

$$
\begin{aligned}
\mathbf{K}_{\mathrm{df}}^{(u)} & (\mathbf{X}+\mathbf{Y}) \mathbf{K}_{\mathrm{df}}^{(u)} \\
= & \mathbf{K}_{\mathrm{df}}^{(u)}\left(\begin{array}{cc}
1 & 0 \\
\frac{1}{1-\otimes \mathcal{I}_{33}^{\dagger}} \otimes \mathcal{I}_{3 \tilde{2}} & \frac{1}{1-\otimes \mathcal{I}_{33}^{\dagger}} \mathcal{S}
\end{array}\right)\left(\begin{array}{ll}
\mathbf{F}_{\tilde{2} \tilde{2}} & \mathbf{F}_{\tilde{2} 3} \\
\mathbf{F}_{3 \tilde{2}} & \mathbf{F}_{33}
\end{array}\right) \\
& \times\left(\begin{array}{cc}
1 & \mathcal{I}_{\tilde{2} 3} \otimes \frac{1}{1-\mathcal{I}_{3} \otimes} \\
0 & \mathcal{S}_{\frac{1}{1-\mathcal{I}_{3} \otimes}}
\end{array}\right) \mathbf{K}_{\mathrm{df}}^{(u)}+\delta \mathbf{K}_{\mathrm{df}}^{(u)} \\
= & \mathbf{K}_{\mathrm{df}}^{(u)} \mathcal{S}_{\mathbf{R}} \mathcal{F} \mathcal{S}_{\mathbf{L}} \mathbf{K}_{\mathrm{df}}^{(u)}+\delta \mathbf{K}_{\mathrm{df}}^{(u)} .
\end{aligned}
$$

Many of the complications of the analysis are buried in the final term, $\delta \mathbf{K}_{\mathrm{df}}^{(u)}$. This arises when the two factors of $\mathbf{K}_{\mathrm{df}}^{(u)}$ 
are joined by an integral. There are several contributions to this term-those analogous to $\delta \mathbf{A}_{\tilde{2}}^{\prime}, \delta \mathbf{A}_{3}^{\prime(u)}, \delta \mathbf{A}_{\tilde{2}}$ and $\delta \mathbf{A}_{3}^{(u)}$, as well as additional terms discussed in the Appendix. For this derivation we do not require the detailed form of $\delta \mathbf{K}_{\mathrm{df}}^{(u)}$. We only require that it is composed of infinite-volume quantities, and that the symmetrization structure of its external indices is the same as that of $\mathbf{K}_{\mathrm{df}}^{(u)}$. Again, it is useful to write this term using a matrix of integral operators

$$
\delta \mathbf{K}_{\mathrm{df}}^{(u)} \equiv \mathbf{K}_{\mathrm{df}}^{(u)} \otimes \mathcal{I}_{\mathbf{X Y}} \otimes \mathbf{K}_{\mathrm{df}}^{(u)} .
$$

This emphasizes the fact that $\mathcal{I}_{\mathbf{X Y}}$ is independent of the detailed form of the quantities on either side.

We now have all the results to give a final form for the correlator. Combining Eqs. (83), (122), (141), (145) and (150), and performing straightforward but tedious algebra, we find

$$
\begin{gathered}
C_{L}^{\left[B_{2}\right]}=C_{L}^{\left[B_{2}\right],\{0\}}+C_{L}^{\left[B_{2}\right], \mathbf{K}_{\mathrm{df}}}, \\
=C_{\infty}^{\left[B_{2}\right]}+\delta C_{\infty}^{\left[B_{2}\right]}+\mathbf{A}^{\prime\left[B_{2}\right]} \mathcal{F} \frac{1}{1-\mathbf{K}_{\mathrm{df}}^{\left[B_{2}\right]} \mathcal{F}} \mathbf{A}^{\left[B_{2}\right]},
\end{gathered}
$$

where

$$
\mathbf{A}^{\prime\left[B_{2}\right]} \equiv \mathbf{A}^{\prime}+\mathbf{A}^{\prime} \otimes \mathcal{I}_{\mathbf{F L}} \otimes \mathbf{K}_{\mathrm{df}}^{(u)} \frac{1}{1-\otimes \mathcal{I}_{\mathbf{X Y}} \otimes \mathbf{K}_{\mathrm{df}}^{(u)}} \mathcal{S}_{\mathbf{R}},
$$

$$
\begin{gathered}
\mathbf{A}^{\left[B_{2}\right]} \equiv \mathbf{A}+\mathcal{S}_{\mathbf{L}} \mathbf{K}_{\mathrm{df}}^{(u)} \frac{1}{1-\otimes \mathcal{I}_{\mathbf{X Y}} \otimes \mathbf{K}_{\mathrm{df}}^{(u)}} \otimes \mathcal{I}_{\mathbf{F R}} \otimes \mathbf{A} \\
\mathbf{K}_{\mathrm{df}}^{\left[B_{2}\right]} \equiv \mathcal{S}_{\mathbf{L}} \mathbf{K}_{\mathrm{df}}^{(u)} \frac{1}{1-\otimes \mathcal{I}_{\mathbf{X Y}} \otimes \mathbf{K}_{\mathrm{df}}^{(u)}} \mathcal{S}_{\mathbf{R}}
\end{gathered}
$$$$
\delta C_{\infty}^{\left[B_{2}\right]}=\delta C_{\infty}^{\left[B_{2}\right],\{0\}}+\mathbf{A}^{\prime} \otimes \mathcal{I}_{\mathbf{F L}} \otimes \mathbf{K}_{\mathrm{df}}^{(u)} \frac{1}{1-\otimes \mathcal{I}_{\mathbf{X Y}} \otimes \mathbf{K}_{\mathrm{df}}^{(u)}}
$$$$
\otimes \mathcal{I}_{\mathbf{F R}} \otimes \mathbf{A}
$$

Equation (153) is the culmination of all the analysis contained in Secs. III B-III H, together with the corresponding Appendices, and is by far the most tedious result to derive in all our work on three-particle scattering. Having reached the very final form for all $B_{2}$-only diagrams, note that we introduce slightly more precise notation, labeling all infinite-volume quantities with the ${ }^{\left[B_{2}\right]}$ superscript to emphasize the missing $B_{3}$ kernels. In the next section we show that these are simple to incorporate.

\section{Including three-to-three kernels, $B_{3}$}

In order to complete the derivation of Eq. (38) we must include the contributions of the three-to-three kernel, $B_{3}$. This can be done by a straightforward extension of the method used in Sec. IV E of Ref. [5]. As in that work, the essential point is that the analysis described above, which takes place between end caps $\boldsymbol{\sigma}^{*}$ and $\boldsymbol{\sigma}^{\dagger *}$, applies equally well if one or both of the end caps are replaced by factors of $i B_{3} \equiv \mathbf{B}_{3}$. This is because, like $\boldsymbol{\sigma}^{*}$ and $\boldsymbol{\sigma}^{\dagger *}, \mathbf{B}_{3}$ is nonsingular in our kinematic regime. The net result is that we can reuse all the work leading to Eq. (153).

To do so we rewrite the components of Eq. (153) as

$$
\begin{aligned}
C_{\infty}^{\left[B_{2}\right]}+\delta C_{\infty}^{\left[B_{2}\right]} & \equiv \boldsymbol{\sigma}^{*} \otimes \mathcal{D}_{C} \otimes \boldsymbol{\sigma}^{\dagger *}, \\
\mathbf{A}^{\prime\left[B_{2}\right]} & \equiv \boldsymbol{\sigma}^{*} \otimes \mathcal{D}_{\mathbf{A}^{\prime}}, \\
\mathbf{A}^{\left[B_{2}\right]} & \equiv \mathcal{D}_{\mathbf{A}} \otimes \boldsymbol{\sigma}^{\dagger *}, \\
\mathcal{Z} & \equiv \mathcal{F} \frac{1}{1-\mathbf{K}_{\mathrm{df}}^{\left[B_{2}\right]} \mathcal{F}},
\end{aligned}
$$

in terms of which

$$
C_{L}^{\left[B_{2}\right]}=\boldsymbol{\sigma}^{*} \otimes\left\{\mathcal{D}_{C}+\mathcal{D}_{\mathbf{A}^{\prime}} \mathcal{Z} \mathcal{D}_{\mathbf{A}}\right\} \otimes \boldsymbol{\sigma}^{\dagger *} .
$$

Here $\mathcal{D}_{C}, \mathcal{D}_{\mathbf{A}^{\prime}}$ and $\mathcal{D}_{\mathbf{A}}$ are infinite-volume decoration operators that contain the complicated contributions worked out above. ${ }^{16}$ Note that $\mathcal{D}_{\mathbf{A}}$ and $\mathcal{D}_{\mathbf{A}^{\prime}}$ are, respectively, $2 \times 1$ and $1 \times 2$ matrices. All we need to know in this section is that the decoration operators are well defined, and apply just as well when the end caps are replaced by factors of $\mathbf{B}_{3}$.

The full finite-volume correlator, including all possible $B_{2}$ and $B_{3}$ insertions, can now be written

$$
\begin{aligned}
C_{L}= & \boldsymbol{\sigma}^{*} \otimes\left\{\mathcal{D}_{C}+\mathcal{D}_{\mathbf{A}^{\prime}} \mathcal{Z D}_{\mathbf{A}}\right\} \\
& \otimes \sum_{n=0}^{\infty}\left(\mathbf{B}_{3} \otimes\left\{\mathcal{D}_{C}+\mathcal{D}_{\mathbf{A}^{\prime}} \mathcal{Z D}_{\mathbf{A}}\right\} \otimes\right)^{n} \boldsymbol{\sigma}^{\dagger *} .
\end{aligned}
$$

Rearranging the series in powers of $\mathcal{Z}$ we find

$$
C_{L}=C_{\infty}+\sum_{n=0}^{\infty} \mathbf{A}^{\prime} \mathcal{Z}\left(\mathbf{K}_{\mathrm{df}}^{\left[B_{3}\right]} \mathcal{Z}\right)^{n} \mathbf{A}
$$

where we have defined the infinite-volume quantities

$$
\begin{array}{r}
C_{\infty} \equiv \boldsymbol{\sigma}^{*} \otimes \mathcal{D}_{C} \otimes \sum_{n=0}^{\infty}\left(\mathbf{B}_{3} \otimes \mathcal{D}_{C} \otimes\right)^{n} \boldsymbol{\sigma}^{\dagger *}, \\
\mathbf{A}^{\prime} \equiv \boldsymbol{\sigma}^{*} \otimes \sum_{n=0}^{\infty}\left(\mathcal{D}_{C} \otimes \mathbf{B}_{3} \otimes\right)^{n} \mathcal{D}_{\mathbf{A}^{\prime}},
\end{array}
$$

\footnotetext{
${ }^{16}$ In Ref. [5] the corresponding decoration operators were given superscripts, but here we drop these for the sake of brevity.
} 


$$
\begin{gathered}
\mathbf{A}=\mathcal{D}_{\mathbf{A}} \otimes \sum_{n=0}^{\infty}\left(\mathbf{B}_{3} \otimes \mathcal{D}_{C} \otimes\right)^{n} \boldsymbol{\sigma}^{*} \\
\mathbf{K}_{\mathrm{df}}^{\left[B_{3}\right]} \equiv \mathcal{D}_{\mathbf{A}} \otimes \mathbf{B}_{3} \otimes \sum_{n=0}^{\infty}\left(\mathcal{D}_{C} \otimes \mathbf{B}_{3} \otimes\right)^{n} \mathcal{D}_{\mathbf{A}^{\prime}}
\end{gathered}
$$

Inserting the definition of $\mathcal{Z}$, Eq. (161), into the result for $C_{L}$, and rearranging, we reach the final form given in Eq. (38) above. In terms of our boldface quantities it reads

$$
C_{L}=C_{\infty}+\mathbf{A}^{\prime} \mathcal{F} \frac{1}{1-\mathbf{K}_{\mathrm{df}} \mathcal{F}} \mathbf{A}
$$

where

$$
\mathbf{K}_{\mathrm{df}} \equiv \mathbf{K}_{\mathrm{df}}^{\left[B_{2}\right]}+\mathbf{K}_{\mathrm{df}}^{\left[B_{3}\right]} .
$$

\section{RELATING $\mathcal{K}_{\text {df }}$ TO THE THREE-PARTICLE SCATTERING AMPLITUDE}

Having completed the derivation of the quantization condition, i.e., the relation between the finite-volume spectrum and $\mathcal{K}_{\mathrm{df}}$, we now turn to relating the latter to the physical three-to-three scattering amplitude, $\mathcal{M}_{3}$. Following Ref. [6], we derive equations relating $\mathcal{K}_{\mathrm{df}}$ to $\mathcal{M}_{3}$ in two steps. First, in Sec. IV A we give a modified version of our main result, Eq. (38), in terms of a new finitevolume correlator, denoted $\mathcal{M}_{L, 3}$. Second, in Sec. IV B, we analytically study a carefully defined $L \rightarrow \infty$ limit in which $\mathcal{M}_{L, 3} \rightarrow \mathcal{M}_{3}$. The result is a series of integral equations relating the divergence-free $\mathrm{K}$ matrix to the scattering amplitude. In this section we return to the notation of Sec. II in which factors of $i$ and $1 /\left(2 \omega L^{3}\right)$ are displayed explicitly.

\section{A. Relating $\mathcal{K}_{\mathrm{df}}$ to $\mathcal{M}_{L, 3}$}

In order to relate the components of $\mathcal{K}_{\mathrm{df}}$ to physical quantities, we need to determine the volume-dependence of $\mathcal{M}_{L, 3}$, first introduced in Ref. [6]. $\mathcal{M}_{L, 3}$ differs from $C_{L}$ in two ways. First, the diagrams have three on-shell, amputated propagators on each end, rather than the generic operators $\mathcal{O}(x)$ and $\mathcal{O}^{\dagger}(x)$ included in Eq. (37). Second, we allow the momenta of these external particles to be arbitrary, and not constrained to lie in the finite-volume set. As discussed at length in Ref. [6], the latter property is necessary in order to take the infinite-volume limit. Despite these differences we argue here that we can obtain the result for $\mathcal{M}_{L, 3}$ from that for $C_{L}$, Eq. (38).

We rely on several key observations from Ref. [6], where, we recall, $\mathcal{M}_{L, 3}$ was analyzed for systems without poles in $\mathcal{K}_{2}$. The first is that $C_{L}$ contains all the diagrams contributing to $\mathcal{M}_{L, 3}$. The task is to separate these out. In particular, we need contributions in which three particles are on shell, rather than part of an unconstrained loop sum.
The second observation is that, in the final form for $C_{L}$, onshell three-particle states occur whenever there is a factor of $F$ or $G$. In particular, if we take the expression for $C_{L}$ and restrict attention to terms with at least two $F$ or $G$ cuts, then the expression lying between the outermost such cuts will contain all contributions to $\mathcal{M}_{L, 3}$. It will turn out that the outermost cuts are always factors of $F$ rather than $G$. The third observation is that amputation is effected by removing the external factors of $i F$ and multiplying by $2 \omega L^{3}$ on the left and right. After doing so, the result is equal to $\mathcal{M}_{L, 3}$ aside from two final adjustments. The first is to drop disconnected contributions, and the second is to symmetrize. We discuss these two relatively minor steps in more detail below.

In fact, Ref. [6] did not apply these observations to the final result for $C_{L}$, but rather to an intermediate result. Additional analysis was then required to obtain the final expression for $\mathcal{M}_{L, 3}$. It was noted that the result for $\mathcal{M}_{L, 3}$ could have been obtained by applying the amputation procedure directly to the decomposition of $C_{L}$, but it was argued that this was a mnemonic rather than a rigorous procedure (see footnote 10 of Ref. [6]). We now think, however, that inferring the form of $\mathcal{M}_{L, 3}$ from $C_{L}$, by directly converting the final result is justified, and indeed that the work of Ref. [6] supports this claim. We explain additional justification for this new approach below, once we have obtained the result for $\mathcal{M}_{L, 3}$.

Due to the presence of poles in $\mathcal{K}_{2}$, the procedure described above must be amended. To understand the issue, we focus on the contribution to $C_{L}$ arising from a single insertion of the $3 \tilde{2}$ component of $\mathcal{F}$, namely

$$
i A_{3}^{\prime} i F_{3 \tilde{2}} i A_{\tilde{2}}=i A_{3}^{\prime} \frac{1}{2 \omega L^{3}} i F \frac{1}{1-i \mathcal{K}_{2}(i F+i G)} i \Gamma_{J} i G_{\rho}^{\dagger} i A_{\tilde{2}},
$$

$$
\begin{aligned}
= & i A_{3}^{\prime} \frac{1}{2 \omega L^{3}} i F i \Gamma_{J} i G_{\rho}^{\dagger} i A_{\tilde{2}} \\
& +i A_{3}^{\prime} \frac{1}{2 \omega L^{3}} i F i \mathcal{K}_{2}(i F+i G) i \Gamma_{J} i G_{\rho}^{\dagger} i A_{\tilde{2}} \\
& +i A_{3}^{\prime} \frac{1}{2 \omega L^{3}} i F i \mathcal{K}_{2}(i F+i G) i \mathcal{K}_{2}(i F+i G) i \Gamma_{J} i G_{\rho}^{\dagger} i A_{\tilde{2}}+\cdots
\end{aligned}
$$

The first term in Eq. (172) can be dropped as it has only one $F$ or $G$ cut. The second term has two such cuts, but only a single $\mathcal{K}_{2}$ lies between them, so this corresponds to a disconnected contribution to $\mathcal{M}_{L, 3}$. Thus this term is also dropped. The third term has two external cuts, and part of the contribution between them is connected, namely the $i \mathcal{K}_{2} i G i \mathcal{K}_{2}$ part. However, such a contribution is already contained in the $i A_{3}^{\prime} i F_{33} i A_{3}$ term, as is readily checked. A signal for this double counting is that there is a $\rho \pi$ cut, $G_{\rho}^{\dagger}$, that is external relative to the right-hand $F / G$-cut in 
each of the terms in Eq. (172). Indeed, one can show that the complete set of contributions to $\mathcal{M}_{L, 3}$ is obtained by taking only terms in which the outermost cut contains three particles rather than the $\rho \pi$ effective channel. This extra criterion implies that none of the terms in Eq. (172) should be kept.

The same conclusion holds for single insertions of $F_{\tilde{2} 3}$ or $F_{\tilde{2} \tilde{2}}$, which have, respectively, one and two external $\rho \pi$ cuts. Thus the only surviving contribution from a single insertion of $\mathcal{F}$ is that from $F_{33}$. This contribution is unaffected by the presence of poles in $\mathcal{K}_{2}$, and so is unchanged from that obtained in Ref. [6]. We recall briefly how this term is obtained. Using the result for $F_{33}$, Eq. (18), we find that the term with at least two three-particle cuts is

$$
C_{L} \supset i A_{3}^{\prime} i F_{33} i A_{3} \supset i A_{3}^{\prime} \frac{1}{2 \omega L^{3}} i F \frac{1}{1-i \mathcal{K}_{2}(i F+i G)} i \mathcal{K}_{2} i F i A_{3} .
$$

Applying the recipe given above we obtain

$\left.i \mathcal{M}_{L, 3} \supset\left\{\frac{1}{1-i \mathcal{K}_{2}(i F+i G)} i \mathcal{K}_{2}\left[2 \omega L^{3}\right]\right\}\right|_{\text {connected,symmetrized }}$.

The disconnected part is that obtained by setting $G \rightarrow 0$. We can remove this, and at the same time make contact with the notation of Ref. [6], using the identity

$$
\frac{1}{1-i \mathcal{K}_{2}(i F+i G)} i \mathcal{K}_{2}\left[2 \omega L^{3}\right]=i \mathcal{M}_{L, 2}\left[2 \omega L^{3}\right]+i \mathcal{D}_{L}^{(u, u)},
$$

where

$$
\begin{gathered}
i \mathcal{M}_{L, 2}=\frac{1}{1-i \mathcal{K}_{2} i F} i \mathcal{K}_{2}, \\
i \mathcal{D}_{L}^{(u, u)}=\frac{1}{1-i \mathcal{M}_{2, L} i G} i \mathcal{M}_{L, 2} i G i \mathcal{M}_{L, 2}\left[2 \omega L^{3}\right] .
\end{gathered}
$$

The first term in Eq. (175) contains no switches and thus leads to a disconnected contribution. The second term contains at least one switch and thus is connected; it agrees with the quantity of the same name given in Eq. (25) of Ref. [6]. Thus we find

$$
\left.i \mathcal{M}_{L, 3} \supset\left\{i \mathcal{D}_{L, 2}^{(u, u)}\right\}\right|_{\text {symmetrized }} \equiv \mathcal{S i D}_{L, 2}^{(u, u)} \mathcal{S} .
$$

We can now explain the need for symmetrization. In the original expression, $i A_{3}^{\prime} i F_{33} i A_{3}$, the end caps $A_{3}^{\prime}$ and $A_{3}$ are fully symmetrized, as described earlier in the derivation. By this we mean that the quantities are invariant under interchange of any of the three-particle momenta. The adjacent factors of $F$ that will be removed are not, however, symmetric, since they single out one of the on-shell particles as the spectator. Similarly, what lies between the two amputated $F \mathrm{~s}$ is not symmetric. Within $C_{L}$ this does not matter, because of the symmetry of the end caps. But $\mathcal{M}_{L, 3}$ is defined to be symmetric on the external lines, and to reproduce this we must sum over all permutations of the three incoming and three outgoing particle momenta. However, it turns out that all quantities entering these expressions are already invariant under interchange of the two nonspectators, so that one need only sum over the remaining three distinct permutations. The precise action of the symmetrization operators is described by the paragraph containing Eqs. (35)-(37) in Ref. [6]. ${ }^{17}$

Now we apply the updated rules to the terms in Eq. (38) having more than one factor of $\mathcal{F}$, namely

$$
C_{L} \supset i A^{\prime} i \mathcal{F} \frac{1}{1-i \mathcal{K}_{\mathrm{df}} i \mathcal{F}} i \mathcal{K}_{\mathrm{df}} i \mathcal{F} i A
$$

Since we are requiring an external three-particle cut rather than a $\rho \pi$ cut, only $F_{33}$ and $F_{32}$ contribute from the lefthand $\mathcal{F}$, and only $F_{33}$ and $F_{\tilde{2} 3}$ contribute from the righthand $\mathcal{F}$. Thus we find that the contribution to $\mathcal{M}_{L, 3}$ is

$$
\begin{gathered}
i \mathcal{M}_{L, 3} \supset \mathcal{S} \mathcal{L}_{L}^{(u)} \frac{1}{1-i \mathcal{K}_{\mathrm{df}} i \mathcal{F}} i \mathcal{K}_{\mathrm{df}} \mathcal{R}_{L}^{(u)} \mathcal{S}, \\
\mathcal{L}_{L}^{(u)}=\left(\frac{1}{1-i \mathcal{K}_{2}(i F+i G)} i \Gamma_{J} i G_{\rho}^{\dagger}\right. \\
\left.\frac{1}{3}+\frac{1}{1-i \mathcal{K}_{2}(i F+i G)} i \mathcal{K}_{2} i F\right),
\end{gathered}
$$

$$
\mathcal{R}_{L}^{(u)}=\left(\begin{array}{c}
i G_{\rho} i \Gamma_{J} \frac{1}{1-\left(i F+i G^{\dagger}\right) i \mathcal{K}_{2}} \\
\frac{1}{3}+i F i \mathcal{K}_{2} \frac{1}{1-\left(i F+i G^{\dagger}\right) i \mathcal{K}_{2}}
\end{array}\right) .
$$

Here $G^{\dagger}=\left[2 \omega L^{3}\right]^{-1} G\left[2 \omega L^{3}\right]$, as follows from the definition of the matrix $G$, Eq. (21). Combining this result with that from Eq. (178) leads to the full expression for $\mathcal{M}_{L, 3}$,

$\mathcal{M}_{L, 3}=\mathcal{S}\left\{\mathcal{D}_{L}^{(u, u)}+\mathcal{L}_{L}^{(u)} \mathcal{K}_{\mathrm{df}} \frac{1}{1+\mathcal{F} \mathcal{K}_{\mathrm{df}}} \mathcal{R}_{L}^{(u)}\right\} \mathcal{S}$.

Here we have multiplied various factors of $i$ together and divided both sides of the equation by $i$. We stress again that no factors of $i$ or $2 \omega L^{3}$ have been absorbed here by redefinitions.

A consistency check on this derivation is that the external factors that are amputated to obtain $\mathcal{M}_{L, 3}$ from $C_{L}$ are the

\footnotetext{
${ }^{17}$ Note that, in that work, the symmetrization operators acting to the right and left are packaged into a single overall symmetrization operator.
} 
same for both Eqs. (178) and (180), namely $i A_{3}^{\prime} \frac{1}{2 \omega L^{3}} i F$ on the left and $i F \frac{1}{2 \omega L^{3}} i A_{3}$ on the right. ${ }^{18}$

Finally, we return to the issue of why we now think the above procedure for obtaining $\mathcal{M}_{L, 3}$ from $C_{L}$ is valid. We raised two concerns in Ref. [6]. The first was essentially that the infinite-volume quantities appearing in $C_{L}$ resulted from a sequence of redefinitions, obscuring the relation to the underlying diagrams. Here we have been able to give a more explicit form for these redefinitions, i.e., those in Eqs. (154)-(156), (166), (167) and (170). This gives us confidence that there are no subtleties in picking out the parts of the diagrams that contribute to $\mathcal{M}_{L, 3}$. The second concern was that the symmetrization procedure after amputation was not justified. We have now convinced ourselves, as described above, that it is correct.

\section{B. Applying the formal $L \rightarrow \infty$ limit to relate $\mathcal{K}_{\mathrm{df}}$ to the three-to-three scattering amplitude}

We are now ready to apply the $L \rightarrow \infty$ limit to Eq. (183), and thereby derive an integral equation relating $\mathcal{K}_{\mathrm{df}}$ to the physical three-to-three scattering amplitude, $\mathcal{M}_{3}$. We begin by recalling the expression for $\mathcal{F}$ [Eqs. (3), (18), (29), (30) and (31)],

$$
\begin{gathered}
\mathcal{F}=\left(\begin{array}{ll}
F_{\tilde{2} \tilde{2}} & F_{\tilde{2} 3} \\
F_{3 \tilde{2}} & F_{33}
\end{array}\right) \\
F_{\tilde{2} \tilde{2}} \equiv F_{\rho \pi}+G_{\rho} \Gamma_{J} \frac{1}{2 \omega L^{3}}(F+G) \frac{1}{1+\mathcal{K}_{2}(F+G)} \Gamma_{J} G_{\rho}^{\dagger}, \\
F_{\tilde{2} 3} \equiv-G_{\rho} \Gamma_{J} \frac{1}{2 \omega L^{3}} \frac{1}{1+(F+G) \mathcal{K}_{2}} F \\
F_{3 \tilde{2}} \equiv-\frac{1}{2 \omega L^{3}} F \frac{1}{1+\mathcal{K}_{2}(F+G)} \Gamma_{J} G_{\rho}^{\dagger} \\
F_{33}=\frac{1}{2 \omega L^{3}}\left[\frac{F}{3}-F \frac{1}{1+\mathcal{K}_{2}(F+G)} \mathcal{K}_{2} F\right]
\end{gathered}
$$

Here we have again combined various factors of $i$ to simplify the expressions.

The method we use is that developed in Ref. [6]. We want to take $L \rightarrow \infty$ in such a way that $\mathcal{M}_{L, 3}$ goes over to $\mathcal{M}_{3}$. This requires that we first regularize poles in integrands with the ie prescription, and then take the $L \rightarrow \infty$ limit with $\epsilon$ held fixed. As explained in Ref. [6], this limit sends $F \rightarrow \rho$ (since $F^{i \epsilon} \rightarrow 0$ ), and $\mathcal{M}_{L, 2} \rightarrow \mathcal{M}_{2}$. Matrix products, combined with factors of $1 / L^{3}$, go over to integrals. We also need to introduce $G^{\infty}$, defined by

\footnotetext{
${ }^{18}$ Note that the matrices $\frac{1}{2 \omega L^{3}}$ and $F$ commute, though neither commutes with $G$.
}

$G_{\ell^{\prime} m^{\prime}, \ell m}^{\infty}(\vec{p}, \vec{k})=\mathcal{Y}_{3, \ell^{\prime} m^{\prime}}\left(\vec{k}_{2, p}^{*}\right) \mathbf{S}_{3}^{i \epsilon}(\vec{p}, \vec{k}) \mathcal{Y}_{3, \ell m}^{*}\left(\vec{p}_{2, k}^{*}\right)$

In Ref. [6], the only poles present were the three-particle poles in $\mathbf{S}_{3}$. Here we also have the possibility of K-matrix poles, which are present in $G_{\rho}$ and $F_{\rho \pi}$ as well as in $\mathcal{K}_{2}$ itself. However, we know that K-matrix poles cannot be present in $\mathcal{M}_{3}$, because poles on the real axis of scattering amplitudes would imply a violation of unitarity. In fact, we show that they are absent also in $\mathcal{M}_{L, 3}$, so that there is no need to regularize them.

To see the absence of K-matrix poles we begin by rewriting Eq. (182) as

$$
\mathcal{R}_{L}^{(u)}=\left(\begin{array}{c}
-G_{\rho} \Gamma_{J} \frac{1}{1+F \mathcal{K}_{2}} \\
\frac{1}{3}-F \mathcal{M}_{L, 2}
\end{array}\right) \frac{1}{1+G^{\dagger} \mathcal{M}_{L, 2}} .
$$

Here we recall that

$$
\mathcal{M}_{L, 2}=\frac{1}{1+\mathcal{K}_{2} F} \mathcal{K}_{2}=\frac{1}{\mathcal{K}_{2}^{-1}+F}
$$

which shows explicitly that poles in $\mathcal{K}_{2}$ do not lead to poles in $\mathcal{M}_{L, 2}$. The same cancellation occurs for the poles in $G_{\rho}$,

$$
-G_{\rho} \Gamma_{J} \frac{1}{1+F \mathcal{K}_{2}}=-G_{\rho} \Gamma_{J} \mathcal{K}_{2}^{-1} \mathcal{M}_{L, 2}
$$

Since $\Gamma_{J}$ is a constant, $G_{\rho} \Gamma_{J} \mathcal{K}_{2}^{-1}$ is smooth at the pole position. It is also a known quantity, assuming that we know $\mathcal{K}_{2}$ from the two-particle quantization condition, and has a well-defined infinite-volume limit.

We can similarly rewrite the other quantities involving $G_{\rho}$ (or its Hermitian conjugate) in such a way that they are manifestly free of K-matrix poles,

$$
\mathcal{L}_{L}^{(u)}=\frac{1}{1+\mathcal{M}_{L, 2} G}\left(-\mathcal{M}_{L, 2} \mathcal{K}_{2}^{-1} \Gamma_{J} G_{\rho}^{\dagger} \quad \frac{1}{3}-\mathcal{M}_{L, 2} F\right),
$$

$$
\begin{gathered}
F_{\tilde{2} 3}=-G_{\rho} \Gamma_{J} \mathcal{K}_{2}^{-1} \mathcal{M}_{L, 2} \frac{1}{1+G^{\dagger} \mathcal{M}_{L, 2}} \frac{1}{2 \omega L^{3}} F \\
=-G_{\rho} \Gamma_{J} \mathcal{K}_{2}^{-1} \frac{1}{2 \omega L^{3}} \frac{1}{1+\mathcal{M}_{L, 2} G} \mathcal{M}_{L, 2} F, \\
F_{3 \tilde{2}}=-\frac{1}{2 \omega L^{3}} F \frac{1}{1+\mathcal{M}_{L, 2} G} \mathcal{M}_{L, 2} \mathcal{K}_{2}^{-1} \Gamma_{J} G_{\rho}^{\dagger} .
\end{gathered}
$$

This leaves $F_{\tilde{2} \tilde{2}}$, which contains $F_{\rho \pi}$. This can be rewritten as 


$$
\begin{aligned}
F_{\tilde{2} \tilde{2}}= & -G_{\rho} \Gamma_{J} \mathcal{K}_{2}^{-1} \frac{1}{2 \omega L^{3}} \frac{1}{1+\mathcal{M}_{L, 2} G} \mathcal{M}_{L, 2} \mathcal{K}_{2}^{-1} \Gamma_{J} G_{\rho}^{\dagger} \\
& +\left\{F_{\rho \pi}+G_{\rho} \Gamma_{J} \frac{1}{2 \omega L^{3}} \mathcal{K}_{2}^{-1} \Gamma_{J} G_{\rho}^{\dagger}\right\} .
\end{aligned}
$$

The first term is manifestly free of K-matrix poles. For the term in curly braces, the poles also cancel. To see this we note that $F_{\rho \pi}$ contains a sum over spectator momenta, which is matched in the $G_{\rho}[\cdots] G_{\rho}^{\dagger}$ part by the sum over matrix indices. The infinite-volume limit of this term is known given knowledge of $\mathcal{K}_{2}$.

The final quantity to be considered is $F_{33}$. Here the absence of K-matrix poles is manifest, but it is still useful to rewrite it as

$$
F_{33}=\frac{1}{2 \omega L^{3}} F\left[\frac{1}{3}-\frac{1}{1+\mathcal{M}_{L, 2} G} \mathcal{M}_{L, 2} F\right] .
$$

It is now a tedious but straightforward exercise to take the infinite-volume limit of Eq. (183). We first introduce useful infinite-volume quantities

$$
\begin{gathered}
G_{\rho} \Gamma_{J} \mathcal{K}_{2}^{-1} \stackrel{L \rightarrow \infty}{\longrightarrow} \bar{G}_{\rho ; M_{J}^{\prime} \ell^{\prime} m^{\prime} ; \ell m}(\vec{k}), \\
\mathcal{K}_{2}^{-1} \Gamma_{J} G_{\rho}^{\dagger} \stackrel{L \rightarrow \infty}{\longrightarrow} \bar{G}_{\rho ; \ell m, M_{J} \ell^{\prime} m^{\prime}}^{\dagger}(\vec{k}), \\
\left\{F_{\rho \pi}+G_{\rho} \Gamma_{J} \frac{1}{2 \omega L^{3}} \mathcal{K}_{2}^{-1} \Gamma_{J} G_{\rho}^{\dagger}\right\} \stackrel{L \rightarrow \infty}{\longrightarrow} \bar{F}_{\rho \pi ; M_{J}^{\prime} \ell^{\prime} m^{\prime} ; M_{J} \ell m} .
\end{gathered}
$$

We note that these quantities contain information about the spin of the resonance; for example, $\bar{G}_{\rho}$ contains a factor of $\delta_{J \ell}$. All three quantities are determined by $\mathcal{K}_{2}$.

The matrix $\left[1+\mathcal{M}_{2, L} G\right]^{-1}$ occurs repeatedly. In the $L \rightarrow \infty$ limit, multiplication by this matrix is replaced by integration with the $\mathcal{U}(\vec{p}, \vec{k})_{\ell^{\prime} m^{\prime} ; \ell m}$, which solves the integral equation

$$
\begin{aligned}
\mathcal{U}(\vec{p}, \vec{k})= & (2 \pi)^{3} \delta^{3}(\vec{p}-\vec{k}) \\
& -\int_{s} \mathcal{M}_{2}(\vec{p}) G^{\infty}(\vec{p}, \vec{s}) \frac{1}{2 \omega_{s}} \mathcal{U}(\vec{s}, \vec{k}) .
\end{aligned}
$$

Here $\int_{s} \equiv \int d^{3} s /(2 \pi)^{3}$, and we are keeping the angularmomentum indices implicit.

We next construct the infinite-volume limits of the elements of $\mathcal{F}$. Pulling out overall factors of $1 / L^{3}$ that will turn sums into integrals, we find that these limits give

$$
\begin{gathered}
\bar{F}_{\tilde{2} \tilde{2}}=-\int_{s} \int_{t} \bar{G}_{\rho}(\vec{s}) \frac{1}{2 \omega_{s}} \mathcal{U}(\vec{s}, \vec{t}) \mathcal{M}_{2}(\vec{t}) \bar{G}_{\rho}^{\dagger}(\vec{t})+\bar{F}_{\rho \pi} \\
\bar{F}_{\tilde{2} 3}(\vec{k})=-\int_{s} \frac{1}{2 \omega_{s}} \bar{G}_{\rho}(\vec{s}) \mathcal{U}(\vec{s}, \vec{k}) \mathcal{M}_{2}(\vec{k}) \rho(\vec{k}),
\end{gathered}
$$

$$
\begin{gathered}
\bar{F}_{3 \tilde{2}}(\vec{p})=-\frac{\rho(\vec{p})}{2 \omega_{p}} \int_{s} \mathcal{U}(\vec{p}, \vec{s}) \mathcal{M}_{2}(\vec{s}) \bar{G}_{\rho}^{\dagger}(\vec{s}), \\
\bar{F}_{33}(\vec{p}, \vec{k})=\frac{\rho(\vec{p})}{6 \omega_{p}}(2 \pi)^{3} \delta^{3}(\vec{p}-\vec{k})-\frac{\rho(\vec{p})}{2 \omega_{p}} \mathcal{U}(\vec{p}, \vec{k}) \mathcal{M}_{2}(\vec{k}) \rho(\vec{k}) .
\end{gathered}
$$

All these quantities can be determined given knowledge of $\mathcal{M}_{2}$. We also recall that $\rho(\vec{k})$ contains the cutoff function $H(\vec{k})$, so that all integrals have finite range.

The next stage is to determine the limit of $\mathcal{K}_{\text {df }}\left(1+\mathcal{F} \mathcal{K}_{\text {df }}\right)^{-1}$, which we call $\mathcal{T}$. This leads to two pairs of coupled matrix-integral equations for the components of $\mathcal{T}$. The first pair is

$$
\begin{aligned}
\mathcal{T}_{\tilde{2} \tilde{2}}= & \mathcal{K}_{\mathrm{df}, \tilde{2} \tilde{2}}-\mathcal{K}_{\mathrm{df}, \tilde{2} \tilde{2}} \bar{F}_{\tilde{2} \tilde{2}} \mathcal{T}_{\tilde{2} \tilde{2}}-\int_{t} \mathcal{K}_{\mathrm{df}, \tilde{2} \tilde{2}} \bar{F}_{\tilde{2} 3}(\vec{t}) \mathcal{T}_{3 \tilde{2}}(\vec{t}) \\
& -\int_{s} \mathcal{K}_{\mathrm{df}, \tilde{2} 3}(\vec{s}) \bar{F}_{3 \tilde{2}}(\vec{s}) \mathcal{T}_{\tilde{2} \tilde{2}} \\
& -\int_{s, t} \mathcal{K}_{\mathrm{df}, \tilde{2} 3}(\vec{s}) \bar{F}_{33}(\vec{s}, \vec{t}) \mathcal{T}_{3 \tilde{2}}(\vec{t}) \\
\mathcal{T}_{3 \tilde{2}}(\vec{p})= & \mathcal{K}_{\mathrm{df}, 3 \tilde{2}}(\vec{p})-\mathcal{K}_{\mathrm{df}, 3 \tilde{2}}(\vec{p}) \bar{F}_{\tilde{2} \tilde{2}} \mathcal{T}_{\tilde{2} \tilde{2}} \\
& -\int_{t} \mathcal{K}_{\mathrm{df}, 3 \tilde{2}}(\vec{p}) \bar{F}_{\tilde{2} 3}(\vec{t}) \mathcal{T}_{3 \tilde{2}}(\vec{t}) \\
& -\int_{s} \mathcal{K}_{\mathrm{df}, 33}(\vec{p}, \vec{s}) \bar{F}_{3 \tilde{2}}(\vec{s}) \mathcal{T}_{\tilde{2} \tilde{2}} \\
& -\int_{s, t} \mathcal{K}_{\mathrm{df}, 33}(\vec{p}, \vec{s}) \bar{F}_{33}(\vec{s}, \vec{t}) \mathcal{T}_{3 \tilde{2}}(\vec{t})
\end{aligned}
$$

The second pair is a straightforward generalization given by replacing all rightmost 2 indices with 3 indices and including the appropriate additional momentum dependencies.

Finally, given $\mathcal{T}$ we can obtain $\mathcal{M}_{3}$ by doing integrals. The contribution of $\mathcal{D}_{L}^{(u, u)}$ is unchanged from Ref. [6]. We obtain it using

$\mathcal{D}^{(u, u)}(\vec{p}, \vec{k})=-\int_{s} \mathcal{U}(\vec{p}, \vec{s}) \mathcal{M}_{2}(\vec{s}) G^{\infty}(\vec{s}, \vec{k}) \mathcal{M}_{2}(\vec{k})$.

For the remaining term we multiply $\mathcal{T}$ on the left with

$$
\begin{aligned}
& \overline{\mathcal{L}}_{\infty}^{(u)}(\vec{p}, \vec{s}) \\
& \quad \equiv\left(-\mathcal{U}(\vec{p}, \vec{s}) \mathcal{M}_{2}(\vec{s}) \bar{G}_{\rho}(\vec{s}), \mathcal{U}(\vec{p}, \vec{s})\left[\frac{1}{3}-\mathcal{M}_{2}(\vec{s}) \rho(\vec{s})\right]\right),
\end{aligned}
$$

and integrate the $\vec{s}$ coordinate. Similarly we multiply with the conjugate, $\overline{\mathcal{R}}_{\infty}^{(u)}(\vec{t}, \vec{k})$, on the right and integrate again to reach 


$$
\begin{aligned}
& \mathcal{M}_{3}^{(u, u)}(\vec{p}, \vec{k}) \\
& =\mathcal{D}^{(u, u)}(\vec{p}, \vec{k})+\int_{s, t} \overline{\mathcal{L}}_{\infty}^{(u)}(\vec{p}, \vec{s}) \mathcal{T}(\vec{s}, \vec{t}) \overline{\mathcal{R}}_{\infty}^{(u)}(\vec{t}, \vec{k}) .
\end{aligned}
$$

This result can then be converted to a function of the three incoming and three outgoing momenta via

$$
\begin{aligned}
& \mathcal{M}_{3}^{(u, u)}\left(\vec{p}, \vec{a}^{\prime}, \vec{b}^{\prime} ; \vec{k}, \vec{a}, \vec{b}\right) \\
& \quad \equiv 4 \pi Y_{\ell^{\prime} m^{\prime}}\left(\hat{a}_{2, p}^{\prime *}\right) \mathcal{M}_{3 ; \ell^{\prime} m^{\prime} ; \ell m}^{(u, u)}(\vec{p}, \vec{k}) Y_{\ell m}^{*}\left(\hat{a}_{2, k}^{*}\right),
\end{aligned}
$$

where $\vec{b}^{\prime} \equiv \vec{P}-\vec{p}-\vec{a}^{\prime}$ and $\vec{b} \equiv \vec{P}-\vec{k}-\vec{a}$, and we have restored the angular-momentum indices on $\mathcal{M}_{3}^{(u, u)}$ on the right-hand side. Finally, the physical scattering amplitude is reached by symmetrizing

$$
\begin{aligned}
& \mathcal{M}_{3}\left(\vec{p}, \vec{a}^{\prime}, \vec{b}^{\prime} ; \vec{k}, \vec{a}, \vec{b}\right) \\
& =\mathcal{S}\left[\mathcal{M}_{3}^{(u, u)}\right] \mathcal{S} \\
& \equiv \sum_{\vec{p}_{1}, \vec{p}_{2}, \vec{p}_{3} \in \mathcal{P}_{\vec{p}} \sum_{\vec{k}_{1}, \vec{k}_{2}, \vec{k}_{3} \in \mathcal{P}_{\vec{k}}} \mathcal{M}_{3}^{(u, u)}\left(\vec{p}_{1}, \vec{p}_{2}, \vec{p}_{3} ; \vec{k}_{1}, \vec{k}_{2}, \vec{k}_{3}\right),}
\end{aligned}
$$

where

$$
\begin{aligned}
& \mathcal{P}_{\vec{p}} \equiv\left\{\left\{\vec{p}, \vec{a}^{\prime}, \vec{b}^{\prime}\right\},\left\{\vec{b}^{\prime}, \vec{p}, \vec{a}^{\prime}\right\},\left\{\vec{a}^{\prime}, \vec{b}^{\prime}, \vec{p}\right\}\right\}, \quad \text { and } \\
& \mathcal{P}_{\vec{k}} \equiv\{\{\vec{k}, \vec{a}, \vec{b}\},\{\vec{b}, \vec{k}, \vec{a}\},\{\vec{a}, \vec{b}, \vec{k}\}\} .
\end{aligned}
$$

\section{CONCLUSION}

In this work we have lifted the final major restriction on our formalism relating finite-volume energies to relativistic two- and three-particle scattering amplitudes. To summarize, at this stage we have the building blocks to treat any system of identical scalar particles. Our results fall into the following three classes:

(1) $\mathbf{3} \rightarrow \mathbf{3}$ scattering assuming a $\mathbb{Z}_{2}$ symmetry and no subchannel resonances (i.e., no poles in $\mathcal{K}_{2}$; see Refs. [5,6]),

(2) $\{\mathbf{2}, \mathbf{3}\} \rightarrow\{\mathbf{2}, \mathbf{3}\}$ scattering in the case of no $\mathbb{Z}_{2}$ symmetry and, again, no subchannel resonances (see Ref. [7]),

(3) $\mathbf{3} \rightarrow \mathbf{3}$ scattering for systems with a pole in $\mathcal{K}_{2}$ (this work).

To complete the formalism for all two- and three-particle systems of identical scalars, it remains only to extend item 3 to any number of $\mathcal{K}_{2}$ poles in any angular-momentum channels, and then to combine items 2 and 3 to describe $\mathbf{2} \rightarrow \mathbf{3}$ systems with resonant subprocesses. Beyond this, the remaining extensions to general two- and three-particle systems require incorporating nonidentical and nondegenerate particles, multiple two- and three-particle channels and, finally, particles with spin. Based on the structure of the results derived so far and on our experience with twoparticle quantization conditions, we expect that all of these extensions will be significantly easier than the derivation presented here.

The approach detailed in this article requires treating the pole in $\mathcal{K}_{2}$ as a pseudoparticle and constructing an effective two-particle state, labeled $\tilde{2}$, built from the pole together with the remaining spectator. From this setup we have derived a quantization condition in the usual form of a determinant involving a finite-volume matrix, $\mathcal{F}$, and a divergence-free $\mathrm{K}$ matrix, $\mathcal{K}_{\mathrm{df}}$, both of which carry matrix indices on the $\tilde{2}+3$ effective channel space. The final aspect of the result presented here is the relation between $\mathcal{K}_{\mathrm{df}}$ and the physical scattering amplitude, denoted $\mathcal{M}_{3}$. The latter has the usual degrees of freedom and in particular carries no memory of the unphysical $\tilde{2}$ channel.

One of the central questions raised by this derivation, to be further explored in future work, is whether it is really necessary or natural to explicitly treat the $\mathcal{K}_{2}$ poles as we have done. One motivation for this approach follows from considering, e.g., isospin two $\pi \pi \pi$ scattering for varying quark masses. For physical-mass pions, in the allowed energy range of $3 M_{\pi}<\sqrt{s}<5 M_{\pi}$, the energy of the $\pi \pi$ subsystem is well below the $\rho$ mass and therefore well below any poles in $\mathcal{K}_{2}$. Thus, for this system, the formalism of item 1 above is appropriate. By contrast, for sufficiently heavy pions the $\rho$ is stable so that one requires the formalism of item 2 to describe the $\rho \pi \rightarrow \pi \pi \pi$ scattering amplitude. The latter depends on a two-channel version of $\mathcal{K}_{\mathrm{df}}$ represented by a two-by-two matrix with indices 2 and $3 .{ }^{19}$ Since one can, at least in principle, vary the quark masses continuously between these two scenarios, it is necessary to understand how the quantization conditions transition between the two different matrix spaces.

The result of this work provides a natural answer to this question. As the quark mass increases from the physical point, the $\rho$ pole moves into the sampled energy range and the corresponding pole in $\mathcal{K}_{2}$ is treated by opening an effective $\tilde{2}$ channel. If the quark mass is further increased, this pole location moves closer to the two-particle threshold until it drops below, leading to a stable $\rho$. Note that, even for the case of $M_{\rho}<2 M_{\pi}$, if the mass hierarchy is such that $\kappa^{2} \equiv M_{\pi}^{2}-M_{\rho}^{2} / 4 \ll M_{\pi}^{2}$, i.e., the state is shallow, then the quantization condition derived here should be used to properly incorporate

\footnotetext{
${ }^{19}$ Strictly speaking the only available $\mathbf{2} \rightarrow \mathbf{3}$ formalism requires that all particles in the two- and three-particle states are identical. However based on the nature of the derivation, and the corresponding results in the two-particle sector, it is quite clear that the basic structure of the quantization conditions, in particular the appearance of channel indices, will persist in the case of nonidentical particles.
} 
potentially large volume effects of the form $e^{-\kappa L}$, arising from the large size of the weakly bound state. If the quark masses are instead chosen very large, such that $\kappa>M_{\pi}$, then the finite size of the $\rho$ can be neglected and the two-to-three formalism may be applied.

We further remark that the key difference between the case of the unphysical $\tilde{2}$ and the physical 2 channels is that the off-diagonal elements of $\mathcal{F}$ vanish only in the latter case. Future work is needed to understand exactly how the result derived here can be used to recover to the case of physical $\mathbf{2} \rightarrow \mathbf{3}$ scattering considered previously. Conversely, we recall that the elimination of off-diagonal elements in the $\mathbf{2} \rightarrow \mathbf{3}$ formalism of Ref. [7] required construction of the cutoff function $H(\vec{k})$ such that the finite-volume cuts of one- and two-particle subspaces (within the two- and three-particle states respectively) did not overlap. The results of this work could also allow one to explore more freedom in the definition of $H(\vec{k})$, at the cost of allowing unsuppressed off-diagonal entries in the finite-volume matrix.

Although these observations give some motivation for the $\tilde{2}$ effective channel, it is nonetheless possible that one might reformulate the results without this unphysical aspect. We are motivated to consider this in more detail especially following the demonstration in Sec. IV B that all entries of $\mathcal{F}$ do not contain $\mathcal{K}_{2}$ poles. We note, in addition, that our result requires special treatment of $\mathcal{K}_{2}$ poles regardless of the sign of the residue. Thus also poles with no connection to a resonance state must be separated out. In this case we can provide no physical motivation for this mathematical necessity.

Having removed the largest limitation of our previous formalism, we think it is now feasible to arrive at a quantization condition for completely general two- and three-particle systems. Even after this is achieved, several open issues still remain to be addressed. First, we hope to understand simplifications in both the derivation and the final result that can be made without adding any approximations. We have a sense that these can be identified by better understanding the relation of this work to Refs. $[11,12,14]$, and by studying the pole structures of the final quantities appearing in our results. Second, we plan to understand systematic approximations and truncations. This will likely involve subducing the quantization condition to irreducible representations of the finite-volume symmetry groups and truncating the angular momentum basis as is done in all two-particle studies. Third, we intend to continue our numerical investigations of these results, along the lines of Ref. [16]. Fourth, and finally, we aim to implement this formalism in numerical LQCD calculations. To do so, it is necessary to identify a set of possible functional forms for the scattering amplitudes. Input from the dispersive and amplitude analysis communities is likely to play a key role in this next step. [See Refs. [34,35] for significant progress on this front.]

\section{ACKNOWLEDGMENTS}

The work of S. R. S. was supported in part by the United States Department of Energy Grant No. DE-SC0011637. R. A. B. acknowledges support from U.S. Department of Energy Contract No. DE-AC05-06OR23177, under which Jefferson Science Associates, LLC, manages and operates Jefferson Lab. The authors thank A. Szczepaniak, A. Pilloni, J. Dudek and the late M. Pennington for useful discussions.

\section{APPENDIX A: FACTORIZATION OF THE OFF-SHELL TWO-PARTICLE K MATRIX AT THE POLE}

The aim of this Appendix is to present a derivation of Eq. (13) and its consequences. We first consider K matrix poles above threshold and turn at the end to the case of subthreshold poles.

We begin by reviewing the constraints that unitarity places on two-particle scattering amplitudes. The $\mathrm{S}$ matrix is related to the on-shell scattering amplitude in the standard way,

$$
S_{2}^{(\ell)}=1+2 i \tilde{\rho}_{2} \mathcal{M}_{2 ; \text { on;on }}^{(\ell)}
$$

where we have introduced $\tilde{\rho}_{2}=i \tilde{\rho}$ with $\tilde{\rho}$ defined in Eq. (10). For the purpose of this Appendix, $\tilde{\rho}_{2}$ is more convenient, in particular because it is real above threshold. On the physical scattering axis, i.e., for real energies above threshold on the physical sheet, the $\mathrm{S}$ matrix is unitary, implying

$$
\operatorname{Im}\left(\mathcal{M}_{2 ; \text { on;on }}^{(\ell)}\right)=\mathcal{M}_{2 ; \text { on;on }}^{(\ell)} \tilde{\rho}_{2} \mathcal{M}_{2 ; \text { on;on }}^{(\ell \dagger} .
$$

Given that $\tilde{\rho}_{2}$ is finite, this result prohibits $\mathcal{M}_{2 ; \text { on;on }}^{(\ell)}$ from having poles on the physical axis (since the left-hand side would then have a single pole and the right-hand side a double pole). Of course, poles below threshold for real $s$, corresponding to bound states, are allowed, since this constraint applies only for real energies above threshold.

Unitarity alone cannot put constraints on the analytic structure of off-shell scattering amplitudes. Instead, as described in the main text, we consider this system as a generic effective field theory, with quantities calculated to all orders in perturbation theory. In this context we can connect the off- and on-shell scattering amplitudes, as we now show.

The ingredients we need are, first, the product of two fully dressed propagators with the appropriate symmetry factor,

$$
\Delta_{2} \equiv \frac{1}{2} \Delta(P-k) \Delta(k),
$$

and, second, the fully off-shell Bethe-Salpeter kernel $B_{2 ; \text { off;off }}^{(\ell)}$. We recall that the latter is defined as the sum of all amputated two-to-two diagrams that are two-particle irreducible in the $\mathrm{s}$ channel. ${ }^{20}$ The pair of subscripts

\footnotetext{
${ }^{20}$ As noted in the main text, we are implicitly making a choice of single-particle interpolating operator when defining this kernel. None of the subsequent considerations depend on this choice.
} 
indicates that both initial and final states are off shell. The on-shell versions have the same definitions except that the 4-momenta in either one or both states are set to the physical values, $p^{2} \rightarrow m^{2}$. In terms of these building blocks, the off-shell amplitude can be written as an iteration of s-channel two-particle loops,

$$
\begin{aligned}
i \mathcal{M}_{2 ; \mathrm{off} ; \mathrm{off}}^{(\ell)}= & i B_{2 ; \mathrm{off} ; \mathrm{off}}^{(\ell)}+\int i B_{2 ; \mathrm{off} ; \mathrm{off}}^{(\ell)} \Delta_{2} i B_{2 ; \text { off;off }}^{(\ell)} \\
& +\iint i B_{2 ; \text { off;off }}^{(\ell)} \Delta_{2} i B_{2 ; \mathrm{off} ; \mathrm{off}}^{(\ell)} \Delta_{2} i B_{2 ; \mathrm{off} ; \mathrm{off}}^{(\ell)}+\cdots,
\end{aligned}
$$

where the integrals are over the loop momenta, e.g., over $k$ in Eq. (A3).

Fully or partially on-shell amplitudes are then given by appropriate changes to the subscripts, e.g.,

$$
\begin{aligned}
i \mathcal{M}_{2 ; \mathrm{on} ; \mathrm{on}}^{(\ell)}= & i B_{2 ; \mathrm{on} ; \mathrm{on}}^{(\ell)}+\int i B_{2 ; \mathrm{on} ; \mathrm{off}}^{(\ell)} \Delta_{2} i B_{2 ; \mathrm{off} \text { on }}^{(\ell)} \\
& +\iint i B_{2 ; \mathrm{on} ; \mathrm{off}}^{(\ell)} \Delta_{2} i B_{2 ; \mathrm{off} ; \mathrm{off}}^{(\ell)} \Delta_{2} i B_{2 ; \mathrm{off} ; \mathrm{on}}^{(\ell)}+\cdots
\end{aligned}
$$

These results can be used to rewrite the on-shell amplitude in three useful forms,

$$
\begin{aligned}
i \mathcal{M}_{2 ; \text { on;on }}^{(\ell)}= & i B_{2 ; \text { on;on }}^{(\ell)}+\int i B_{2 ; \text { on;off }}^{(\ell)} \Delta_{2} i \mathcal{M}_{2 ; \text { off;on }}^{(\ell)} \\
= & i B_{2 ; \text { on;on }}^{(\ell)}+\int i \mathcal{M}_{2 ; \text { on;off }}^{(\ell)} \Delta_{2} i B_{2 ; \text { off;on }}^{(\ell)} \\
= & i B_{2 ; \text { on;on }}^{(\ell)}+\int i B_{2 ; \text { on;off }}^{(\ell)} \Delta_{2} i B_{2 ; \text { off;on }}^{(\ell)} \\
& +\iint i B_{2 ; \text { on;off }}^{(\ell)} \Delta_{2} i \mathcal{M}_{2 ; \text { off;off }}^{(\ell)} \Delta_{2} i B_{2 ; \text { off;on }}^{(\ell)} .
\end{aligned}
$$

To proceed, we assume that resonances in $\mathcal{M}_{2 ; \text { on;on }}^{(\ell)}$ arise by the iteration of the two-particle loops in Eq. (A5) and are not present in the kernel $B_{2}^{(\ell)}$ itself (whether on or off shell). In other words, since $B_{2}^{(\ell)}$ has no intermediate states that are on shell in the kinematic range of interest, $4 m^{2}<s<$ $16 m^{2}$ (or $4 m^{2}<s<9 m^{2}$ if there is no G-paritylike symmetry), it can be treated as a nearly local two-particle interaction, and it is the iteration of this interaction that leads to resonances. Given this assumption, $B_{2}^{(\ell)}$ has no s-channel singularities on the physical axis. This is a key input in the following arguments. We note that $B^{(\ell)}$ can have t- and u-channel singularities (e.g., the left-hand cut) but these occur for $s \leq 0$ and are thus outside of the kinematic range of interest.

Given the inputs that neither $\mathcal{M}_{2 ; \text { on;on }}^{(\ell)}$ nor $B_{2}^{(\ell)}$ have poles on the physical axis, it follow from Eqs. (A6), (A7) and (A8), respectively, that $\mathcal{M}_{2 ; \text { off;on }}^{(\ell)}, \mathcal{M}_{2 ; \text { on;off }}^{(\ell)}$ and $\mathcal{M}_{2 \text {;off;off }}^{(\ell)}$ cannot have such poles either. Of course, all these quantities can have poles in the complex plane corresponding to resonances, but the key point here is that the off-shell amplitudes inherit from $\mathcal{M}_{2 \text {;on;on }}^{(\ell)}$ the absence of poles on the real axis above threshold.

With this in hand, we can finally turn our attention to the $\mathrm{K}$ matrix. Again, unitarity alone places no constraints on the K matrix, but we can use its all-orders effective field theory definition to relate it to the scattering amplitude. Indeed, whether on or off shell, the two quantities differ only by the replacement of the $i \epsilon$ prescription in twoparticle loops with the principal-value prescription. The difference in these definitions is proportional to $\tilde{\rho}_{2}$ and a $\delta$-function that places the states on shell. From this we find that the fully off-shell $\mathrm{K}$ matrix can be written as [see also Eq. (9)]

$$
\begin{aligned}
i \mathcal{K}_{2 ; \text { off;off }}^{(\ell)} & =i \mathcal{M}_{2 ; \text { off;off }}^{(\ell)}-i \mathcal{M}_{2 ; \text { off;on }}^{(\ell)} \tilde{\rho}_{2} i \mathcal{M}_{2 ; \text { on;off }}^{(\ell)}+i \mathcal{M}_{2 ; \text { off;on }}^{(\ell)} \tilde{\rho}_{2} i \mathcal{M}_{2 ; \text { on;on }}^{(\ell)} \tilde{\rho}_{2} i \mathcal{M}_{2 ; \text { on;off }}^{(\ell)}+\cdots \\
& =i \mathcal{M}_{2 ; \text { off;off }}^{(\ell)}-i \mathcal{M}_{2 ; \text { off;on }}^{(\ell)} \tilde{\rho}_{2}\left[\frac{1}{1+i \mathcal{M}_{2 ; \text { on;on }}^{(\ell)} \tilde{\rho}_{2}}\right] i \mathcal{M}_{2 ; \text { on;off }}^{(\ell)} \\
& =i \mathcal{M}_{2 ; \text { off;off }}^{(\ell)}-i \mathcal{M}_{2 ; \text { off;on }}^{(\ell)} \tilde{\rho}_{2}\left[\frac{1}{\mathcal{M}_{2 ; \text { on;on }}^{(\ell)-1}+i \tilde{\rho}_{2}}\right] \mathcal{M}_{2 ; \text { on;on }}^{(\ell)-1} i \mathcal{M}_{2 ; \text { on;off }}^{(\ell)} \\
& =i \mathcal{M}_{2 ; \text { off;off }}^{(\ell)}-i \mathcal{M}_{2 ; \text { off;on }}^{(\ell)} \tilde{\rho}_{2} \mathcal{K}_{2 ; \text { on;on }}^{(\ell)} \mathcal{M}_{2 ; \text { on;on }}^{(\ell)-1} i \mathcal{M}_{2 ; \text { on;off }}^{(\ell)} .
\end{aligned}
$$

In the last step, we have expressed the off-shell $\mathcal{K}_{2}$ in terms of its on-shell form. This gives the desired result, Eq. (13), when working above threshold so that $H=1$. The key point is that, on the right-hand side of Eq. (A9), the only quantity that has poles on the physical axis is $\mathcal{K}_{2 \text {;on;on }}^{(\ell)}$. Thus we conclude that $\mathcal{K}_{2 \text {;off;off }}^{(\ell)}$ must share these poles with $\mathcal{K}_{2 \text {;on;on }}^{(\ell)}$ in order for the equality to hold.

The second result we wish to show is the factorization of the residues of poles in $\mathcal{K}_{2 ; \text { off;off }}^{(\ell)}$. To do this we note that the on-shell scattering amplitude is purely imaginary at the poles of $\mathcal{K}_{2 \text {;on;on }}^{(\ell)}$, 


$$
\mathcal{M}_{2 ; \text { on;on }}^{(\ell)-1} \rightarrow-i \tilde{\rho}_{2} \text {. }
$$

Therefore, near the poles, the off- and on-shell $\mathrm{K}$ matrices are related by

$$
i \mathcal{K}_{2 ; \text { off;off }}^{(\ell)} \sim Z_{2 ; \text { off;on }}^{(\ell)} i \mathcal{K}_{2 ; \text { on;on }}^{(\ell)} Z_{2 ; \text { on;off }}^{(\ell)},
$$

where $Z_{2 ; \text { off;on }}^{(\ell)}=-i \mathcal{M}_{2 \text {;off;on }}^{(\ell)} \tilde{\rho}_{2}$ and $Z_{2 ; \text { on;off }}^{(\ell)}=-i \tilde{\rho}_{2} \mathcal{M}_{2 ; \text { on;off }}^{(\ell)}$. These two quantities depend, respectively, only on the final (initial) momenta, thus demonstrating the claimed factorization of momentum dependence. Both quantities equal unity when the corresponding external legs are placed on shell. Comparing the definitions of the residues of poles in on- and off-shell K matrices, given in Eqs. (16) and (15), respectively, we see that

$$
\Gamma_{J}\left(M^{2}, a^{\prime 2}, b^{\prime 2}\right)\left(a_{2, k}^{\prime *}\right)^{J}=\Gamma_{J}\left(q_{2, k}^{*}\right)^{J} Z_{2 ; \text { on;off }}^{(J)},
$$

with a similar relation for $Z_{2 ; \text { off;on }}^{(J)}$.

Before concluding this Appendix, we return to the situation in which the $\mathrm{K}$ matrix has poles for real values of the energy lying below threshold. In this case, Eq. (A9) continues to hold-since it is based on a diagrammatic analysis-except that $\tilde{\rho}_{2}$ becomes $i \tilde{\rho} H$, with the factor of $H$ required by our definition of $\mathcal{K}_{2}$ [see Eq. (9)]. Thus we obtain Eq. (13) also when working below threshold, and consequently it remains true that poles in the onshell $\mathrm{K}$ matrix appear in its off-shell extension, in the same locations.

There is, however, an additional issue that must be considered. This arises because the scattering amplitude itself can have poles for real, subthreshold energies, corresponding to bound states. At such poles, the on-shell $\mathrm{K}$ matrix becomes $\mathcal{K}_{2 \text {,on;on }}^{(\ell)} \rightarrow-1 /(\tilde{\rho} H)$, which is real and finite. The issue is whether the off-shell $\mathrm{K}$ matrix is also finite. To see that this is in fact the case, we make use of the factorization of $\mathcal{M}_{2}$ at the pole, allowing us to write

$$
\begin{aligned}
i \mathcal{M}_{2 ; \text { on;on }}^{(\ell)} & \sim \frac{i\left(i g_{\text {on }}\right)^{2}}{\left(s-E_{b}^{2}\right)}, \\
i \mathcal{M}_{2 ; \text { off;on }}^{(\ell)} & \sim \frac{i\left(i g_{\text {off }}\right)\left(i g_{\text {on }}\right)}{\left(s-E_{b}^{2}\right)}, \quad \text { and } \\
i \mathcal{M}_{2 ; \text { off;off }}^{(\ell)} & \sim \frac{i\left(i g_{\text {off }}\right)^{2}}{\left(s-E_{b}^{2}\right)},
\end{aligned}
$$

where $E_{b}$ is the energy of the bound state pole and $s=P_{2, k}^{2}$ is the two-particle c.m. energy. Substituting these results into Eq. (A9) and using the value of $\mathcal{K}_{2 ; \text { on;on }}^{(\ell)}$ at the pole, we find that $\mathcal{K}_{2 \text {;off;off }}^{(\ell)}$ is indeed finite at $s=E_{b}^{2}$.

\section{APPENDIX B: DETAILS OF THE DERIVATION OF RESULTS PRESENTED IN SEC. III}

In this Appendix we present technical details of the derivations outlined in Sec. III.

\section{Derivation of the recursion formula for $C_{L, 0 F}^{(m, n)}$ [Eq. (56)]}

Here we derive Eq. (56) and, in doing so, give complete definitions of the quantities defined therein.

$C_{L, 0 F}^{(m, n)}$ is shown diagrammatically in Fig. 1(b). Here we focus on the next momentum to be converted from a sum to an integral, labeled $q_{m+1}$ in Fig. 1(b). Thus it is convenient to absorb the integrated loops to the left of $q_{m+1}$ into a new end cap $i \mathcal{B}_{\infty}^{(m)}$, and similarly to absorb the summed loops to the right into $i \mathcal{B}_{L}^{(n-m-2)}$, since these new end caps maintain their forms throughout the derivation. This new notation is shown in Fig. 6(a), with the diagrammatic definitions of the end caps $\mathcal{B}_{\infty}^{(m)}$ and $\mathcal{B}_{L}^{(m)}$ sketched, respectively, in
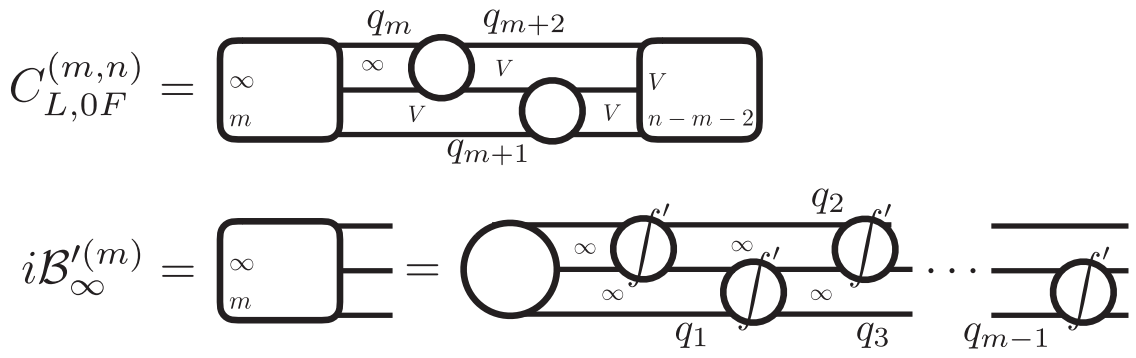

(c)

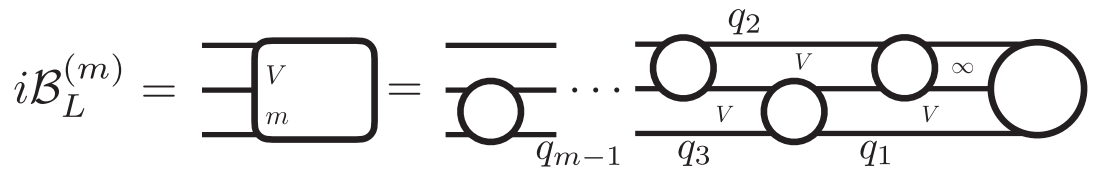

FIG. 6. Diagrammatic definitions for the objects appearing in the initial decomposition of $C_{L, 0 F}^{(m, n)}$, Eq. (B1). The square boxes with rounded corners represent the end caps $i \mathcal{B}$, with the entries inside the box corresponding to the superscripts and subscripts. The infinite-volume and finite-volume versions of these end caps are shown, respectively, in (b) and (c). Remaining notation is as in Fig. 1. 
(a)

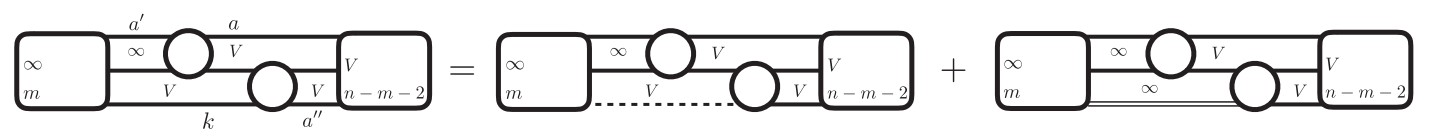

(b)
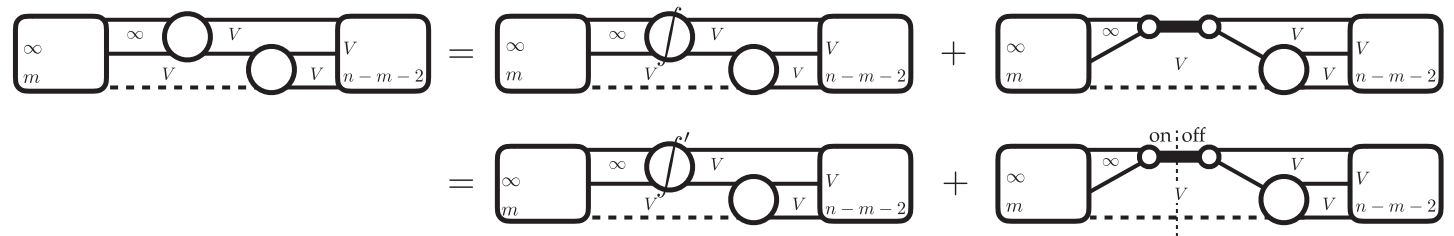

(c)

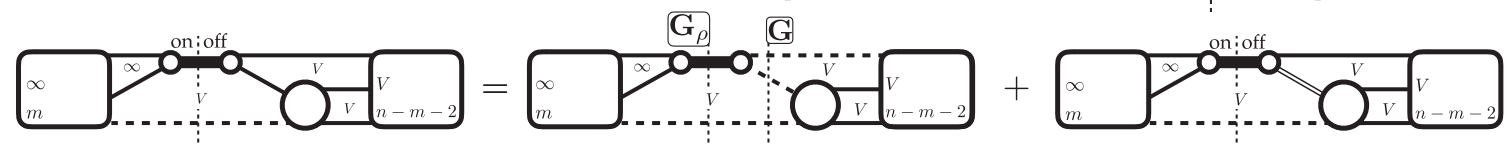

(d)
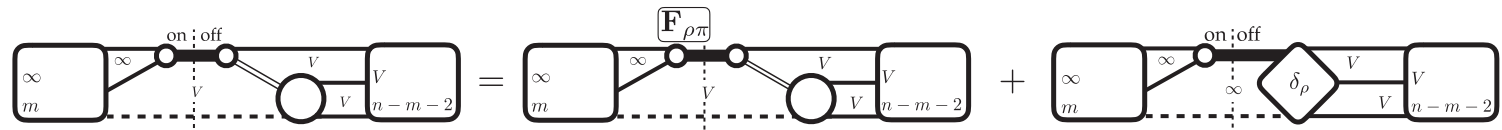

F

(e)
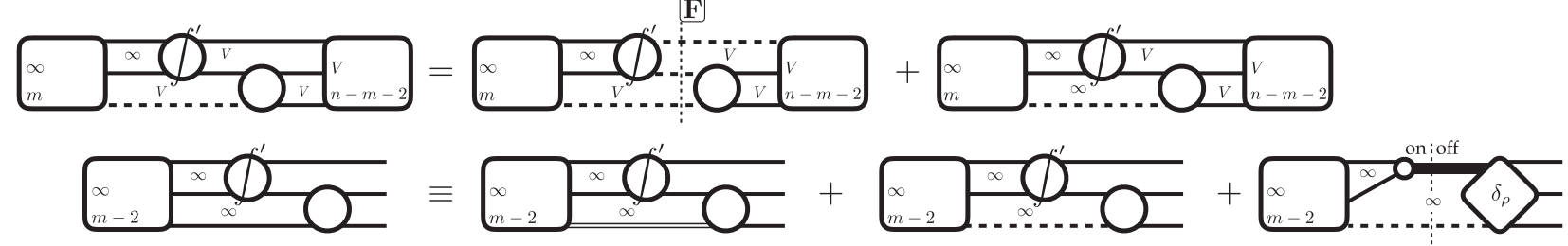

FIG. 7. Summary of the various steps used to derive Eq. (56), the recursion relation for decomposing $C_{L, 0 F}^{(m, n)}$. (a) First the leftmost spectator is split into its pole contribution and a second term that is smooth at the pole and thus contributes to $C_{L, 0 F}^{(m+1, n)}$. (b) Next, in the first term from (a) the leftmost $\mathrm{K}$ matrix is decomposed into a smooth and singular part and the latter is projected partially on shell, as explained in the text. (c) The complete on-shell projection at the K-matrix pole is performed simultaneously with the projection of the exchange propagator, leading to factors of $\mathbf{G}_{\rho}$ and $\mathbf{G}$. (d) This leads to a remainder term that is smooth everywhere except at the K-matrix pole and can be separated into an on-shell term and another contribution to $C_{L, 0 F}^{(m+1, n)}$. (e) The remaining piece to decompose, in which the rightmost $\mathrm{K}$ matrix is smooth, leads to an $F$ cut in the middle of the diagram and yet another contribution to $C_{L, 0 F}^{(m+1, n)}$. (f) Finally we summarize all terms entering $\mathcal{B}_{\infty}^{(m+1)}$, collected from (a), (d) and (e) above. The notation is as in Figs. 1 and 6, with the addition that dashed lines represent on-shell propagators. See text for further discussion.

Figs. 6(b) and 6(c). ${ }^{21}$ The superscripts on the $\mathcal{B}$ s denote the number of factors of $i \mathcal{K}_{2}$ or its smooth counterpart that they contain. Note that these end caps are closely related to $A_{3}^{\prime(m, u)}$ and $A_{L, 3}^{(m, u)}$, respectively, quantities discussed in the main text. The differences are that the $\mathcal{B}$ end caps are not projected on shell, and also not decomposed into spherical harmonics. The $\mathcal{B}$ end caps are shown in the figures as open squares with rounded corners.

Using this notation, we can explicitly display the relevant part of $C_{L, 0 F}^{(m, n)}$,

$$
\begin{aligned}
C_{L, 0 F}^{(m, n)}= & \frac{1}{4} \sum_{k, a, a^{\prime \prime}} \int_{a^{\prime}} i \mathcal{B}_{\infty}^{(m)}\left(k, a^{\prime}\right) \Delta\left(a^{\prime}\right) \Delta\left(b^{\prime}\right) i \mathcal{K}_{2, \mathrm{off}}\left(a^{\prime}, b^{\prime},-a\right) \\
& \times \Delta(k) \Delta(a) \Delta(b) i \mathcal{K}_{2, \mathrm{off}}\left(k, b,-a^{\prime \prime}\right) \Delta\left(a^{\prime \prime}\right) \\
& \times \Delta\left(b^{\prime \prime}\right) i \mathcal{B}_{L}^{(n-m-2)}\left(a, a^{\prime \prime}\right) .
\end{aligned}
$$

\footnotetext{
${ }^{21}$ The definition of $\mathcal{B}_{\infty}^{(m)}$ is imprecise, since additional terms are included for each extra factor of $i \mathcal{K}_{2}$ that is added. This is explained in Fig. 7 and the accompanying text.
}

Here we have changed the labels to the more manageable choices shown in Fig. 7(a), and used the definitions $b^{\prime} \equiv P-k-a^{\prime}, b \equiv P-a-k, b^{\prime \prime} \equiv P-a-a^{\prime \prime}$ and

$$
\int_{a} \equiv \widetilde{\mathrm{PV}} \int \frac{d^{4} a}{(2 \pi)^{4}}, \quad \sum_{k} \equiv \int \frac{d k^{0}}{2 \pi} \frac{1}{L^{3}} \sum_{\vec{k}}
$$

The quantity $\mathcal{K}_{2, \text { off }}$ is the fully off-shell two-particle $\mathrm{K}$ matrix defined as in Ref. [5], with the first two labels denoting the outgoing momenta, and the third one of the incoming momenta. Note that in Appendix A we refer to this $\mathrm{K}$ matrix as $\mathcal{K}_{2 \text {;off,off }}$; here the double subscript is not necessary. The expression (B1) holds for $n-m>2$ and $n>2$, which is the case shown in Fig. 7(a). For $n-m=2$ and $n \geq 2$ the sum over $a^{\prime \prime}$ is replaced by an integral. Other cases are simpler and are discussed at the end.

To derive Eq. (56), we begin by making the substitution

$$
\Delta(k)=(2 \pi) \delta\left(k^{0}-\omega_{k}\right) \frac{1}{2 \omega_{k}}+\mathcal{R}(k)
$$


thereby separating the particle-pole contribution to the propagator (which is the only part that can lead to singularities as a function of $k$ ) from the remainder, $\mathcal{R}(k)$. This is shown in Fig. 7(a) where, just as in Fig. 3, the pole is shown by a dashed line and the remainder by double solid lines. In the contribution of $\mathcal{R}(k)$ to $C_{L, 0 F}^{(m, n)}$, we can replace the sum over $k$ with an integral, leading to a contribution to $C_{L, 0 F}^{(m+1, n)}$, shown as the second term on the right-hand side of Fig. 7(a). Thus we focus only on the particle-pole contribution, the first term on the right side of $7(\mathrm{a})$, in the following.

The next step is to insert a variant of Eq. (15) for the lefthand $\mathrm{K}$ matrix

$i \mathcal{K}_{2, \mathrm{off}}\left(a^{\prime}, b^{\prime},-a\right)=4 \pi Y_{J M_{J}}^{*}\left(\hat{a}_{2, k}^{\prime *}\right)\left(a_{2, k}^{\prime *}\right)^{J} i \Gamma_{J}\left(M^{2}, a^{\prime 2}, b^{\prime 2}\right) \frac{i \eta_{J} H_{\rho}(\vec{k})}{\left(P_{2, k}^{2}-M^{2}\right)} i \Gamma_{J}\left(M^{2}, a^{2}, b^{2}\right)\left(a_{2, k}^{*}\right)^{J} Y_{J M_{J}}\left(\hat{a}_{2, k}^{*}\right)+i \tilde{\mathcal{K}}_{2, \mathrm{off}}\left(a^{\prime}, b^{\prime},-a\right)$.

Here we have added back in the spherical harmonics needed to recreate the full $\mathrm{K}$ matrix. Note that, by assumption, the pole appears only in the $J$ th partial wave, while the second, smooth term includes contributions from all partial waves. In the first term of Eq. (B4), $J$ is fixed, while $M_{J}$ is summed from $-J$ to $J$. Note also, as compared to Eq. (15), we have included the UV regulator $H_{\rho}$ in the pole term. This can be added since, by construction, $1-H_{\rho}$ cancels the K-matrix pole, and thus leads to a smooth contribution that can be absorbed into $\tilde{\mathcal{K}}_{2 \text {,off }}$.

The result of this insertion is shown in Fig. 7(b). We first consider the K-matrix pole contribution, which is represented by the second term on the right-hand side of Fig. 7(b), and has the explicit expression

$$
\begin{aligned}
C_{L, 0 F}^{(m, n)} \supset & \frac{1}{2} \frac{1}{L^{3}} \sum_{\vec{k}} \sum_{a, a^{\prime \prime}} \int_{a^{\prime}} i A_{2 ; M_{J}}^{\prime(m)}(\vec{k}) \frac{i \eta_{J} H_{\rho}(\vec{k})}{\left(P_{2, k}^{2}-M^{2}\right)} i \Gamma_{J}\left(M^{2}, a^{2}, b^{2}\right)\left(a_{2, k}^{*}\right)^{J} \frac{1}{2 \omega_{k}} \sqrt{4 \pi} Y_{J M_{J}}\left(\hat{a}_{2, k}^{*}\right) \\
& \times \Delta(a) \Delta(b) i \mathcal{K}_{2, \text { off }}\left(k, b,-a^{\prime \prime}\right) \Delta\left(a^{\prime \prime}\right) \Delta\left(b^{\prime \prime}\right) i \mathcal{B}_{L}^{(n-1)}\left(a, a^{\prime \prime}\right),
\end{aligned}
$$

where

$$
\begin{aligned}
i A_{\tilde{2} ; M_{J}}^{\prime(m)}(\vec{k}) \equiv & \frac{1}{2} \int_{a^{\prime}} i B_{\infty}^{(m-1)}\left(k, a^{\prime}\right) \Delta\left(a^{\prime}\right) \\
& \times \Delta\left(b^{\prime}\right) \sqrt{4 \pi} Y_{J M_{J}}^{*}\left(\hat{a}_{2, k}^{\prime *}\right)\left(a_{2, k}^{\prime *}\right)^{J} i \Gamma_{J}\left(M^{2}, a^{\prime 2}, b^{\prime 2}\right) .
\end{aligned}
$$

In both of these equations $k$ is on shell, $k^{\mu}=\left(\omega_{k}, \vec{k}\right)$. In the figures, we represent the factors of $i \Gamma_{J}$ by small closed circles, and the K-matrix pole by a thick horizontal line.

At this stage $A_{\tilde{2} ; M_{J}}^{\prime(m)}(\vec{k})$ is not evaluated at the $\mathrm{K}$ pole, i.e., $P_{k}^{2} \neq M^{2}$. We can pick out the on-shell part (where on shell here refers to the $\tilde{2}$ state consisting of a particle plus the K-matrix pole) by hand, by introducing a $\delta$ operator analogous to those used in Ref. [5],

$$
A_{\tilde{2} ; M_{J}}^{\prime(m)}(\vec{k}) \equiv A_{\tilde{2} ; M_{J} \ell^{\prime} m^{\prime}}^{\prime(m)} \mathcal{Y}_{2 ; \ell^{\prime} m^{\prime}}\left(\vec{k}^{*}\right)+\delta_{\rho} A_{\tilde{2} ; M_{J}}^{\prime(m)}(\vec{k}),
$$

where the on-shell value of $A_{\tilde{2}}^{\prime}$ is

$$
A_{\tilde{2} ; M_{J} \ell^{\prime} m^{\prime}}^{\prime(m)} \sqrt{4 \pi} Y_{\ell^{\prime} m^{\prime}}\left(\hat{k}^{*}\right) \equiv A_{\tilde{2} ; M_{J}}^{\prime(m)}\left(q_{\rho}^{*} \hat{k}^{*}\right) .
$$

Here we are using the definitions of $\mathcal{Y}_{2}$ from Eq. (34) and of $q_{\rho}^{*}$ from Eq. (6). This step is represented by the second line in Fig. 7(b), where in the second term we use the label "on" to indicate those quantities for which the $\rho \pi$ relative momentum has been set to its on-shell value, $\vec{k}^{*} \rightarrow q_{\rho}^{*} \hat{k}^{*}$. If $\vec{k}$ is left at its original value, then we use the label "off." The $\delta_{\rho}$ operator cancels the K-matrix pole, and thus its contribution can be absorbed into that from $\tilde{\mathcal{K}}_{2}$ to $C_{L, 0 F}^{(m, n)}$. This is indicated by the prime on the integrated $\mathrm{K}$-matrix symbol in the first term on the right-hand side of Fig. 7(b). We return to this contribution later. Equation (B8) completes the definition of the infinite-volume end cap $A_{\tilde{2} ; M_{J} \ell m}^{\prime(m)}$.

Substituting the on-shell term from Eq. (B7) into Eq. (B5), we obtain the second term on the second line of Fig. 7(b). The explicit expression is 


$$
\begin{aligned}
C_{L, 0 F}^{(m, n)} \supset & \frac{1}{2} \sum_{\vec{k}} \sum_{a, a^{\prime \prime}} \int_{a^{\prime}} i A_{\tilde{2} ; M_{J} \ell^{\prime} m^{\prime}}^{\prime(m)} \mathcal{Y}_{\tilde{2}, \ell^{\prime} m^{\prime}}\left(\vec{k}^{*}\right) \frac{i \eta_{J} H_{\rho}(\vec{k})}{\left(P_{2, k}^{2}-M^{2}\right)} i \Gamma_{J}\left(M^{2}, a^{2}, b^{2}\right)\left(a_{2, k}^{*}\right)^{J} \frac{1}{2 \omega_{k} L^{3}} \sqrt{4 \pi} Y_{J M_{J}}\left(\hat{a}_{2, k}^{*}\right) \\
& \times \Delta(a) \Delta(b) i \mathcal{K}_{2, \text { off }}\left(k, b,-a^{\prime \prime}\right) \Delta\left(a^{\prime \prime}\right) \Delta\left(b^{\prime \prime}\right) i \mathcal{B}_{L}^{(n-1)}\left(a, a^{\prime \prime}\right) .
\end{aligned}
$$

The final step for this term is to introduce a " $G$ cut" through the $a, b$ and $k$ propagators, following the approach of Ref. [5]. This cut places all three particles on shell, but in a different manner to the left and the right of the cut. In both cases, the spectator momentum is unchanged $(\vec{k}$ to the left, and $\vec{a}$ to the right), while the interacting pair has its momenta rescaled in their c.m. frame. The $G$-cut term thus replaces $\Gamma_{J}\left(M^{2}, a^{2}, b^{2}\right)$ with the fully on shell $\Gamma_{J}$, and $a_{2, k}^{*}$ with $q_{2, k}^{*}$. This is shown in Fig. 7(c). Using the definitions given in Sec. II, we find that the $G$-cut contribution [the first term on the right-hand side of Fig. 7(c)] is

$$
\begin{aligned}
C_{L, 0 F}^{(m, n)} \supset & i A_{\tilde{2} ; M_{\ell} \ell^{\prime} m^{\prime}}^{(m)} i G_{\rho ; M_{J} \ell^{\prime} m^{\prime} ; k \ell^{\prime \prime} m^{\prime \prime}} i \Gamma_{J} \\
& \times \frac{1}{2 \omega_{k} L^{3}} i G_{k \ell^{\prime \prime} m^{\prime \prime} ; a \ell^{\prime \prime \prime} m^{\prime \prime \prime}} i A_{L, 3 ; a \ell^{\prime \prime \prime} m^{\prime \prime \prime}}^{(n-m, u)} \\
= & \mathbf{A}_{\tilde{2}}^{\prime(m)} \mathbf{G}_{\rho} \boldsymbol{\Gamma} \mathbf{G} \mathbf{A}_{L, 3}^{(n-m, u)}
\end{aligned}
$$

where all repeated indices are summed in the middle quality and left implicit in the last. This is the third term on the righthand side of the first line of Eq. (56).

The cut we have just discussed is the most singular that arises, having the $\mathcal{K}_{2}$ pole $\left(\mathbf{G}_{\rho}\right)$ and the three-particle pole (G) separated only by the constant $\boldsymbol{\Gamma}$. It is possible for both poles to go on shell simultaneously, for special values of $\vec{k}$ and $\vec{a}$. We stress that these potential double poles appear only in sums over the spectator momenta, and not in sumintegral differences. Thus we do not need to introduce a generalized zeta function to describe them, unlike, for example, in the analysis of finite-volume effects in twoparticle matrix elements [36].

The difference between Eqs. (B9) and (B10), represented by the last term of Fig. 7(c), has no three-particle singularity, but still retains the K-matrix pole. The absence of this singularity is shown in Fig. 7(c) by the double line for the $b$ propagator in the last term. We now project the quantity to the right of this pole on shell using the $\delta_{\rho}$ operator introduced above in Eq. (B7), but now acting to the right. This is shown in Fig. 7(d), leading to the final term on the first line of Eq. (56),

$$
\mathbf{A}_{2}^{\prime(m+1)} \mathbf{F}_{\rho \pi} \mathbf{A}_{L, \tilde{2}}^{(n-m)},
$$

in which $\mathbf{F}_{\rho \pi}$ acts like a cut, and provides an implicit definition of $A_{L, \tilde{2}}$. The term involving $\delta_{\rho}$ removes the
$\mathrm{K}$-matrix pole, and is thus free of singularities. For this term the sum over $k$ can be replaced by an integral, providing an additional contribution to $C_{L, 0 F}^{(m+1, n)}$.

Finally we consider the part involving the smooth part of the left-hand $\mathcal{K}_{2}$, i.e., the first term in the second line of Fig. 7(b), whose explicit expression is

$$
\begin{aligned}
C_{L, 0 F}^{(m, n)} \supset & \frac{1}{4} \frac{1}{L^{3}} \sum_{\vec{k}} \sum_{a, a^{\prime \prime}} \int_{a^{\prime}} i B_{\infty}^{(m)}\left(k, a^{\prime}\right) \Delta\left(a^{\prime}\right) \\
& \times \Delta\left(b^{\prime}\right) i \tilde{\mathcal{K}}_{2, \mathrm{off}}^{\prime}\left(a^{\prime}, b^{\prime},-a\right) \frac{1}{2 \omega_{k}} \\
& \times \Delta(a) \Delta(b) i \mathcal{K}_{2, \mathrm{off}}\left(k, b,-a^{\prime \prime}\right) \Delta\left(a^{\prime \prime}\right) \\
& \times \Delta\left(b^{\prime \prime}\right) i \mathcal{B}_{L}^{(n-m-2)}\left(a, a^{\prime \prime}\right) .
\end{aligned}
$$

As noted above, another term with the same pole structure has been implicitly absorbed into this expression. We represent this by adding a prime to $\tilde{\mathcal{K}}_{2, \text { off }}^{\prime}$. The situation is now just as in Ref. [5], since the K-matrix pole is absent. Thus we can replace the sum over $\vec{k}$ with an integral plus the difference, the latter giving rise to an $F$ cut. We do not present the details as they have been presented in Ref. [5]. This step is shown in Fig. 7(e). The $F$ cut gives the second term on the right-hand side of the first line of Eq. (56), which has the form

$$
2 \mathbf{A}_{3}^{\prime(m+1, s)} \mathbf{F} \mathbf{A}_{L, 3}^{(n-m-1, u)},
$$

while the integral leads to the final contribution to $C_{L, 0 F}^{(m+1, n)}$.

As we have progressed through this derivation, we have picked up three contributions that can be absorbed into $C_{L, 0 F}^{(m+1, n)}$. In fact, given our definition $C_{L, 0 F}$ in terms of the $\mathcal{B}$ end caps, Eq. (B1), the contributions are specifically absorbed into $\mathcal{B}_{\infty}^{(m+1)}$. This is shown in Fig. 7(f). In this way $\mathcal{B}_{\infty}$ and the meaning of the smooth $\mathcal{K}_{2}$ symbol, $\tilde{\mathcal{K}}_{2}^{\prime}$, are defined recursively, and this feeds into the definitions of the other infinite-volume end caps.

The above discussion holds for $n-m \geq 2$, so that the two $\mathcal{K}_{2}$ factors can be pulled out and dealt with explicitly. The case $n-m=1$ is special, since there is only a single summed loop and the only singularity arises from the pole in $\mathcal{K}_{2}$. The analysis is simpler for this case and leads to the second line in Eq. (56). 


\section{Details on decomposition of $\mathbf{K}_{L}^{(u)}$ described in Sec. III E}

In this Appendix we provide various details in the derivation of Eq. (65) described in Sec. III E. As in the main text, many of these results have been checked using a Mathematica notebook together with the package The NCAlgebra Suite [33]. Equations verified in this way are preceded by the indicator $(\checkmark N C A \checkmark)$.

We begin by solving Eq. (71). By isolating $\mathbf{K}_{L, 33}^{(u, u)}$ in the matrix equation one finds

$$
\mathbf{K}_{L, 33}^{(u, u)}=\frac{1}{1-\mathbf{K}_{L, 33} \mathbf{G}-\mathbf{K}_{2} \mathbf{G}}\left[\mathbf{K}_{2} \mathbf{G} \mathbf{K}_{2}+\mathbf{K}_{L, 33}\left(1+\mathbf{G K}_{2}\right)\right],
$$

which can be rearranged into a compact, symmetric expression $(\checkmark N C A \checkmark)$,

$$
\mathbf{K}_{L, 33}^{(u, u)}=\mathbf{K}_{L, 33}^{(0)}+(1+\mathbf{T G}) \mathbf{K}_{L, 33} \frac{1}{1-\mathbf{G}_{\mathbf{K}} \mathbf{K}_{L, 33}}(1+\mathbf{G T}),
$$

where $\mathbf{K}_{L, 33}^{(0)}$ is defined in Eq. (66). This is identically the 33 component of Eq. (77). To see this, we rewrite the latter equation as

$$
\begin{aligned}
\mathbf{K}_{L}^{(u)} & =\left(\begin{array}{cc}
0 & 0 \\
0 & \mathbf{K}_{L, 33}^{(0)}
\end{array}\right)+\mathcal{E}_{L} \mathbf{K}_{L} \frac{1}{1-\mathcal{G}_{\mathbf{K}} \mathbf{K}_{L}} \mathcal{E}_{R}, \quad(\mathrm{~B} 16) \\
& =\left(\begin{array}{cc}
0 & 0 \\
0 & \mathbf{K}_{L, 33}^{(0)}
\end{array}\right)+\left(\begin{array}{cc}
1 & 0 \\
0 & 1+\mathbf{T G}
\end{array}\right)\left(\begin{array}{ll}
\mathbf{K}_{L, \tilde{2} \tilde{2}} & \mathbf{K}_{L, \tilde{2} 3} \\
\mathbf{K}_{L, 3 \tilde{2}} & \mathbf{K}_{L, 33}
\end{array}\right) \\
& \times\left[1-\left(\begin{array}{cc}
0 & 0 \\
0 & \mathbf{G}_{\mathbf{K}}
\end{array}\right)\left(\begin{array}{ll}
\mathbf{K}_{L, \tilde{2} \tilde{2}} & \mathbf{K}_{L, \tilde{2} 3} \\
\mathbf{K}_{L, 3 \tilde{2}} & \mathbf{K}_{L, 33}
\end{array}\right)\right]^{-1}\left(\begin{array}{cc}
1 & 0 \\
0 & 1+\mathbf{G T}
\end{array}\right) .
\end{aligned}
$$

As the two-by-two matrix containing $\mathbf{G}_{\mathbf{K}}$, as well as matrices $\mathcal{E}_{L}$ and $\mathcal{E}_{R}$, project onto the 3 component of their neighbors, it is straightforward to determine the 33 component of this relation and see that it indeed matches Eq. (B15).

We now turn to $\mathbf{K}_{L, \tilde{2} 3}^{(u)}$. Substituting the result for $\mathbf{K}_{L, 33}^{(u, u)}$ into Eq. (73), and simplifying yields $(\boldsymbol{} N C A \checkmark)$

$$
\mathbf{K}_{L, \tilde{2} 3}^{(u)}=\mathbf{K}_{L, \tilde{2} 3} \frac{1}{1-\mathbf{G}_{\mathbf{K}} \mathbf{K}_{L, 33}}(1+\mathbf{G T}) .
$$

The expression for $\mathbf{K}_{L, 3 \tilde{2}}^{(u)}$ can be obtained similarly, with the result being essentially the left-right reflection of Eq. (B18) $(\checkmark N C A \checkmark)$,

$$
\mathbf{K}_{L, 3 \tilde{2}}^{(u)}=(1+\mathbf{T G}) \frac{1}{1-\mathbf{K}_{L, 33} \mathbf{G}_{\mathbf{K}}} \mathbf{K}_{L, 3 \tilde{2}}
$$

Together these results give the $\tilde{2} 3$ and $3 \tilde{2}$ components of Eq. (77) [equivalently Eq. (B17)].

The final quantity we need is $\mathbf{K}_{L, \tilde{2} 2}$. Using the method detailed in the main text for $\mathbf{K}_{L, 33}^{(u, u)}$, we find

$$
\mathbf{K}_{L, \tilde{2} \tilde{2}}=\mathbf{K}_{L, \tilde{2} 3}^{(u)} \mathbf{G K}_{L, 3 \tilde{2}}^{(u)}+\mathbf{K}_{L, \tilde{2} \tilde{2}} .
$$

Substituting (B19) and rearranging leads to $(\boldsymbol{} N C A \checkmark)$

$$
\mathbf{K}_{L, \tilde{2} \tilde{2}}=\mathbf{K}_{L, \tilde{2} \tilde{2}}+\mathbf{K}_{L, \tilde{2} 3} \mathbf{G}_{\mathbf{K}} \frac{1}{1-\mathbf{K}_{L, 33} \mathbf{G}_{\mathbf{K}}} \mathbf{K}_{L, 3 \tilde{2}} .
$$

This gives the $\tilde{2} \tilde{2}$ component of Eqs. (77) and (B17) and completes the demonstration of this result.

It remains to verify Eq. (78), the relation between slashed objects and the infinite-volume matrix, $\mathcal{K}$. In the main text we derived the relations for $\mathbf{K}_{L, \tilde{2} 3}$ and $\mathbf{K}_{L, 33}$, Eqs. (75) and (76), respectively. We find the result for $\mathbf{K}_{L, 32}$ is essentially the reflection of that for $\mathbf{K}_{L, \tilde{2} 3}$,

$$
\mathbf{K}_{L, 3 \tilde{2}}=\mathbf{K}_{3 \tilde{2}} \frac{1}{1-\mathbf{F}_{\rho \pi} \mathbf{K}_{\tilde{2} \tilde{2}}} .
$$

To complete the discussion we must address $\mathbf{K}_{L, \tilde{2} \tilde{2}}$. Following the same decomposition strategy one last time we reach

$$
\mathbf{K}_{L, \tilde{2} \tilde{2}}=\mathbf{K}_{\tilde{2} \tilde{2}} \mathbf{F}_{\rho \pi} \mathbf{K}_{L, \tilde{2} \tilde{2}}+\mathbf{K}_{\tilde{2} \tilde{2}},
$$

whose solution is

$$
\mathbf{K}_{L, \tilde{2} \tilde{2}}=\frac{1}{1-\mathbf{K}_{\tilde{2} \tilde{2}} \mathbf{F}_{\rho \pi}} \mathbf{K}_{\tilde{2} \tilde{2}}
$$

Our claim is that the four results (75), (76), (B22) and (B24) are equivalent to the matrix result, Eq. (78). To show this, we rearrange the latter, and insert the definitions for $\mathcal{K}$ and $\mathcal{F}_{\rho \pi}$, yielding

$$
\begin{aligned}
\mathbf{K}_{L}= & {\left[\left(\begin{array}{ll}
1 & 0 \\
0 & 1
\end{array}\right)-\left(\begin{array}{ll}
\mathbf{K}_{\tilde{2} \tilde{2}} & \mathbf{K}_{\tilde{2} 3} \\
\mathbf{K}_{3 \tilde{2}} & \mathbf{K}_{33}
\end{array}\right)\left(\begin{array}{cc}
\mathbf{F}_{\rho \pi} & 0 \\
0 & 0
\end{array}\right)\right]^{-1} } \\
& \times\left(\begin{array}{ll}
\mathbf{K}_{\tilde{2} \tilde{2}} & \mathbf{K}_{\tilde{2} 3} \\
\mathbf{K}_{3 \tilde{2}} & \mathbf{K}_{33}
\end{array}\right) .
\end{aligned}
$$

It is then straightforward to pick out various components of the equation by expanding the squarebracketed quantity, identifying a given component and then resumming. The manipulations are simplified by the fact that the matrix containing $\mathbf{F}_{\rho \pi}$ is a projector. The most complicated example is the 33 component, for which we find 
(a)
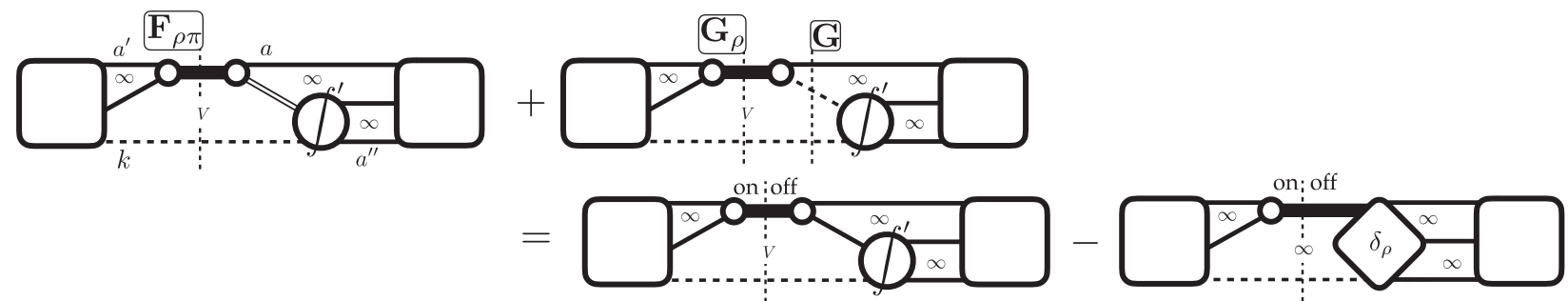

(b)
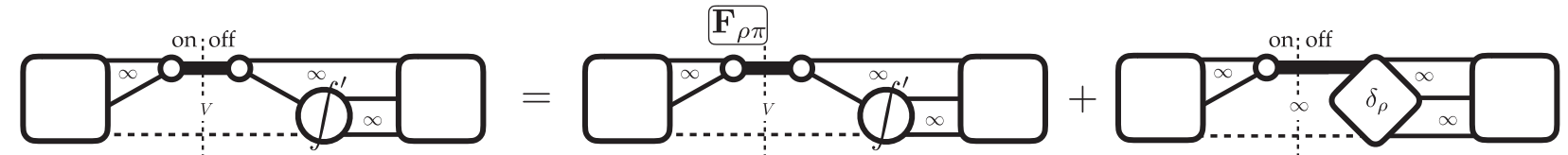

(c)
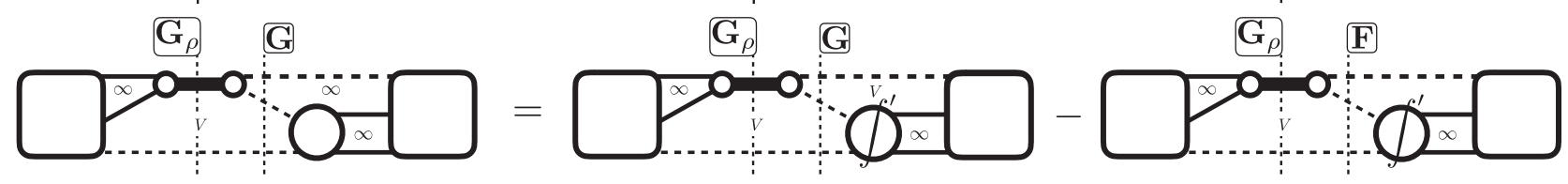

FIG. 8. Derivation of Eq. (B31), using the notation of Fig. 7.

$$
\mathbf{K}_{L, 33}=\mathbf{K}_{33}+\mathbf{K}_{3 \tilde{2}} \sum_{n=0}^{\infty} \mathbf{F}_{\rho \pi}\left(\mathbf{K}_{\tilde{2} \tilde{2}} \mathbf{F}_{\rho \pi}\right)^{n} \mathbf{K}_{\tilde{2} 3},
$$

which sums into Eq. (76). Similarly one can show that the 23 component of the matrix relation matches Eq. (75), the $3 \tilde{2}$ component yields Eq. (B22), and the $\tilde{2} \tilde{2}$ component gives Eq. (B24).

At this stage we have derived all relations summarized in Eqs. (77) and (78) of Sec. III E. From this point the discussion in the main text completes the derivation, yielding a decomposition of all entries the matrix $\mathbf{K}_{L}^{(u)}$ in terms of infinite-volume divergence-free $\mathrm{K}$ matrices.

\section{Volume independence of $\delta C_{\infty}^{\left[B_{2}\right],\{0\}}$}

In this Appendix we explain why $\delta C_{\infty}^{\left[B_{2}\right],\{0\}}$, defined in Eq. (94) of the main text, has only exponentially suppressed volume dependence and can thus be taken as an infinitevolume quantity.

To show this we begin by focusing on the first four terms, and noting that these can be rewritten as

$$
\begin{gathered}
-\frac{2}{3} \boldsymbol{\sigma}^{*} \mathbf{F} \boldsymbol{\sigma}^{\dagger *}-2 \mathbf{A}_{3}^{\prime(s)} \mathbf{F} \boldsymbol{\sigma}^{\dagger *}-\mathbf{A}_{3}^{\prime} \mathbf{F} 2 \mathbf{A}_{3}^{(s)}+\frac{2}{3} \mathbf{A}_{3}^{\prime} \mathbf{F} \mathbf{A}_{3} \\
=-\frac{2}{3} \mathbf{A}_{3}^{\prime(s-u)} \mathbf{F} \boldsymbol{\sigma}^{*}-\frac{2}{3} \mathbf{A}_{3}^{\prime} \mathbf{F} \mathbf{A}_{3}^{(s-u)}
\end{gathered}
$$

where $^{22}$

$$
\begin{aligned}
& \mathbf{A}_{3}^{\prime(s-u)} \equiv \mathbf{A}_{3}^{\prime(s)}-\mathbf{A}_{3}^{\prime(u)}+\boldsymbol{\sigma}^{*}, \quad \text { and } \\
& \mathbf{A}_{3}^{(s-u)} \equiv \mathbf{A}_{3}^{(s)}-\mathbf{A}_{3}^{(u)}+\boldsymbol{\sigma}^{\dagger *} .
\end{aligned}
$$

\footnotetext{
${ }^{22}$ The factors of $\boldsymbol{\sigma}^{*}$ and $\boldsymbol{\sigma}^{\dagger *}$ appear here because $\mathbf{A}_{3}^{\prime(u)}$ and $\mathbf{A}_{3}^{(u)}$ are defined to include the $n=0$ terms [see Eq. (53)] while $\mathbf{A}_{3}^{\prime(s)}$ and $\mathbf{A}_{3}^{(s)}$ do not [see Eq. (57)].
}

As explained in Ref. [5] [see Eqs. (196)-(198) of that work, and the surrounding discussion], the $s-u$ differences in Eq. (B27) can be written as

$$
\begin{aligned}
&- \frac{2}{3} \mathbf{A}_{3}^{\prime(s-u)} \mathbf{F} \boldsymbol{\sigma}^{*}-\frac{2}{3} \mathbf{A}_{3}^{\prime} \mathbf{F} \mathbf{A}_{3}^{(s-u)} \\
&=-\mathbf{A}_{3}^{\prime(s-u)} \frac{i \rho}{3 \omega} \boldsymbol{\sigma}^{*}-\mathbf{A}_{3}^{\prime} \frac{i \rho}{3 \omega} \mathbf{A}_{3}^{(s-u)}+\mathcal{O}\left(e^{-m L}\right) .
\end{aligned}
$$

The phase-space factor $\rho$ [defined in Eq. (27)] is smooth, allowing the implicit sums in our matrix notation to be replaced by integrals, up to exponentially suppressed corrections. Thus, within the framework of dropping exponentially suppressed volume dependence, the righthand side is an infinite-volume quantity.

The remaining two terms in Eq. (94) are

$$
\mathbf{A}_{\tilde{2}}^{\prime} \mathbf{F}_{\rho \pi}\left(\mathbf{A}_{\tilde{2}}-\mathbf{A}_{\tilde{2}}\right)+\mathbf{A}_{\tilde{2}}^{\prime} \mathbf{G}_{\rho} \boldsymbol{\Gamma}\left[\mathbf{G}\left(\mathbf{A}_{3}^{(u)}-\boldsymbol{\sigma}^{\dagger *}\right)-\mathbf{F} 2 \mathbf{A}_{3}^{(s)}\right] .
$$

To show that this is also an infinite-volume quantity, we need a new argument, since this quantity involves K-matrix poles and thus was not encountered in Ref. [5]. We make the argument diagrammatically in Fig. 8, based in part on the derivation illustrated in Fig. 7. We do not give the corresponding analytic expressions, as our earlier discussion explains the precise relation between diagrams and equations.

We begin by substituting Fig. 7(d) into the final term in Fig. $7(\mathrm{c})$ and rearranging so that the terms involving $\mathbf{F}_{\rho \pi}$ and $\mathbf{G}_{\rho}$ are on the left-hand side. We also make several changes to the parts of the diagrams away from the cuts in order to apply the result to the present quantities of interest. These changes do not impact the derivation. They are (a) the box on the left end now represents $\mathbf{A}_{\tilde{2}}^{\prime}$, with the final loop explicitly exposed; (b) the loops to the right of 
the cut are changed from sums to integrals; (c) the kernel on the right is changed from $\mathcal{K}_{2}$ to $\tilde{\mathcal{K}}_{2}^{\prime}$; and, finally, (d), the box on the right end represents the remainder of a full infinite-volume end cap. These steps lead to the equality in Fig. 8(a). At this stage, the first term on the left-hand side represents $\mathbf{A}_{\tilde{2}}^{\prime} \mathbf{F}_{\rho \pi} \mathbf{A}_{\tilde{2}}$, the second term on the right-hand side is manifestly an infinite-volume quantity, while the other two terms need further manipulations to bring them to a useful form.

The first term on the right-hand side of Fig. 8(a) is rewritten in Fig. 8(b). The approach here is to expand the off-shell $\mathbf{A}_{\tilde{2}}$ factor lying to the right of the cut about the position of the K-matrix pole, using the $\delta_{\rho}$ operator introduced above. The leading term gives $\mathbf{A}_{\tilde{2}}^{\prime} \mathbf{F}_{\rho \pi} \mathbf{A}_{\tilde{2}}$, while the $\delta_{\rho}$-dependent term is smooth at the $\mathrm{K}$-matrix pole allowing the sum over the spectator momentum to be replaced by an integral (up to exponentially suppressed corrections). This is shown in the second term on the right-hand side by the " $\infty$ " symbol within the vertical dashed line. This term is manifestly an infinite-volume quantity.

The final step is shown in Fig. 8(c), where we derive an equality for the second term on the right-hand side of Fig. 8(a). On the left-hand side we have a $\mathbf{G}$ cut with the momentum $\vec{a}$ integrated. To obtain the right-hand side we replace this integral with a sum minus a sum-integral difference. The sum gives $\mathbf{A}_{\tilde{2}}^{\prime} \mathbf{G}_{\rho} \boldsymbol{\Gamma} \mathbf{G} \mathbf{A}_{3}^{(u)}$, shown by the first term on the right-hand side of Fig. 8(c). The sumintegral difference gives rise to a factor of $\mathbf{F}$, and, following the arguments of Ref. [5], switches $\mathbf{A}_{3}^{(u)}-\boldsymbol{\sigma}^{\dagger *}$ to $\mathbf{A}_{3}^{(s)}$, leading to $-\mathbf{A}_{\tilde{2}}^{\prime} \mathbf{G}_{\rho} \boldsymbol{\Gamma} \mathbf{F} 2 \mathbf{A}_{3}^{(s)}$.

The overall result of these steps is ${ }^{23}$

$$
\begin{gathered}
\mathbf{A}_{\tilde{2}}^{\prime} \mathbf{F}_{\rho \pi} \mathbf{A}_{\tilde{2}}+\mathbf{A}_{\tilde{2}}^{\prime} \mathbf{G}_{\rho} \boldsymbol{\Gamma}\left[\mathbf{G}\left(\mathbf{A}_{3}^{(u)}-\boldsymbol{\sigma}^{\dagger *}\right)-\mathbf{F} 2 \mathbf{A}_{3}^{(s)}\right] \\
=\mathbf{A}_{\tilde{2}}^{\prime} \mathbf{F}_{\rho \pi} \mathbf{A}_{\tilde{2}}+\delta^{\prime} C_{\infty}^{\left[B_{2}\right],\{0\}}+\mathcal{O}\left(e^{-m L}\right),
\end{gathered}
$$

where $\delta^{\prime} C_{\infty}^{\left[B_{2}\right],\{0\}}$ is a particular infinite-volume contribution, to be absorbed into $\delta C_{\infty}^{\left[B_{2}\right],\{0\}}$ and ultimately into $C_{\infty}^{\left[B_{2}\right]}$. After rearrangement, this demonstrates the desired result.

\section{Symmetrization of factors adjacent to $(X+Y)_{33}$}

In this final Appendix, we demonstrate that the contribution of the 33 component of $\mathbf{X}+\mathbf{Y}$ to $\mathbf{K}_{\mathrm{df}}^{(u)}(\mathbf{X}+\mathbf{Y}) \mathbf{K}_{\mathrm{df}}^{(u)}$ is consistent with the claimed general result, Eq. (149). For definiteness, we consider the term containing the 23 component of $\mathbf{K}_{\mathrm{df}}^{(u)}$ and the 32 component of $\mathbf{K}_{\mathrm{df}}^{(u)}$, although the derivation works for any $(u)$-type threeparticle quantities on the ends. To match with Eq. (149) we need to show that

$$
\begin{aligned}
\chi \equiv & \mathbf{K}_{\mathrm{df}, \tilde{2} 3}^{(u)}(\mathbf{X}+\mathbf{Y})_{33} \mathbf{K}_{\mathrm{df}, 3 \tilde{2}}^{(u)} \\
= & \mathbf{K}_{\mathrm{df}, \tilde{2} 3}^{(u)} \frac{1}{1-\otimes \mathcal{I}_{33}^{\dagger}}\left(\otimes \mathcal{I}_{3 \tilde{2}} \quad \mathcal{S}\right) \cdot\left(\begin{array}{ll}
\mathbf{F}_{\tilde{2} \tilde{2}} & \mathbf{F}_{\tilde{2} 3} \\
\mathbf{F}_{3 \tilde{2}} & \mathbf{F}_{33}
\end{array}\right) \\
& \cdot\left(\begin{array}{c}
\mathcal{I}_{\tilde{2} 3} \otimes \\
\mathcal{S}
\end{array}\right) \frac{1}{1-\mathcal{I}_{33} \otimes} \mathbf{K}_{\mathrm{df}, 3 \tilde{2}}^{(u)}+\delta^{\prime} \mathbf{K}_{\mathrm{df}, \tilde{2} \tilde{2}}^{(u)},
\end{aligned}
$$

where $\delta^{\prime} \mathbf{K}_{\mathrm{df}, \tilde{2} \tilde{2}}^{(u)}$ is an infinite-volume quantity that can be absorbed into the quantity $\delta \mathbf{K}_{\mathrm{df}, \tilde{2} \tilde{2}}^{(u)}$ defined in Eq. (149). Its explicit expression is determined below.

Using the definition of $\mathbf{X}+\mathbf{Y}$ in Eq. (117) we find

$$
\chi=\mathbf{K}_{\mathrm{df}, \tilde{2} 3}^{(u)}(\mathbf{F}+\mathbf{G}) \frac{1}{1-\mathbf{K}_{2}(\mathbf{F}+\mathbf{G})} \mathbf{K}_{\mathrm{df}, 3 \tilde{2}}^{(u)},
$$

which can be divided into two contributions using Eq. (127),

$$
\chi=\chi_{a}+\chi_{b},
$$

$\chi_{a}$ can be rewritten using the reflected form of Eq. (127),

$$
\begin{gathered}
\chi_{a}=\chi_{a 1}+\chi_{a 2}+\chi_{a 3}, \\
\chi_{a 1}=\mathbf{K}_{\mathrm{df}, \tilde{2} 3}^{(u)} \frac{1}{1-\otimes \mathcal{I}_{33}^{\dagger}}\left\{\otimes \mathcal{I}_{32} \mathbf{G}_{\rho} \boldsymbol{\Gamma}+\mathcal{S} \mathbf{F} \mathbf{K}_{2}\right\} \frac{1}{1-(\mathbf{F}+\mathbf{G}) \mathbf{K}_{2}}(\mathbf{F}+\mathbf{G})\left\{\mathbf{K}_{2} \mathbf{F} \mathcal{S}+\boldsymbol{\Gamma} \overline{\mathbf{G}}_{\rho} \mathcal{I}_{\tilde{2} 3} \otimes\right\} \frac{1}{1-\mathcal{I}_{33} \otimes} \mathbf{K}_{\mathrm{df}, 3 \tilde{2}}^{(u)},
\end{gathered}
$$




$$
\begin{gathered}
\chi_{a 2}=\mathbf{K}_{\mathrm{df}, \tilde{2} 3}^{(u)} \frac{1}{1-\otimes \mathcal{I}_{33}^{\dagger}}(\mathbf{F}+\mathbf{G}) \mathbf{K}_{2} \mathbf{F} \mathcal{S} \frac{1}{1-\mathcal{I}_{33} \otimes} \mathbf{K}_{\mathrm{df}, 3 \tilde{2}}^{(u)}, \\
\chi_{a 3}=\mathbf{K}_{\mathrm{df}, \tilde{2} 3}^{(u)} \frac{1}{1-\otimes \mathcal{I}_{33}^{\dagger}}(\mathbf{F}+\mathbf{G}) \mathbf{\Gamma} \overline{\mathbf{G}}_{\rho} \mathcal{I}_{\tilde{2} 3} \otimes \frac{1}{1-\mathcal{I}_{33} \otimes} \mathbf{K}_{\mathrm{df}, 3 \tilde{2} \tilde{}}^{(u)} .
\end{gathered}
$$

$\chi_{a 1}$ includes only symmetrized quantities, but $\chi_{a 2}$ and $\chi_{a 3}$ need further work. Noting the presence of $\mathbf{F}+\mathbf{G}$, we can apply Eq. (126) to both quantities. For $\chi_{a}$ this leads to

$$
\begin{gathered}
\chi_{a 2}=\chi_{a 2 A}+\chi_{a 2 B}, \\
\chi_{a 2 A}=\mathbf{K}_{\mathrm{df}, \tilde{2} 3}^{(u)} \frac{1}{1-\otimes \mathcal{I}_{33}^{\dagger}} \mathcal{S} \mathbf{F} \mathbf{K}_{2} \mathbf{F} \mathcal{S} \frac{1}{1-\mathcal{I}_{33} \otimes} \mathbf{K}_{\mathrm{df}, 3 \tilde{2}}^{(u)}+\mathbf{K}_{\mathrm{df}, \tilde{2} 3}^{(u)} \frac{1}{1-\otimes \mathcal{I}_{33}^{\dagger}} \otimes \mathcal{I}_{3 \tilde{2}} \mathbf{G}_{\rho} \Gamma \mathbf{F} \mathcal{S} \frac{1}{1-\mathcal{I}_{33} \otimes} \mathbf{K}_{\mathrm{df}, 3 \tilde{2}}^{(u)}, \\
\chi_{a 2 B}=\mathbf{K}_{\mathrm{df}, \tilde{2} 3}^{(u)} \frac{1}{1-\otimes \mathcal{I}_{33}^{\dagger}} \otimes \mathcal{I}_{33}^{\dagger} \mathbf{F} \mathcal{S} \frac{1}{1-\mathcal{I}_{33} \otimes} \mathbf{K}_{\mathrm{df}, 3 \tilde{2} \tilde{c}}^{(u)} .
\end{gathered}
$$

Only $\chi_{a 2 B}$ contains an unsymmetrized quantity. It can be rewritten as

$\chi_{a 2 B}=\frac{1}{3} \mathbf{K}_{\mathrm{df}, \tilde{2} 3}^{(u)} \frac{1}{1-\otimes \mathcal{I}_{33}^{\dagger}} \otimes \mathcal{I}_{33}^{\dagger} \mathcal{S} \mathbf{F} \mathcal{S} \frac{1}{1-\mathcal{I}_{33} \otimes} \mathbf{K}_{\mathrm{df}, 3 \tilde{2}}^{(u)}+\left(\mathbf{K}_{\mathrm{df}, \tilde{2} 3}^{(u)} \frac{1}{1-\otimes \mathcal{I}_{33}^{\dagger}} \otimes \mathcal{I}_{33}^{\dagger}\right)^{(u-s)} \frac{i \rho}{3 \omega} \mathcal{S} \frac{1}{1-\mathcal{I}_{33} \otimes} \mathbf{K}_{\mathrm{df}, 3 \tilde{2}}^{(u)}$,

where the first term is symmetrized, while in the second the two factors of $\mathbf{K}_{\mathrm{df}}^{(u)}$ are bound together by an integral operator, giving a contribution to $\delta^{\prime} \mathbf{K}_{\mathrm{df}, \tilde{2} \tilde{2}}^{(u)}$.

Returning to $\chi_{a 3}$, we can apply the reflected form of Eq. (135), yielding

$$
\begin{aligned}
\chi_{a 3}= & \mathbf{K}_{\mathrm{df}, \tilde{2} 3}^{(u)} \frac{1}{1-\otimes \mathcal{I}_{33}^{\dagger}} \mathcal{S} \mathbf{F} \Gamma \overline{\mathbf{G}}_{\rho} \mathcal{I}_{\tilde{2} 3} \otimes \frac{1}{1-\mathcal{I}_{33} \otimes} \mathbf{K}_{\mathrm{df}, 3 \tilde{2}}^{(u)}+\mathbf{K}_{\mathrm{df}, \tilde{2} 3}^{(u)} \frac{1}{1-\otimes \mathcal{I}_{33}^{\dagger}} \otimes \mathcal{I}_{3 \tilde{2}} \mathbf{F}_{\rho \pi} \mathcal{I}_{\tilde{2} 3} \otimes \frac{1}{1-\mathcal{I}_{33} \otimes} \mathbf{K}_{\mathrm{df}, 3 \tilde{2}}^{(u)} \\
& +\mathbf{K}_{\mathrm{df}, \tilde{2} 3}^{(u)} \frac{1}{1-\otimes \mathcal{I}_{33}^{\dagger}} \otimes \rho_{3 \tilde{2}} \otimes \mathcal{I}_{\tilde{2} 3} \otimes \frac{1}{1-\mathcal{I}_{33} \otimes} \mathbf{K}_{\mathrm{df}, \tilde{2} \tilde{2}}^{(u)} .
\end{aligned}
$$

The final term in this expression gives an additional contribution to $\delta^{\prime} \mathbf{K}_{\mathrm{df}, \tilde{2} \tilde{2}}^{(u)}$.

The final step is to analyze $\chi_{b}$. This requires the result

$$
\mathbf{K}_{\mathrm{df}, \tilde{2} 3}^{(u)} \mathbf{G} \mathbf{K}_{\mathrm{df}, 3 \tilde{2}}^{(u)}=\mathbf{K}_{\mathrm{df}, \tilde{2} 3}^{(u)} \mathbf{F} \mathbf{K}_{\mathrm{df}, 3 \tilde{2}}^{(s+\tilde{2})}+\mathbf{K}_{\mathrm{df}, \tilde{2} 3}^{(u)} \otimes \rho_{33} \otimes \mathbf{K}_{\mathrm{df}, 3 \tilde{2}}^{(u)},
$$

which implies

$$
\begin{aligned}
\mathbf{K}_{\mathrm{df}, \tilde{2} 3}^{(u)}(\mathbf{F}+\mathbf{G}) \mathbf{K}_{\mathrm{df}, 3 \tilde{2}}^{(u)} & =\mathbf{K}_{\mathrm{df}, \tilde{2} 3}^{(u)} \mathbf{F} \mathcal{S} \mathbf{K}_{\mathrm{df}, 3 \tilde{2}}^{(u)}+\mathbf{K}_{\mathrm{df}, \tilde{2} 3}^{(u)} \otimes \rho_{33} \otimes \mathbf{K}_{\mathrm{df}, \tilde{2} \tilde{2}}^{(u)}, \\
& =\frac{1}{3} \mathbf{K}_{\mathrm{df}, \tilde{2} 3}^{(u)} \mathcal{S} \mathbf{F} \mathcal{S} \mathbf{K}_{\mathrm{df}, 3 \tilde{2}}^{(u)}+\mathbf{K}_{\mathrm{df}, \tilde{2} 3}^{(u)} \otimes \rho_{33} \otimes \mathbf{K}_{\mathrm{df}, 3 \tilde{2}}^{(u)}+\mathbf{K}_{\mathrm{df}, \tilde{2} 3}^{(u-s)} \frac{i \rho}{3 \omega} \mathbf{K}_{\mathrm{df}, 3 \tilde{2} \tilde{}}^{(u)} .
\end{aligned}
$$

The derivation of Eq. (B47) is shown diagrammatically in Fig. 9. The result holds, as usual, for any choice of unsymmetrized $(u)$-like three-particle quantities on the ends, and thus can be applied to $\chi_{b}$, yielding

$$
\chi_{b}=\frac{1}{3} \mathbf{K}_{\mathrm{df}, \tilde{2} 3}^{(u)} \mathcal{S} \mathbf{F} \mathcal{S} \frac{1}{1-\mathcal{I}_{33} \otimes} \mathbf{K}_{\mathrm{df}, 3 \tilde{2}}^{(u)}+\mathbf{K}_{\mathrm{df}, \tilde{2} 3}^{(u)} \otimes \rho_{33} \otimes \frac{1}{1-\mathcal{I}_{33} \otimes} \mathbf{K}_{\mathrm{df}, 3 \tilde{2}}^{(u)}+\mathbf{K}_{\mathrm{df}, \tilde{2} 3}^{(u-s} \frac{i \rho}{3 \omega} \frac{1}{1-\mathcal{I}_{33} \otimes} \mathbf{K}_{\mathrm{df}, 3 \tilde{2}}^{(u)}
$$

The last two terms give additional contributions to $\delta^{\prime} \mathbf{K}_{\mathrm{df}, \tilde{2} \tilde{2}}^{(u)}$. 


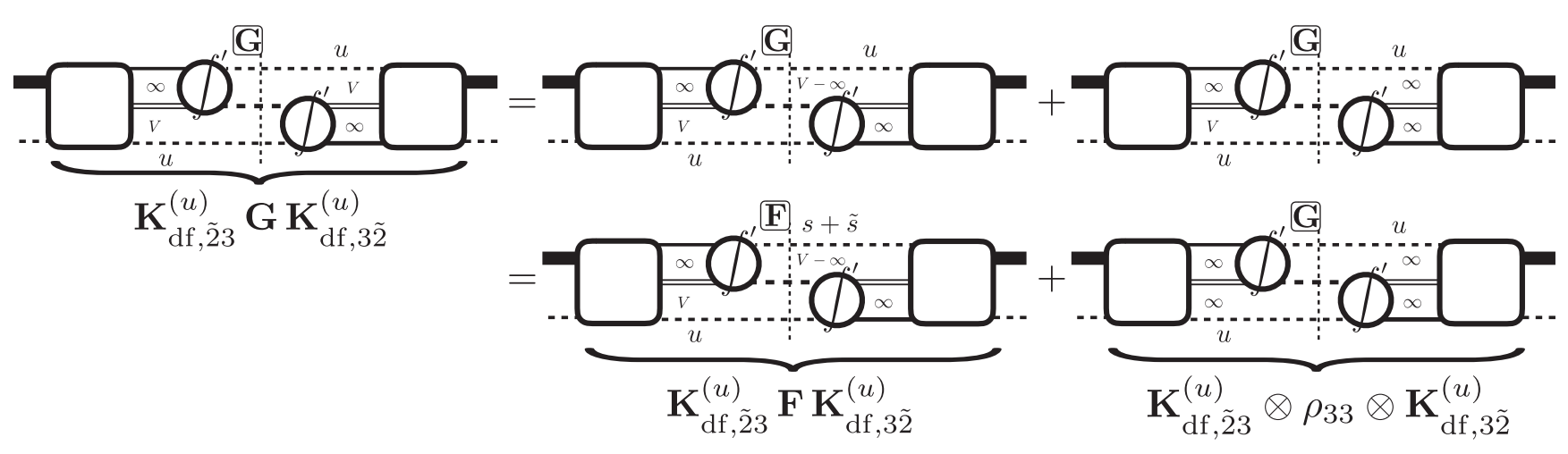

FIG. 9. Derivation of Eq. (B47), using the notation of Figs. 1, 4 and 7. The first step is identical to that in Fig. 4: replacing the sum adjacent to the $G$ cut with a sum-integral difference and an integral, the former giving rise to an $F$ cut. In this case, however, there are no K-matrix poles, so the integral in the upper-right loop of the final term on the first line removes the divergence in the summand for the spectator momentum, allowing it to be replaced by an integral, as shown by the final term on the second line. Since all loops are integrated in this term, it can be represented as the action of a new integral operator, denoted $\rho_{33}$, tying the $\mathbf{K}_{\mathrm{df}, \tilde{2} 3}^{(u)}$ and $\mathbf{K}_{\mathrm{df}, 3 \tilde{2}}^{(u)}$ together.

Combining all these results we find the desired result, Eq. (B33), with

$$
\begin{aligned}
\delta^{\prime} \mathbf{K}_{\mathrm{df}, \tilde{2} \tilde{2}}^{(u)}= & \left(\mathbf{K}_{\mathrm{df}, \tilde{2} 3}^{(u)} \frac{1}{1-\otimes \mathcal{I}_{33}^{\dagger}} \otimes \mathcal{I}_{33}^{\dagger}\right)^{(u-s)} \frac{i \rho}{3 \omega} \mathcal{S}_{\frac{1}{1-\mathcal{I}_{33} \otimes}} \mathbf{K}_{\mathrm{df}, 3 \tilde{2}}^{(u)}+\mathbf{K}_{\mathrm{df}, \tilde{2} 3}^{(u)} \frac{1}{1-\otimes \mathcal{I}_{33}^{\dagger}} \otimes \rho_{3 \tilde{2}} \otimes \mathcal{I}_{\tilde{2} 3} \otimes \frac{1}{1-\mathcal{I}_{33} \otimes} \mathbf{K}_{\mathrm{df}, 3 \tilde{2}}^{(u)} \\
& +\mathbf{K}_{\mathrm{df}, \tilde{2} 3}^{(u)} \otimes \rho_{33} \mathbf{K}_{\mathrm{df}, 3 \tilde{2}}^{(u)}+\mathbf{K}_{\mathrm{df}, \tilde{2} 3}^{(u-s} \frac{i \rho}{3 \omega} \mathbf{K}_{\mathrm{df}, 3 \tilde{2}}^{(u)} .
\end{aligned}
$$

[1] D. J. Wilson, Proc. Sci., LATTICE2016 (2016) 016.

[2] R. A. Briceño, J. J. Dudek, and R. D. Young, Rev. Mod. Phys. 90, 025001 (2018).

[3] D. Mohler, EPJ Web Conf. 181, 01027 (2018).

[4] G. K. C. Cheung, C. E. Thomas, J. J. Dudek, and R. G. Edwards (Hadron Spectrum Collaboration), J. High Energy Phys. 11 (2017) 033.

[5] M. T. Hansen and S. R. Sharpe, Phys. Rev. D 90, 116003 (2014).

[6] M. T. Hansen and S. R. Sharpe, Phys. Rev. D 92, 114509 (2015).

[7] R. A. Briceño, M. T. Hansen, and S. R. Sharpe, Phys. Rev. D 95, 074510 (2017).

[8] K. Polejaeva and A. Rusetsky, Eur. Phys. J. A 48, 67 (2012).

[9] R. A. Briceño and Z. Davoudi, Phys. Rev. D 87, 094507 (2013).

[10] S. Aoki, N. Ishii, T. Doi, Y. Ikeda, and T. Inoue, Phys. Rev. D 88, 014036 (2013).

[11] H.-W. Hammer, J.-Y. Pang, and A. Rusetsky, J. High Energy Phys. 09 (2017) 109.

[12] H. W. Hammer, J. Y. Pang, and A. Rusetsky, J. High Energy Phys. 10 (2017) 115.

[13] P. Guo and V. Gasparian, Phys. Lett. B 774, 441 (2017).

[14] M. Mai and M. Döring, Eur. Phys. J. A 53, 240 (2017).
[15] M. Döring, H. W. Hammer, M. Mai, J. Y. Pang, A. Rusetsky, and J. Wu, Phys. Rev. D 97, 114508 (2018).

[16] R. A. Briceño, M. T. Hansen, and S. R. Sharpe, Phys. Rev. D 98, 014506 (2018).

[17] M. Mai and M. Döring, arXiv:1807.04746.

[18] M. Lüscher, Commun. Math. Phys. 105, 153 (1986).

[19] M. Lüscher, Nucl. Phys. B354, 531 (1991).

[20] K. Rummukainen and S. A. Gottlieb, Nucl. Phys. B450, 397 (1995).

[21] C. h. Kim, C. T. Sachrajda, and S. R. Sharpe, Nucl. Phys. B727, 218 (2005).

[22] S. He, X. Feng, and C. Liu, J. High Energy Phys. 07 (2005) 011.

[23] M. T. Hansen and S. R. Sharpe, Phys. Rev. D 86, 016007 (2012).

[24] R. A. Briceño and Z. Davoudi, Phys. Rev. D 88, 094507 (2013).

[25] R. A. Briceño, Phys. Rev. D 89, 074507 (2014).

[26] J. J. Dudek, R. G. Edwards, C. E. Thomas, and D. J. Wilson (Hadron Spectrum Collaboration), Phys. Rev. Lett. 113, 182001 (2014).

[27] R. A. Briceño, J. J. Dudek, R. G. Edwards, and D. J. Wilson, Phys. Rev. D 97, 054513 (2018).

[28] G. Moir, M. Peardon, S. M. Ryan, C. E. Thomas, and D. J. Wilson, J. High Energy Phys. 10 (2016) 011. 
[29] J. J. Dudek, R. G. Edwards, and D. J. Wilson (Hadron Spectrum Collaboration), Phys. Rev. D 93, 094506 (2016).

[30] D. J. Wilson, R. A. Briceño, J. J. Dudek, R. G. Edwards, and C. E. Thomas, Phys. Rev. D 92, 094502 (2015).

[31] A. Woss, C. E. Thomas, J. J. Dudek, R. G. Edwards, and D. J. Wilson, J. High Energy Phys. 07 (2018) 043.

[32] See Supplemental Material at http://link.aps.org/ supplemental/10.1103/PhysRevD.99.014516, for a Mathematica notebook based on the package 'NCAlgebra'. Here we check various identities used in the derivation, all involving matrices of undetermined size treated as generic non-commuting objects.
[33] M. C. de Oliveira, J. W. H. Helton, M. Stankus, and B. Miller, NCAlgebra, Version 5.0.4 (2018), https://github .com/NCAlgebra.

[34] M. Mai, B. Hu, M. Döring, A. Pilloni, and A. Szczepaniak, Eur. Phys. J. A 53, 177 (2017).

[35] A. Jackura, C. Fernandez-Ramirez, V. Mathieu, M. Mikhasenko, J. Nys, A. Pilloni, K. Saldana, N. Sherrill, and A. P. Szczepaniak, arXiv:1809.10523.

[36] R. A. Briceño and M. T. Hansen, Phys. Rev. D 94, 013008 (2016). 